\title{
Anatomy of $\varepsilon^{\prime} / \varepsilon$ beyond the standard model
}

\author{
Jason Aebischer $^{1, \mathrm{a}}$, Christoph Bobeth ${ }^{1,2, \mathrm{~b}}$, Andrzej J. Buras ${ }^{3, \mathrm{c}}$, David M. Straub ${ }^{1, \mathrm{~d}}$ \\ ${ }^{1}$ Excellence Cluster Universe, Boltzmannstr. 2, 85748 Garching, Germany \\ 2 Physik Department, TU München, James-Franck-Straße, 85748 Garching, Germany \\ ${ }^{3}$ TUM Institute for Advanced Study, Lichtenbergstr. 2a, 85748 Garching, Germany
}

Received: 14 November 2018 / Accepted: 22 February 2019 / Published online: 11 March 2019

(C) The Author(s) 2019

\begin{abstract}
We present for the first time a model-independent anatomy of the ratio $\varepsilon^{\prime} / \varepsilon$ in the context of the $\Delta S=1$ effective theory with operators invariant under QCD and QED and in the context of the standard model effective field theory (SMEFT) with the operators invariant under the full SM gauge group. Our goal is to identify the new physics scenarios that are probed by this ratio and which could help to explain a possible deviation from the SM that is hinted by the data. To this end we derive a master formula for $\varepsilon^{\prime} / \varepsilon$, which can be applied to any theory beyond the standard model (BSM) in which the Wilson coefficients of all contributing operators have been calculated at the electroweak scale. The relevant hadronic matrix elements of BSM operators are from the Dual QCD approach and the SM ones from lattice QCD. Within SMEFT, the constraints from $K^{0}$ and $D^{0}$ mixing as well as electric dipole moments limit significantly potential new physics contributions to $\varepsilon^{\prime} / \varepsilon$. Correlations of $\varepsilon^{\prime} / \varepsilon$ with $K \rightarrow \pi \nu \bar{v}$ decays are briefly discussed. Building on our EFT analysis and the model-independent constraints, we discuss implications of a possible deviation from the SM in $\varepsilon^{\prime} / \varepsilon$ for model building, highlighting the role of the new scalar and tensor matrix elements in models with scalar mediators.
\end{abstract}

\section{Contents}

1 Introduction . . . . . . . . . . . 1

2 Model-independent anatomy of $\varepsilon^{\prime} / \varepsilon \ldots \ldots \ldots 3$

$2.1 K \rightarrow \pi \pi$ matrix elements . . . . . . . . 4

2.2 Renormalization group evolution below the electroweak scale . . . . . . . . . 5 5

2.3 Master formula for $\varepsilon^{\prime} / \varepsilon$ beyond the SM . . . 6

\footnotetext{
a e-mail: jason.aebischer@tum.de

be-mail: christoph.bobeth@tum.de

c e-mail: andrzej.buras@tum.de

de-mail: david.straub@tum.de
}

2.4 Matching from SMEFT onto $\Delta S=1 \mathrm{EFT} \ldots .7$

3 Model-independent constraints in SMEFT . . . . 8

$3.1 \Delta S=2 \ldots \ldots \ldots \ldots \ldots$

$3.2 \Delta C=2 \ldots \ldots \ldots \ldots \ldots$

3.3 Interplay of $\Delta S=2$ and $\Delta C=2 \ldots \ldots 10$

3.4 Neutron electric dipole moment . . . . . . 11

$3.5 K \rightarrow \pi v \bar{v}$ and $K \rightarrow \pi \ell^{+} \ell^{-} \ldots \ldots \ldots 12$

$3.6 \Delta C=1 \ldots \ldots \ldots \ldots \ldots \ldots$

4 Implications for model building . . . . . . . . . . 14

4.1 Tree-level mediators . . . . . . . . . . . . 14

4.2 Scalar operators from scalar mediators . . . . . 15

4.3 Models with vector mediators . . . . . . . . . 15

4.4 Models with modified electroweak couplings . 16

4.5 Models with dipole operators . . . . . . . . . 17

5 Summary . . . . . . . . . . . . . . . . . 18

A. $\Delta S=1$ EFT operators . . . . . . . . . 19

B. $K \rightarrow \pi \pi$ matrix elements . . . . . . . . . 20

C. $\varepsilon^{\prime} / \varepsilon$ Master formula for new physics . . . . . . . 20

D. SMEFT operators . . . . . . . . . . . . . . 21

E. SMEFT matching conditions . . . . . . . . . . . 23

E.1 Four-quark operators . . . . . . . . . . 23

E.2 Modified $Z^{0}$ and $W^{ \pm}$couplings . . . . . . . 24

E.3 Dipole operators . . . . . . . . . . . . . . . 25

F. RG evolution in SMEFT . . . . . . . . . . . 26

References . . . . . . . . . . . . . . . 26 26

\section{Introduction}

One of the stars of flavour physics since the early 1980s has been the ratio $\varepsilon^{\prime} / \varepsilon$ that measures the size of direct $\mathrm{CP}$ violation in $K_{L} \rightarrow \pi \pi$ relative to the indirect $\mathrm{CP}$ violation described by $\varepsilon_{K}$. On the experimental side, the world average from the NA48 [1] and $\mathrm{KTeV}$ [2,3] collaborations reads

$\left(\varepsilon^{\prime} / \varepsilon\right)_{\exp }=(16.6 \pm 2.3) \times 10^{-4}$. 
On the theory side, a long-standing challenge in making predictions for $\varepsilon^{\prime} / \varepsilon$ within the standard model (SM) has been the significant cancellation between QCD and electroweak penguin contributions to this ratio. In the SM, QCD penguins give a positive contribution and electroweak penguins a negative one. Therefore, in order to obtain an accurate prediction, both the short-distance contributions to this ratio, represented by Wilson coefficients of penguin operators, as well as the long-distance hadronic matrix elements of these operators have to be accurately known.

As far as the short-distance contributions are concerned, they have been known already for 25 years at next-toleading order (NLO) [4-9]. First steps towards next-to-nextto-leading order (NNLO) predictions for $\varepsilon^{\prime} / \varepsilon$ have been made in [10-13] and further progress towards a complete NNLO result is under way [14].

The situation with hadronic matrix elements is another story and even if significant progress on their evaluation has been made over the last 25 years, the present status is far from satisfactory. The situation of $\varepsilon^{\prime} / \varepsilon$ in the SM can be briefly summarized as follows:

- The analysis of $\varepsilon^{\prime} / \varepsilon$ by the RBC-UKQCD collaboration based on their lattice QCD calculation of $K \rightarrow \pi \pi$ matrix elements $[15,16]$, as well as the analyses performed in $[17,18]$ that are based on the same matrix elements but also include isospin breaking effects, find $\varepsilon^{\prime} / \varepsilon$ in the ballpark of $(1-2) \times 10^{-4}$. This is by one order of magnitude below the data, but with an error in the ballpark of $5 \times 10^{-4}$. Consequently, based on these analyses, one can talk about an $\varepsilon^{\prime} / \varepsilon$ anomaly of at most $3 \sigma$.

- An independent analysis based on hadronic matrix elements from the Dual QCD (DQCD) approach [19,20] gives a strong support to these values and moreover provides an upper bound on $\varepsilon^{\prime} / \varepsilon$ in the ballpark of $6 \times 10^{-4}$.

- A different view has been expressed in [21] where, using ideas from chiral perturbation theory, the authors find $\varepsilon^{\prime} / \varepsilon=(15 \pm 7) \times 10^{-4}$. While in agreement with the measurement, the large uncertainty, that expresses the difficulties in matching long distance and short distance contributions in this framework, does not allow for clear-cut conclusions. Consequently, values above $2 \times 10^{-3}$, that are rather unrealistic from the point of view of lattice QCD and DQCD, are not excluded in this approach.

Here, we would like to point out that all the existing estimates of $\varepsilon^{\prime} / \varepsilon$ at NLO suffer from unaccounted-for shortdistance renormalization scheme uncertainties in the electroweak penguin contributions that are removed in the NNLO matching at the electroweak scale [11]. In the naive dimensional regularization (NDR) scheme, used in all recent analyses, these corrections enhance parts of the electroweak penguin contribution by roughly $16 \%$, thereby leading to a negative shift of $-1.3 \times 10^{-4}$ decreasing the value of $\varepsilon^{\prime} / \varepsilon$, similarly to isospin breaking effects. This could appear small in view of other uncertainties. However, on the one hand, potential scale and renormalization scheme uncertainties have been removed in this manner and on the other hand, one day such corrections could turn out to be relevant. Finally, the fact that this correction further decreases $\varepsilon^{\prime} / \varepsilon$ within the SM gives another motivation for the search for new physics responsible for it, and thus for the present analysis.

Based on the results from RBC-UKQCD and the DQCD approach of 2015 and without the inclusion of NNLO corrections mentioned above, a number of analyses have been performed in specific models beyond the SM (BSM) with the goal to obtain a sufficient upward shift in $\varepsilon^{\prime} / \varepsilon$ and thereby its experimental value. These include in particular tree-level $Z^{\prime}$ exchanges with explicit realization in 331 models [22,23] or models with tree-level $Z^{0}$ exchanges $[24,25]$ with explicit realization in models with mixing of heavy vector-like fermions with ordinary fermions [26] and the Littlest Higgs model with T-parity [27]. Also simplified $Z^{\prime}$ scenarios [28,29], the MSSM [30-34], the type-III Two-Higgs Doublet model (2HDM) [35,36], a $S U(2)_{L} \otimes$ $S U(2)_{R} \otimes U(1)_{B-L}$ model $[37,38]$ and the one based on SU(8) symmetry [39] are of help here. On the other hand, as demonstrated in [40], it is very unlikely that leptoquarks are responsible for the $\varepsilon^{\prime} / \varepsilon$ anomaly when the constraints from rare semi-leptonic and leptonic $K$ decays are taken into account.

An important limitation of the recent literature is that it addressed the $\varepsilon^{\prime} / \varepsilon$ anomaly only in models in which new physics (NP) entered exclusively through modifications of the Wilson coefficients of SM operators. However, generally, BSM operators with different Dirac structures - like the ones resulting from tree-level scalar exchanges and leading to scalar and tensor operators - or chromo-magnetic dipole operators could play a significant role in $\varepsilon^{\prime} / \varepsilon$. Until recently, no quantitative judgment of the importance of such operators was possible because of the absence of even approximate calculations of the relevant hadronic matrix elements in QCD. This situation has been changed through the calculation of the matrix elements in question for the chromo-magnetic dipole operators by lattice QCD [41] and DQCD [42] and in particular through the calculation of matrix elements of all fourquark BSM operators, including scalar and tensor operators, by DQCD [43]. The first application of these new results for chromo-magnetic dipole operators can be found in [36] and in the present paper we will have a closer look at all BSM operators.

Another important question is which of the operators in the low-energy effective theory can be generated in a short-distance BSM scenario. A powerful tool for this pur- 
pose is the standard model effective field theory (SMEFT) $[44,45]$, where the SM Lagrangian above the electroweak scale $\mu_{\mathrm{ew}} \sim 100 \mathrm{GeV}$ and below the scale of new physics $\mu_{\Lambda} \gg \mu_{\text {ew }}$ is supplemented by all dimension five and six operators that are invariant under the SM gauge group $G_{\mathrm{SM}}=S U(3)_{c} \otimes S U(2)_{L} \otimes U(1)_{Y}$. As we will show, matching the SMEFT at tree level on the $\Delta S=1$ effective field theory (EFT) at $\mu_{\mathrm{ew}}$, not all operators that are allowed by the QCD and QED gauge symmetry $S U(3)_{c} \otimes U(1)_{Q}$ are generated.

The goal of the present paper is to perform a general BSM analysis of $\varepsilon^{\prime} / \varepsilon$, taking into account all possible operators and exploiting the SMEFT to single out the operators that can be generated in high-scale BSM scenarios. In this manner, one can obtain a general view on possible BSM physics behind the emerging $\varepsilon^{\prime} / \varepsilon$ anomaly and point out promising directions to be explored in concrete models and exclude those in which the explanation of the data in (1) is unlikely. In the context of SMEFT, constraints from other processes, in particular from $\varepsilon_{K}, D^{0}-\bar{D}^{0}$ mixing, and electric dipole moments, play an important role and we will discuss them in the present paper.

One of the highlights of our paper is the derivation of a master formula for $\varepsilon^{\prime} / \varepsilon$, recently presented in [46], which can be applied to any theory beyond the SM in which the Wilson coefficients of the operators have been calculated at the electroweak scale. The relevant hadronic matrix elements of BSM operators entering this formula are taken from the DQCD approach and for the SM ones from lattice QCD.

The outline of our paper is as follows. In Sect. 2 we present a complete model-independent anatomy of $\varepsilon^{\prime} / \varepsilon$ from the point of view of the $\Delta S=1 \mathrm{EFT}$ and provide the master formula of $\varepsilon^{\prime} / \varepsilon$ beyond the SM. We give also the tree-level matching of SMEFT on the $\Delta S=1$ EFT relevant for $\varepsilon^{\prime} / \varepsilon$. In Sect. 3 we discuss correlations that arise in SMEFT between $\varepsilon^{\prime} / \varepsilon$ and other processes, in particular $\varepsilon_{K}, D^{0}-\bar{D}^{0}$ mixing, the electric dipole moment of the neutron, and the decays $K_{L} \rightarrow \pi^{0} \nu \bar{\nu}$ and $K^{+} \rightarrow \pi^{+} \nu \bar{v}$. Based on the previous section, we derive lessons for model building in Sect. 4 to facilitate the identification of classes of models that are constrained by $\varepsilon^{\prime} / \varepsilon$ as well as singling out prime candidates for new physics scenarios behind the $\varepsilon^{\prime} / \varepsilon$ anomaly. We summarize the main virtues of our analysis in Sect. 5. In several appendices we collect our conventions, recall useful definitions, and provide the necessary material for the numerical analysis of $\varepsilon^{\prime} / \varepsilon$ beyond the SM.

\section{Model-independent anatomy of $\varepsilon^{\prime} / \varepsilon$}

The parameter $\varepsilon^{\prime} / \varepsilon$ measures the ratio of direct over indirect $\mathrm{CP}$ violation in $K_{L} \rightarrow \pi \pi$ decays. Using the precisely mea- sured $\varepsilon_{K}$ from experiment and neglecting isospin breaking corrections, ${ }^{1}$ it can be written as ${ }^{2}$

$\frac{\varepsilon^{\prime}}{\varepsilon}=-\frac{\omega}{\sqrt{2}\left|\varepsilon_{K}\right|}\left[\frac{\operatorname{Im} A_{0}}{\operatorname{Re} A_{0}}-\frac{\operatorname{Im} A_{2}}{\operatorname{Re} A_{2}}\right]$,

where $A_{0,2}$ are the $K \rightarrow \pi \pi$ isospin amplitudes

$A_{0,2}=\left\langle(\pi \pi)_{I=0,2}\left|\mathcal{H}_{\Delta S=1}^{(3)}(\mu)\right| K\right\rangle$,

and the ratio $\omega=\operatorname{Re} A_{2} / \operatorname{Re} A_{0} \approx 1 / 22$ expresses the enhancement of $\operatorname{Re} A_{0}$ over $\operatorname{Re} A_{2}$ known as the $\Delta I=1 / 2$ rule. $\mathcal{H}_{\Delta S=1}^{(3)}$ denotes the effective Hamiltonian of the $\Delta S=1$ EFT taken at the low-energy scale $\mu \sim 1 \mathrm{GeV}$ with only the three lightest quarks, $q=u, d$, $s$ being dynamical. It is obtained by decoupling the heavy $W^{ \pm}, Z^{0}$, and $h^{0}$ bosons and the top quark at the electroweak scale $\mu_{\mathrm{ew}}$ and the bottom and charm quarks at their respective mass thresholds $\mu_{b}$ and $\mu_{c}$, respectively [49].

The values of the Wilson coefficients in this effective Hamiltonian encode all possible NP effects in $\varepsilon^{\prime} / \varepsilon$. However, when considering a NP model at a scale $\mu_{\Lambda}$, much larger than the electroweak scale $\mu_{\text {ew }}$, these low-energy Wilson coefficients are only the final step in a series of effective theories. At $\mu_{\Lambda} \gg \mu_{\mathrm{ew}}$, integrating out the heavy new particles leads to the SMEFT Lagrangian with dimension five and six operators invariant under the full SM gauge group. Using the SMEFT renormalization group (RG) equations, these can be evolved to $\mu_{\mathrm{ew}}$ and matched onto $\mathcal{H}_{\Delta S=1}^{(5)}$ with five active quark flavours. This hierarchy of effective theories is sketched in Fig. 1 and the remainder of this section will be devoted to discussing the individual steps in detail, starting from the lowest scale:

- Section 2.1 discusses the relevant operators in $\mathcal{H}_{\Delta S=1}^{(3)}$ and their $K \rightarrow \pi \pi$ matrix elements.

- Section 2.2 discusses the RG evolution between the lowest scale $\mu$ and $\mu_{\text {ew }}$ and the additional operators in $\mathcal{H}_{\Delta S=1}^{(5)}$ that can play a role.

- Section 2.3 summarizes the results of Sects. 2.1 and 2.2 in the form of a convenient master formula of $\varepsilon^{\prime} / \varepsilon$.

\footnotetext{
1 Isospin breaking corrections have been considered in [47,48] and have been taken into account in the SM analyses in [17,18]. There they play a significant role in suppressing the $\operatorname{Im} A_{0}$ contribution relatively to the $\operatorname{Im} A_{2}$ one, making the cancellation between these two contributions stronger. However, in BSM scenarios, such a strong cancellation is not expected and typically contributions to $\operatorname{Im} A_{2}$ dominate as they are not suppressed by the factor $1 / \omega \approx 22$ in contrast to $\operatorname{Im} A_{0}$. Therefore, the inclusion of isospin breaking effects in the BSM contributions calculated by us is insignificant and it is justified to neglect them in view of the remaining uncertainties in hadronic matrix elements that affect the dominant contributions to $\operatorname{Im} A_{2}$.

2 It is common to omit the subscript $K$ on $\varepsilon \equiv \varepsilon_{K}$ when writing the ratio $\varepsilon^{\prime} / \varepsilon$.
} 


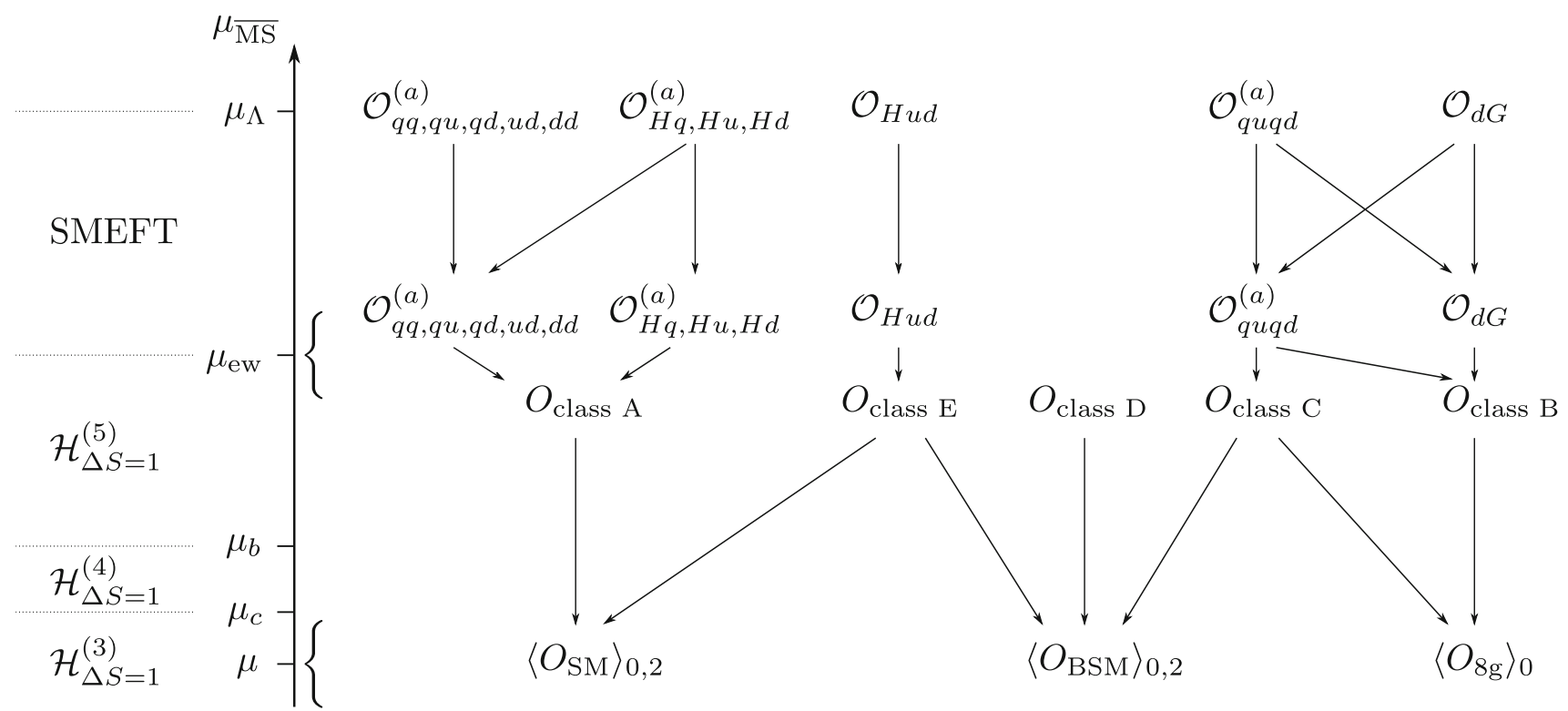

Fig. 1 Sketch of the different contributions to $\varepsilon^{\prime} / \varepsilon$ discussed in Sect. 2, starting from Wilson coefficients of SMEFT operators at a high scale $\mu_{\Lambda}$, evolved to the electroweak scale $\mu_{\text {ew }}$ with the SMEFT RG equations, matched onto the 5-flavour $\Delta S=1$ effective Hamiltonian (Sect. 2.4), evolved to the hadronic scale $\mu$ (Sect. 2.2), and multiplied

- Section 2.4 discusses the matching of SMEFT onto $\mathcal{H}_{\Delta S=1}^{(5)}$ at $\mu_{\mathrm{ew}}$, singling out the operators that arise at the dimension-six level, and briefly discusses RG effects in SMEFT above $\mu_{\mathrm{ew}}$.

Figure 1 can serve as a map guiding through this anatomy and already anticipates some of the findings of this section.

\section{$2.1 K \rightarrow \pi \pi$ matrix elements}

Given the values of the Wilson coefficients in the effective Hamiltonian

$\mathcal{H}_{\Delta S=1}^{(3)}=-\sum_{i} C_{i}(\mu) O_{i}$,

at the low-energy scale $\mu$, the $K \rightarrow \pi \pi$ isospin amplitudes can be calculated by means of (3) if the matrix elements

$\left\langle O_{i}(\mu)\right\rangle_{I} \equiv\left\langle(\pi \pi)_{I}\left|O_{i}\right| K\right\rangle(\mu)$,

are known at the scale $\mu$. Neglecting electro-magnetic corrections, only chromo-magnetic dipole operators

$O_{8 g}^{(\prime)}=m_{s}\left(\bar{s} \sigma^{\mu \nu} T^{A} P_{L(R)} d\right) G_{\mu \nu}^{A}$,

and four-quark operators

$O_{X A B}^{q}=\left(\bar{s}^{i} \Gamma_{X} P_{A} d^{i}\right)\left(\bar{q}^{j} \Gamma_{X} P_{B} q^{j}\right)$, by the $K \rightarrow \pi \pi$ matrix elements (Sect. 2.1). In the SMEFT running, the arrows indicate operator mixing arising from top-quark Yukawa or gauge couplings. The matching is performed at tree level. We have omitted semi-leptonic and electro-magnetic dipole operators

$\widetilde{O}_{X A B}^{q}=\left(\bar{s}^{i} \Gamma_{X} P_{A} d^{j}\right)\left(\bar{q}^{j} \Gamma_{X} P_{B} q^{i}\right)$,

can contribute. Here $i, j$ are colour indices, $A, B=L, R$, and $X=S, V, T$ with $\Gamma_{S}=1, \Gamma_{V}=\gamma^{\mu}, \Gamma_{T}=$ $\sigma^{\mu \nu}{ }^{3}$ Throughout it is sufficient to consider the case $A=$ $L$, whereas results for the chirality-flipped case $A=R$ (obtained by interchange of $L \leftrightarrow R$ for both $A, B$ ) follow analogously due to parity conservation of QCD and QED: the $K \rightarrow \pi \pi$ matrix elements of chirality-flipped operators have just opposite sign.

Since the number of active quark flavours is $N_{f}=3$, in principle the four-quark operators with $q=u, d, s$ are present in (7). However, we expect the contribution to $K \rightarrow$ $\pi \pi$ matrix elements from operators with flavour structure $(\bar{s} d)(\bar{s} s)$ to be strongly suppressed ${ }^{4}$ and we will neglect them.

Using Fierz relations to eliminate redundant operators (see "Appendix A" for details), it then follows that there are only $10+10^{\prime}(\bar{s} d)(\bar{u} u)$ and $5+5^{\prime}(\bar{s} d)(\bar{d} d)$ linearly independent four-quark operators that contribute to $\varepsilon^{\prime} / \varepsilon$ via a non-vanishing $K \rightarrow \pi \pi$ matrix element and in addition the chromo-magnetic dipole operators $\left(1+1^{\prime}\right)$. In the amplitude

\footnotetext{
${ }^{3}$ For $\Gamma_{T}$ there is only $P_{A}=P_{B}$ in four dimensions but not $P_{A} \neq P_{B}$.

4 The $N_{f}=3$ lattice results $[15,16]$ of the $K \rightarrow \pi \pi$ matrix elements in principle include these contributions but from these results they cannot be disentangled from the $(\bar{s} d)(\bar{u} u)$ and $(\bar{s} d)(\bar{d} d)$ ones. In this regard it is desirable that lattice collaborations provide in the future separately the matrix element for each $(\bar{s} d)(\bar{q} q)$ operator for $q=u, d, s$.
} 
$A_{0}$, there are then in total 16 independent matrix elements, seven of which are the ones of the SM four-quark operators and one the chromo-magnetic dipole matrix element. In the amplitude $A_{2}$, further simplifications arise as the chromomagnetic dipole operator cannot generate a $\Delta I=3 / 2$ transition, neither can an operator of the form $O_{X A B}^{u}+O_{X A B}^{d}$, leaving only five linearly independent matrix elements, three of which are present in the SM. We write the number of total matrix elements in the $I=0,2$ amplitudes as $16_{0}+5_{2}$. In "Appendix B", we specify a non-redundant basis for them.

By now these matrix elements are known with varying accuracy:

- First lattice calculations for the $7_{0}+3_{2}$ matrix elements ${ }^{5}$ generated in the SM have recently been performed by the RBC-UKQCD collaboration $[15,16]$. These results are in good agreement with the pattern of matrix elements of the relevant QCD and QED penguin operators obtained in the DQCD approach [19,50-52].

- The $K \rightarrow \pi \pi$ matrix element of the chromo-magnetic dipole operator is presently not accessible directly on the lattice, but can only be estimated by relating it to the analogous $K \rightarrow \pi$ matrix element via $S U$ (3) chiral symmetry [41]. Recently, the $K \rightarrow \pi \pi$ matrix element of this operator has been calculated directly for the first time in the DQCD approach in the $S U(3)$ chiral limit [42]. Both results are in good agreement with each other and show that the relevant matrix element is by a factor of three to four smaller than previously expected in the chiral quark model [53], thereby decreasing the impact of these operators on $\varepsilon^{\prime} / \varepsilon$. Nevertheless there are NP scenarios where they play an important role (see e.g. [36,54]).

- The matrix elements of the remaining $8_{0}+2_{2}$ linearly independent BSM four-quark matrix elements in Table 5 have only been calculated very recently in DQCD in the $S U$ (3) chiral limit [43] and it will still take some time before corresponding results in lattice QCD will be available. Yet already these approximate results from DQCD can teach us a lot about the relevance of various operators. The scalar and tensor operators $X=S, T$ belong to this group and their matrix elements cannot be expressed in terms of the SM ones.

We give the numerical values of all matrix elements in "Appendix B".

To summarize, there are three classes of matrix elements that can play a role in $\varepsilon^{\prime} / \varepsilon$,

- the matrix elements present in the SM,

\footnotetext{
5 Note that the 10 operators in the traditional SM basis are not linearly independent and correspond only to 7 linearly independent operators for $N_{f}=3$ [8].
}

- the chromo-magnetic dipole matrix element,

- the matrix elements of BSM scalar and tensor operators.

These three classes are indicated at the bottom of the sketch in Fig. 1.

\subsection{Renormalization group evolution below the} electroweak scale

In the previous subsection, we have seen that $15+15^{\prime}$ fourquark operators in $\mathcal{H}_{\Delta S=1}^{(3)}$ can contribute to $K \rightarrow \pi \pi$ at the scale $\mu$. However, additional four-quark operators are present in the five-flavour Hamiltonian $\mathcal{H}_{\Delta S=1}^{(5)}$ at $\mu_{\text {ew }}$, namely the four-quark operators with flavour structures $(\bar{s} d)(\bar{q} q)$ where $q=c$ and $b$. They can contribute to $\varepsilon^{\prime} / \varepsilon$ indirectly if they undergo QCD and/or QED RG mixing with $q=u, d$ operators. The same is true for the operators with $q=s$ that were already present for $N_{f}=3$, but did not contribute directly (at least in our approximation). In principle, also semi-leptonic operators can contribute, since they mix under QED into four-quark operators, but we will neglect them in the following, since they are typically strongly constrained from semi-leptonic kaon decays (as demonstrated for leptoquark models in [40]).

To evolve the Wilson coefficients from $\mu_{\text {ew }}$ down to the scale $\mu$ where the matrix elements are evaluated, the anomalous dimension matrices (ADMs) are required. The QCD and QED one-loop ADMs for the linearly independent set of four-quark and dipole operators can be extracted from the literature [55-57] and we have implemented them in the open source tool wilson [58] that allows to solve the RG equations numerically.

Inspection of the RG mixing reveals that

- The vector operators $O_{V A B}^{c, b}$ and their colour-flipped counterparts, as well as the operators $O_{S A B}^{s, d}$ with $A \neq B$, mix into $O_{V A B}^{u, d}$ at one loop in QCD and QED, specifically into the QCD and QED penguin operators present in the SM.

- For scalar and tensor operators $O_{X A A}^{q}(X=S$ or $T)$ there is instead no mixing among operators with different $q$. This implies in particular that the operators $O_{X A A}^{s, c, b}$ cannot mix into four-quark operators that have non-vanishing $K \rightarrow \pi \pi$ matrix elements. However, they do mix at one loop in QCD into the chromo-magnetic dipole operators $O_{8 g}^{(\prime)}$ and in QED into the electro-magnetic ones.

Taking these observations into account, we can identify for a given chirality five qualitatively different classes of NP models where different $K \rightarrow \pi \pi$ matrix elements are relevant: 
Class A Models with NP represented by the operators

$O_{V L L}^{q}, \quad \widetilde{O}_{V L L}^{q}, \quad O_{V L R}^{q}, \quad \widetilde{O}_{V L R}^{q}, \quad(q=u, c, b)$

and

$O_{V L L}^{q}, \quad O_{V L R}^{q}, \quad O_{S L R}^{q}, \quad(q=d, s)$

as well as their chirality-flipped counterparts at the electroweak scale contribute to $\varepsilon^{\prime} / \varepsilon$ via operators whose matrix elements can be written as linear combinations of the matrix elements of SM operators that were calculated in lattice QCD. Note that operators with $q=s, c, b$ contribute via RG mixing into operators with $q=u, d$ and only the matrix elements of the latter are related to matrix elements of the SM operators. Therefore NP contributions in this class of models presently rely on lattice QCD calculations $[15,16]$, which are supported by DQCD results.

Class B Models with NP represented by the operators

$O_{S L L}^{c, b}, \quad \widetilde{O}_{S L L}^{c, b}, \quad O_{T L L}^{c, b}, \quad \widetilde{O}_{T L L}^{c, b}$

and

$O_{8 g}, \quad O_{S L L}^{S}, \quad O_{T L L}^{S}$,

as well as their chirality-flipped counterparts only contribute to $\varepsilon^{\prime} / \varepsilon$ through RG mixing into the chromo-magnetic dipole operators. ${ }^{6}$ The relevant matrix element has been calculated recently by lattice QCD [41] and DQCD [42].

Class C Models with NP represented by the operators with the flavour structure $(\bar{s} d)(\bar{u} u)$

$O_{S L L}^{u}, \quad \widetilde{O}_{S L L}^{u}, \quad O_{T L L}^{u}, \quad \widetilde{O}_{T L L}^{u}$

as well as their chirality-flipped counterparts contribute via BSM matrix elements [43] or the chromo-magnetic dipole matrix elements $[41,42]$. None of these matrix elements can be expressed in terms of the ones of SM four-quark operators.

Class D Models with NP represented by the operators with the flavour structure $(\bar{s} d)(\bar{d} d)$

$O_{S L L}^{d}, \quad O_{T L L}^{d}$

as well as their chirality-flipped counterparts contribute via BSM matrix elements [43] or the chromo-magnetic dipole matrix element $[41,42]$.

Class E Models with NP represented by the operators with the flavour structure $(\bar{s} d)(\bar{u} u)$

$O_{S L R}^{u}, \quad \widetilde{O}_{S L R}^{u}$,

as well as their chirality-flipped counterparts contribute exclusively via BSM matrix elements [43] to the $I=0$ amplitude. The $I=2$ matrix elements can instead be expressed in terms of the SM ones.

$\overline{6}$ As stated above, we neglect electro-magnetic dipole operators.
There are $37+37^{\prime}$ operators in Classes A-E. The only remaining $4+4^{\prime}$ operators in $\mathcal{H}_{\Delta S=1}^{(5)}$, namely

$O_{S L R}^{c, b}, \quad \widetilde{O}_{S L R}^{c, b}$,

and their chirality-flipped counterparts, have been omitted since they neither contribute directly nor via RG mixing to $\varepsilon^{\prime} / \varepsilon$ at the level considered.

\subsection{Master formula for $\varepsilon^{\prime} / \varepsilon$ beyond the SM}

Having both the RG evolution and all matrix elements at the low-energy scale $\mu$ for the first time at hand allowed us recently [46] to present in a letter a master formula for $\left(\varepsilon^{\prime} / \varepsilon\right)_{\text {BSM }}$ that exhibits its dependence on each Wilson coefficient at the scale $\mu_{\text {ew }}$ and consequently is valid in any theory beyond the SM that is free from non-standard light degrees of freedom below the electroweak scale. We will now discuss various ingredients and technical details which led to this formula.

The numerical analysis in [46] has been performed with the public codes flavio [59,60] and wilson [58]. In the evaluation of $\varepsilon^{\prime} / \varepsilon$ we set $\operatorname{Re} A_{0,2}$ in (2) to the measured values [61]

$\operatorname{Re} A_{0}=27.04(1) \times 10^{-8} \mathrm{GeV}$,

$\operatorname{Re} A_{2}=1.210(2) \times 10^{-8} \mathrm{GeV}$,

accounting thus for potential new physics. We use here the same convention for the normalization $(h=1)$ of the amplitudes as has been chosen for the calculation of the matrix elements of BSM operators in $[42,43]$, which differs from the one $(h=\sqrt{3 / 2})$ used by RBC-UKQCD $[15,16]$. We fix $\mu_{\mathrm{ew}}=160 \mathrm{GeV}$, close to the top-quark mass, and $\mu=1.3 \mathrm{GeV}$. Writing $\varepsilon^{\prime} / \varepsilon$ as a sum of the SM and BSM contributions,

$\frac{\varepsilon^{\prime}}{\varepsilon}=\left(\frac{\varepsilon^{\prime}}{\varepsilon}\right)_{\mathrm{SM}}+\left(\frac{\varepsilon^{\prime}}{\varepsilon}\right)_{\mathrm{BSM}}$,

the master formula of [46] for the BSM part then reads ${ }^{7}$

$\left(\frac{\varepsilon^{\prime}}{\varepsilon}\right)_{\mathrm{BSM}}=\sum_{i} P_{i}\left(\mu_{\mathrm{ew}}\right) \operatorname{Im}\left[C_{i}\left(\mu_{\mathrm{ew}}\right)-C_{i}^{\prime}\left(\mu_{\mathrm{ew}}\right)\right] \times(1 \mathrm{TeV})^{2}$,

where

$P_{i}\left(\mu_{\mathrm{ew}}\right)=\sum_{j} \sum_{I=0,2} p_{i j}^{(I)}\left(\mu_{\mathrm{ew}}, \mu\right)\left[\frac{\left\langle O_{j}(\mu)\right\rangle_{I}}{\mathrm{GeV}^{3}}\right]$,

7 Note that here we have the convention of dimensionful Wilson coefficients in (4) and (61) in contrast to [46]. Both are related by simple rescaling $C_{i}^{\text {here }}=C_{i} /(1 \mathrm{TeV})^{2}$, which is taken care of in (18), such that $P_{i}$ and $p_{i j}^{(I)}$ are the same. 
with the sum over $i$ extending over the Wilson coefficients $C_{i}$ of all operators in Classes A-E and their chirality-flipped counterparts, that is $36+36^{\prime}$ linearly independent four-quark operators and $1+1^{\prime}$ chromo-magnetic dipole operators. The $C_{i}^{\prime}$ are the Wilson coefficients of the corresponding chiralityflipped operators obtained by interchanging $P_{L} \leftrightarrow P_{R}$. The relative minus sign accounts for the fact that their $K \rightarrow \pi \pi$ matrix elements differ by a sign. Among the contributing operators are also operators present already in the SM but their Wilson coefficients in (18) include only BSM contributions.

The dimensionless coefficients $p_{i j}^{(I)}\left(\mu_{\mathrm{ew}}, \mu\right)$ include the QCD and QED RG evolution from $\mu_{\text {ew }}$ to $\mu$ for each Wilson coefficient as well as the relative suppression of the contributions to the $I=0$ amplitude due to $\operatorname{Re} A_{2} / \operatorname{Re} A_{0} \ll 1$ for the matrix elements $\left\langle O_{j}(\mu)\right\rangle_{I}$ of all the operators $O_{j}$ present at the low-energy scale, see "Appendix B". The index $j$ includes also $i$ so that the effect of self-mixing is included. The $P_{i}\left(\mu_{\mathrm{ew}}\right)$ do not depend on $\mu$ to the considered order, because the $\mu$-dependence cancels between matrix elements and the RG evolution operator. Moreover, it should be emphasized that their values are model-independent and depend only on the SM dynamics below the electroweak scale, which includes short distance contributions down to $\mu$ and the long distance contributions represented by the hadronic matrix elements. The BSM dependence enters our master formula in (18) only through the Wilson coefficients $C_{i}\left(\mu_{\mathrm{ew}}\right)$ and $C_{i}^{\prime}\left(\mu_{\mathrm{ew}}\right)$. That is, even if a given $P_{i}$ is non-zero, the fate of its contribution depends on the difference of these two coefficients. In particular, in models with exact left-right symmetry this contribution vanishes as first pointed out in [62].

The numerical values of the $P_{i}\left(\mu_{\mathrm{ew}}\right)$ are collected in the tables in "Appendix C". As seen in (19), the $P_{i}$ depend on the hadronic matrix elements $\left\langle O_{j}(\mu)\right\rangle_{I}$ and the RG evolution factors $p_{i j}^{(I)}\left(\mu_{\mathrm{ew}}, \mu\right)$. The numerical values of the hadronic matrix elements rely on lattice QCD in the case of SM operators and DQCD in the case of BSM operators as summarized above. Consequently, the uncertainties of the $P_{i}$ are of the order of 5-7\% resulting from SM matrix elements and at the level of $20 \%$ coming from BSM matrix elements.

Inspecting the results in the tables in "Appendix C" the following comments are in order.

- The large $P_{i}$ values for operators with flavour content $(\bar{s} d)(\bar{u} u)$ and $(\bar{s} d)(\bar{d} d)$ in Class A can be traced back to the large values of the matrix elements $\left\langle Q_{7,8}\right\rangle_{2}$, the dominant electroweak penguin operators in the SM, and the enhancement of the $I=2$ contributions relative to $I=0$ ones by $\omega \approx 22$.

- The small $P_{i}$ values in Class B are due to the fact that they are all proportional to $\left\langle O_{8 g}\right\rangle_{0}$, which has recently been found to be much smaller than previously expected
[41,42]. Moreover, as $\left\langle O_{8 g}\right\rangle_{2}=0$, all contributions in this class are suppressed by the factor $1 / \omega$ relative to contributions from other classes.

- The large $P_{i}$ values in Classes C-D can be traced back to the large hadronic matrix elements of scalar and tensor operators calculated recently in [43]. Due to the smallness of $\left\langle O_{8 g}\right\rangle_{0}$, the contribution of the chromo-magnetic dipole operator in Classes $\mathrm{C}-\mathrm{D}$ is negligible.

- While the $I=0$ matrix elements of the operators in Class E cannot be expressed in terms of SM ones, the $I=2$ matrix elements can, and the large $P_{i}$ values can be traced back to the large SM matrix elements $\left\langle Q_{7,8}\right\rangle_{2}$.

\subsection{Matching from SMEFT onto $\Delta S=1$ EFT}

The SMEFT is a convenient description of BSM scenarios that feature a large gap between the NP and the electroweak scales, $\mu_{\Lambda} \gg \mu_{\text {ew }}$. This implies that there are only the known SM fields below $\mu_{\Lambda}$ and it is assumed that the Higgs doublet is in the linear representation. The SM dimension-four Lagrangian is supplemented by a tower of local operators

$\mathcal{L}_{\mathrm{SMEFT}}=\mathcal{L}_{\mathrm{dim}-4}+\sum_{k} \mathcal{C}_{k} \mathcal{O}_{k}$,

that are invariant under the $\mathrm{SM}$ gauge group $G_{\mathrm{SM}}=$ $S U(3)_{c} \otimes S U(2)_{L} \otimes U(1)_{Y}$ to describe physics below $\mu_{\Lambda}$ around $\mu_{\mathrm{ew}}$.

The SMEFT operators and accordingly their Wilson coefficients are defined in terms of the gauge and fermion fields in the unbroken phase of the SM, see also "Appendix D" for notation and definitions. In contrast to the $\Delta S=1$ EFT discussed above, there is no preferred weak basis for the (massless) fermion fields in SMEFT and the would-be mass basis is not $S U(2)_{L}$ invariant. Instead, in the following we use the freedom of $S U$ (3)-flavour rotations to work in a weak basis where the running down-type quark mass matrix is diagonal at the electroweak scale (cf. [63]).

At the electroweak scale $\mu_{\mathrm{ew}}$, the matching of SMEFT at the dimension-six level will only generate a subset of the $\Delta S=1$ operators introduced in "Appendix A", because the SM gauge group $G_{\mathrm{SM}}$ is more restrictive than $S U(3)_{c} \otimes$ $U(1)_{Q}$. Since flavour is not conserved by the RG mixing under the $S U(2)_{L}$-gauge and Higgs-Yukawa interactions the SMEFT operators cannot be classified in terms of flavour quantum numbers. Nevertheless, it is instructive to consider which operators in SMEFT contribute to $\Delta S=1$ transitions when matched at tree level onto $\mathcal{H}_{\Delta S=1}^{(5)}$ at the scale $\mu_{\text {ew }}$.

The matching of SMEFT onto the $\Delta S=1$ EFT with five active quark flavours yields relations between Wilson coefficients ${ }^{8}$ of both EFTs $[64,65]$. Here we focus on effects from three classes of operators:

\footnotetext{
${ }_{8}$ We denote Wilson coefficients of SMEFT by caligraphic $\mathcal{C}_{k}$ and of low-energy EFTs by $C_{k}$.
} 
Table 1 Number of linearly independent four-quark operators with flavour content $(\bar{s} d)\left(\bar{u}_{i} u_{i}\right)$ and $(\bar{s} d)\left(\bar{d}_{i} d_{i}\right)$ in the $\Delta S=1$ EFT with $N_{f}=5$ that contribute to $\varepsilon^{\prime} / \varepsilon$ (first row). Number of non-vanishing matching contributions from SMEFT due to four-quark operators and modified right-handed $W^{ \pm}$couplings for dimension-six operators at tree level (second row)

\begin{tabular}{|c|c|c|c|c|c|c|}
\hline & $u_{i}=u$ & $u_{i}=c$ & $d_{i}=d$ & $d_{i}=s$ & $d_{i}=b$ & $\Sigma$ \\
\hline$\Delta S=1 \mathrm{EFT}$ & $10+10^{\prime}$ & $8+8^{\prime}$ & $5+5^{\prime}$ & $5+5^{\prime}$ & $8+8^{\prime}$ & $36+36^{\prime}$ \\
\hline SMEFT & $9+9^{\prime}$ & $8+8^{\prime}$ & $3+3^{\prime}$ & $3+3^{\prime}$ & $4+4^{\prime}$ & $27+27^{\prime}$ \\
\hline
\end{tabular}

- four-quark operators,

- $\psi^{2} H^{2} D$ operators describing modified $Z^{0}$ or $W^{ \pm}$couplings, and

- chromo-magnetic dipole operators.

The matching conditions for these operators are collected in "Appendix E". We omit the effects from semi-leptonic operators that have been analysed in the context of leptoquark models [40] and are expected to be constrained more strongly by semi-leptonic kaon decays rather than $\varepsilon^{\prime} / \varepsilon$. Furthermore we omit effects of purely bosonic operators, which can induce a sizable contribution to $\varepsilon^{\prime} / \varepsilon$ through RG effects as discussed in [66].

A non-trivial consequence of SMEFT is that none of the operators $O_{S L R}^{u_{i}}, O_{S L L}^{d_{i}}, O_{T L L}^{d_{i}}$, or their chirality- and colourflipped counterparts, are generated in the low-energy EFT in the tree-level matching of SMEFT four-quark operators. The reason is that these operators conserve only electric charge, but not hypercharge. Only the operator $\widetilde{O}_{S L R}^{u}$ eventually contributes to $\varepsilon^{\prime} / \varepsilon$, namely through the right-handed $W^{ \pm}$ coupling discussed in "Appendix E.2". This contribution is not subject to the hypercharge constraint, as it only arises after electroweak symmetry breaking. Below $\mu_{\text {ew }}$ this leads to vanishing Wilson coefficients of $9+9^{\prime}$ linearly independent operators in the $\Delta S=1$ EFT with $N_{f}=5$, reducing the number of non-redundant $\Delta S=1$ four-quark operators that contribute to $\varepsilon^{\prime} / \varepsilon$ from $36+36^{\prime}$ to $27+27^{\prime}{ }^{9}$ At the one-loop level, QCD and QED running from $\mu_{\mathrm{ew}}$ down to $\mu$ does not re-generate these operators. This is summarized in Table 1. Consequently, in SMEFT the number of linearly independent operators that contribute directly to $\varepsilon^{\prime} / \varepsilon$ via non-vanishing $K \rightarrow \pi \pi$ matrix elements is reduced from $15+15^{\prime}$ to $12+12^{\prime}$, out of which only $5+5^{\prime}$ are non-standard. The chromo-magnetic dipole operators are not subject to these considerations and their number equals in SMEFT and $\Delta S=1$ EFT.

Consequently, in SMEFT only the operators in Classes AC in (8)-(12) contribute to $\varepsilon^{\prime} / \varepsilon$ through four-quark operators, and a single operator from Class $\mathrm{E}$ in (14) (and its chiralityflipped counterpart) through the right-handed $W^{ \pm}$coupling.

\footnotetext{
9 Note that (76)-(92) contain operators that are related by Fierz transformations. These allow to remove 12 of the 68 operators for $N_{f}=5$ appearing on the left-hand side of these equations.
}

Inspecting the matching relations listed in "Appendix E", these three classes, expressed in terms of the SMEFT operators of Tables 11 and 12, are as follows

\section{Class A}

$$
\begin{aligned}
& \mathcal{O}_{q q}^{(1,3)}, \quad \mathcal{O}_{q u}^{(1,8)}, \quad \mathcal{O}_{q d}^{(1,8)}, \quad \mathcal{O}_{u d}^{(1,8)}, \quad \mathcal{O}_{d d}, \\
& \mathcal{O}_{H q}^{(1,3)}, \quad \mathcal{O}_{H d} .
\end{aligned}
$$

\section{Class B and C}

$\mathcal{O}_{d G}, \quad \mathcal{O}_{\text {quqd }}^{(1,8)}$.

Class E

$\mathcal{O}_{\text {Hud }}$.

It should be noted that while the matching conditions in Sect. 2.4 are at the electroweak scale, the SMEFT operators are generated by some BSM dynamics at a much higher scale $\mu_{\Lambda}$ and in explicit models RG evolution in the SMEFT from $\mu_{\Lambda}$ to $\mu_{\text {ew }}$ has to be considered [67-69]. The RG evolution does not only change the values of the Wilson coefficients through self-mixing of a given operator but also through mixing of other operators, in particular those that do not contribute directly to $\varepsilon^{\prime} / \varepsilon$ at tree-level. The mixing is further complicated due to the flavour structure of the ADMs and can give rise to complex correlation patterns between observables of various quark and lepton flavour sectors. In "Appendix F", we list the classes of operators that mix into operators contributing to the tree-level matching onto the $\Delta S=1 \mathrm{EFT}$ and can thus be relevant for $\varepsilon^{\prime} / \varepsilon$.

\section{Model-independent constraints in SMEFT}

In specific NP models, $\mathrm{CP}$-violating effects that can manifest themselves in $\varepsilon^{\prime} / \varepsilon$ are constrained by other CP-odd observables, such as in $\Delta S=2\left(K^{0}-\bar{K}^{0}\right.$ mixing $), \Delta C=2\left(D^{0}\right.$ $\bar{D}^{0}$ mixing), in electric dipole moments (EDM), or in semileptonic kaon decays. In the low-energy EFT, such effects cannot be discussed on a model-independent basis, since the operators with different flavour quantum numbers are completely independent. In SMEFT however, such correlations can arise in two different ways, 
Table 2 Effective scales of SMEFT operators contributing to $\varepsilon_{K}$ and CP violation in $D^{0}-\bar{D}^{0}$ mixing, defined as in (25) and (31), respectively. These scales give an indication of the sensitivity to the individual oper- ators. Note however that the normalization is different for $\Delta S=2$ and $\Delta C=2$

\begin{tabular}{|c|c|c|c|c|c|c|c|c|}
\hline $\mathcal{C}_{i}$ & $\sigma_{i}$ & $\Lambda_{i}$ & $\mathcal{C}_{i}$ & $\sigma_{i}$ & $\Lambda_{i}$ & $\mathcal{C}_{i}$ & $\sigma_{i}$ & $\Lambda_{i}$ \\
\hline \multicolumn{9}{|l|}{$\Delta S=2$} \\
\hline$\left[\mathcal{C}_{q q}^{(1)}\right]_{2121}$ & - & 13.3 PeV & {$\left[\mathcal{C}_{q d}^{(1)}\right]_{2121}$} & + & 104.6 PeV & \multirow[t]{3}{*}[\mathcal{C}_{dd}]{$_{2121}$} & \multirow[t]{3}{*}{-} & \multirow[t]{3}{*}{ 13.3 PeV } \\
\hline$\left[\mathcal{C}_{q q}^{(3)}\right]_{2121}$ & - & $13.3 \mathrm{PeV}$ & {$\left[\mathcal{C}_{q d}^{(8)}\right]_{2121}$} & + & 126.5 PeV & & & \\
\hline$\Delta C=2$ & & & & & & & & \\
\hline$\left[\widehat{\mathcal{C}}_{q q}^{(1)}\right]_{1212}$ & - & $14.1 \mathrm{PeV}$ & {$\left[\widehat{\mathcal{C}}_{q u}^{(1)}\right]_{1212}$} & + & $29.2 \mathrm{PeV}$ & \multirow[t]{2}{*}[\widehat{\mathcal{C}}_{uu}]{$_{1212}$} & \multirow[t]{2}{*}{-} & \multirow[t]{2}{*}{ 14.1 PeV } \\
\hline$\left[\widehat{\mathcal{C}}_{q q}^{(3)}\right]_{1212}$ & - & 14.1 PeV & {$\left[\widehat{\mathcal{C}}_{q u}^{(8)}\right]_{1212}$} & + & $33.3 \mathrm{PeV}$ & & & \\
\hline
\end{tabular}

- by $S U(2)_{L}$ relations between operators involving lefthanded quark doublets that require a CKM rotation to go to the mass basis for the up- or down-type quarks,

- by flavour-dependent RG effects due to the mixing of operators in SMEFT given in Sect. 2.4.

In this section, we concentrate on effects of the first type, leading to model-independent constraints on Wilson coefficients of operators contributing to $\varepsilon^{\prime} / \varepsilon$.

\section{$3.1 \Delta S=2$}

The parameter $\varepsilon_{K}$ measures indirect $\mathrm{CP}$ violation in the $\Delta S=2$ process of $K^{0}-\bar{K}^{0}$ mixing. At the electroweak scale only four linear combinations of the five ${ }^{10}$ SMEFT operators

$\left[\mathcal{O}_{q q}^{(1)}\right]_{2121}, \quad\left[\mathcal{O}_{q q}^{(3)}\right]_{2121}, \quad\left[\mathcal{O}_{q d}^{(1)}\right]_{2121},\left[\mathcal{O}_{q d}^{(8)}\right]_{2121}, \quad\left[\mathcal{O}_{d d}\right]_{2121}$,

match onto the $\Delta S=2$ EFT at tree level in the weak basis in which the down-type quark mass matrix is diagonal. The other four operators present in the $\Delta S=2$ EFT violate hypercharge and are thus not generated at tree level [64].

The quantitative effect of these operators can be understood by writing $\varepsilon_{K}$ as a function of their Wilson coefficients with approximate numerical coefficients,

$$
\frac{\varepsilon_{K}}{\varepsilon_{K}^{\mathrm{SM}}} \approx 1+\sum_{i} \sigma_{i} \Lambda_{i}^{2} \operatorname{Im} \mathcal{C}_{i}\left(\mu_{\mathrm{ew}}\right)
$$

where $\sigma_{i}= \pm 1$. Similarly to the $P_{i}$ in the master formula (18) for $\varepsilon^{\prime} / \varepsilon$, the effective scales $\Lambda_{i}$ give an indication of the sensitivity of $\varepsilon_{K}$ to each Wilson coefficient; we list their

$\overline{10}$ We do not count redundant operators such as $\left[\mathcal{O}_{q d}^{(1,8)}\right]_{1212} \equiv$ $\left[\mathcal{O}_{q d}^{(1,8)}\right]_{2121}^{\dagger}$, but adopt the basis of non-redundant operators defined in [70]. Contributions from $\psi^{2} H^{2} D$ operators corresponding to modified $Z^{0}$ and $W^{ \pm}$couplings arise at one-loop from top-quark Yukawa mixing [24] and those from $h^{0}$ couplings count as beyond dimension $\operatorname{six}[65]$. numerical values in Table 2. They have been obtained with flavio [59] and wilson [58] using the $\Delta S=2$ hadronic matrix elements from lattice QCD by RBC-UKQCD [71,72] (cf. results from the ETM [73] and SWME [74] collaborations), which are supported by DQCD results [75].

As the SM describes the experimental value of $\varepsilon_{K}$ rather well, $\operatorname{Im} \mathcal{C}_{i}\left(\mu_{\text {ew }}\right)$ corresponding to the largest $\Lambda_{i}$ must be suppressed most strongly, thereby probing the largest NP scales. Given the experimental measurement and theory uncertainty of this ratio (25) can be used to constrain SMEFT Wilson coefficients from $\varepsilon_{K}$ in phenomenological analyses.

Given these huge scales probed by $\varepsilon_{K}$, any model predicting sizable direct $\mathrm{CP}$ violation in $\Delta S=1$ can only be viable if it does not induce too large contributions to indirect $\mathrm{CP}$ violation in $\Delta S=2$.

As discussed above, an important source of constraints are $S U(2)_{L}$ relations between operators with left-handed quark fields, involving a CKM rotation between the mass bases for up- and down-type quarks. The first four operators in Table 2 are a prime example of this effect. $\left[\mathcal{C}_{q q}^{(1,3)}\right]_{2121}$ contribute to the matching of $C_{V L L}^{u_{i}}$ and $\left[\mathcal{C}_{q d}^{(1,8)}\right]_{2121}$ to the matching of $C_{V R L}^{u_{i}}$, as seen from the matching conditions in Sect. 2.4. For both cases $i=1,2$, the suppression by the Cabibbo angle $V_{u s} V_{u d}^{*} \sim V_{c s} V_{c d}^{*} \sim 0.23$ is of first order in $\varepsilon^{\prime} / \varepsilon$ and furthermore the operators with $i=2$ have no direct $K \rightarrow \pi \pi$ matrix elements, which introduces for them another suppression of $\alpha_{s, e} /(4 \pi)$ from RG mixing in $\varepsilon^{\prime} / \varepsilon$ compared to $i=1$. When considering NP effects in only a single operator, clearly the strong constraint from $\varepsilon_{K}$ excludes any visible effect in $\varepsilon^{\prime} / \varepsilon$ induced by imaginary parts of these Wilson coefficients.

We finally note that, as emphasized in [29], new phases could have an impact not only on $\varepsilon_{K}$, but also on the mass difference in $K^{0}-\bar{K}^{0}$ mixing, $\Delta M_{K}$. The point is that $\Delta M_{K}$ is proportional to the real part of the square of a complex coefficient $C_{i}$, so a new phase modifying its imaginary part will quite generally decrease the value of $\Delta M_{K}$ relative to the SM estimate simply because 
$\left(\Delta M_{K}\right)_{i}^{\mathrm{BSM}}=c\left[\left(\operatorname{Re} C_{i}\right)^{2}-\left(\operatorname{Im} C_{i}\right)^{2}\right]$,

with $c$ being positive. The uncertainty in the SM estimate of $\Delta M_{K}$ is unfortunately still very large [76] so that we cannot presently decide whether a positive or negative NP contribution to $\Delta M_{K}$ - if any - is required and the constraints on the NP scale are weaker than for $\varepsilon_{K}$. Future lattice QCD calculations of long distance contributions to $\Delta M_{K}$ could help in this respect $[77,78]$. In DQCD they are found to amount to $20 \pm 10 \%$ of the measured $\Delta M_{K}[52,79]$. In the case of $\varepsilon_{K}$ such long distance contributions to $\varepsilon_{K}$ are below $10 \%$ and have been reliably calculated in $[16,80,81]$.

\section{$3.2 \Delta C=2$}

Although the SM contribution to the $D^{0}-\bar{D}^{0}$ mixing amplitude is dominated by poorly known long-distance contributions, the structure of the CKM matrix implies that the SM contribution to $\mathrm{CP}$ violation in mixing can at most reach the percent level [82]. This fact can be used to constrain the imaginary part of the mixing amplitude.

For processes with external up-type quarks, it is more convenient to use a weak basis for SMEFT Wilson coefficients where the up-type rather than the down-type quark mass matrix is diagonal. ${ }^{11}$ We will denote the Wilson coefficients in this basis with a hat. The hatted Wilson coefficients are related to the unhatted ones by CKM rotations of indices corresponding to left-handed quark doublets,

$$
\begin{aligned}
{\left[\widehat{\mathcal{C}}_{q q}^{(1,3)}\right]_{i j k l} } & =V_{i a} V_{j b}^{*} V_{k c} V_{l d}^{*}\left[\mathcal{C}_{q q}^{(1,3)}\right]_{a b c d}, \\
{\left[\widehat{\mathcal{C}}_{q u}^{(1,8)}\right]_{i j k l} } & =V_{i a} V_{j b}^{*}\left[\mathcal{C}_{q u}^{(1,8)}\right]_{a b k l}, \\
{\left[\widehat{\mathcal{C}}_{u u}\right]_{i j k l} } & =\left[\mathcal{C}_{u u}\right]_{i j k l} .
\end{aligned}
$$

Then, analogously to $\varepsilon_{K}$, at the electroweak scale four linear combinations of five SMEFT operators contribute to $\Delta C=2$ transitions, namely

$$
\begin{gathered}
{\left[\widehat{\mathcal{O}}_{q q}^{(1)}\right]_{1212},\left[\widehat{\mathcal{O}}_{q q}^{(3)}\right]_{1212}, \quad\left[\widehat{\mathcal{O}}_{q u}^{(1)}\right]_{1212},} \\
{\left[\widehat{\mathcal{O}}_{q u}^{(8)}\right]_{1212}, \quad\left[\widehat{\mathcal{O}}_{u u}\right]_{1212} .}
\end{gathered}
$$

A correlation of $\varepsilon^{\prime} / \varepsilon$ and $D^{0}-\bar{D}^{0}$ mixing arises only for the operators $\mathcal{O}_{q q}^{(1,3)}$ and $\mathcal{O}_{q u}^{(1,8)}$ as can be seen from (76)-(92).

From a global fit to $D^{0}$ decays, the HFLAV collaboration directly determines the physical parameters of the $D^{0}-\bar{D}^{0}$ mixing amplitude,

$$
x_{12}=\frac{2\left|M_{12}\right|}{\Gamma}, \quad y_{12}=\frac{\left|\Gamma_{12}\right|}{\Gamma}, \quad \phi_{12}=\arg \frac{M_{12}}{\Gamma_{12}} .
$$

\footnotetext{
11 This basis is denoted Warsaw up in the WCxf standard [63]
}

Their fit result can be expressed as an approximately Gaussian constraint on the purely CP-violating parameter [83]

$x_{12}^{\mathrm{Im}} \equiv x_{12} \sin \phi_{12}=(0 \pm 2.4) \times 10^{-4}$.

Similarly to the discussion of $\varepsilon_{K}$ above, we can write $x_{12}^{\mathrm{Im}}$ as a linear function of SMEFT Wilson coefficients at $\mu_{\mathrm{ew}}$,

$\frac{x_{12}^{\operatorname{Im}}}{10^{-4}} \approx \sum_{i} \sigma_{i} \Lambda_{i}^{2} \operatorname{Im} \widehat{\mathcal{C}_{i}}$.

The effective sensitivity scales $\Lambda_{i}$ are given in Table 2 . They have been evaluated with flavio [59] and wilson [58] using the $\Delta C=2$ hadronic matrix elements from lattice QCD by the ETM collaboration [73].

Similarly to the $\Delta S=2$ case, we see that the four Wilson coefficients $\left[\widehat{\mathcal{C}}_{q q}^{(1,3)}\right]_{1212}$ and $\left[\widehat{\mathcal{C}}_{q u}^{(1,8)}\right]_{1212}$ individually cannot give a visible effect in $\varepsilon^{\prime} / \varepsilon$ without generating excessive contributions to $\mathrm{CP}$ violation in $\Delta C=2$.

\subsection{Interplay of $\Delta S=2$ and $\Delta C=2$}

While individually, $\Delta S=2$ and $\Delta C=2$ only constrain seven linear combinations of SMEFT Wilson coefficients contributing to the $\Delta S=1$ matching, combining them leads to a much more powerful constraint. This is because the Wilson coefficients $\left[\mathcal{C}_{q q}^{(1,3)}\right]_{1212}$ and $\left[\widehat{\mathcal{C}}_{q q}^{(1,3)}\right]_{1212}$ are related by CKM rotations:

$\left[\widehat{\mathcal{C}}_{q q}^{(1,3)}\right]_{1212}=V_{u i} V_{c j}^{*} V_{u k} V_{c l}^{*}\left[\mathcal{C}_{q q}^{(1,3)}\right]_{i j k l}$,

$\left[\mathcal{C}_{q q}^{(1,3)}\right]_{1212}=V_{i d}^{*} V_{j s} V_{k d}^{*} V_{l s}\left[\widehat{\mathcal{C}}_{q q}^{(1,3)}\right]_{i j k l}$.

Consequently, for any given operator of this type, it is impossible to avoid both the contribution to $\Delta S=2$ and $\Delta C=2$ at the same time (cf. the general discussion in [84]). Indeed, switching on individual operators in either of the two bases at $\mu_{\mathrm{ew}}$, it turns out they all lead to an excessive contribution to either $\varepsilon_{K}$ or $x_{12}^{\mathrm{Im}}$ when generating a visible effect in $\varepsilon^{\prime} / \varepsilon$. This is illustrated in Fig. 2, showing the suppression scales $\Lambda_{i}$ for $\varepsilon_{K}$ and $x_{12}^{\operatorname{Im}}$ (as defined in (25), (31)) and comparing it to the analogous scale for $\varepsilon^{\prime} / \varepsilon$, defined as

$$
\frac{\left(\varepsilon^{\prime} / \varepsilon\right)_{\mathrm{BSM}}}{10^{-3}} \approx \sum_{i} \sigma_{i} \Lambda_{i}^{2} \operatorname{Im}\left[\mathcal{C}_{q q}^{(1,3)}\right]_{i}
$$

in the two different bases where either the down-type or the up-type quark mass matrix is diagonal. While in the former basis $\varepsilon_{K}$ and in the latter basis $x_{12}^{\mathrm{Im}}$ is only sensitive to a single coefficient, the other observable probes all the other coefficients, always being much more sensitive than $\varepsilon^{\prime} / \varepsilon$.

We finally note that, in principle, since each of the observables only probes a single direction in the space of Wilson coefficients, cancellations could be arranged that remove these constraints. In view of the severeness of the constraints 


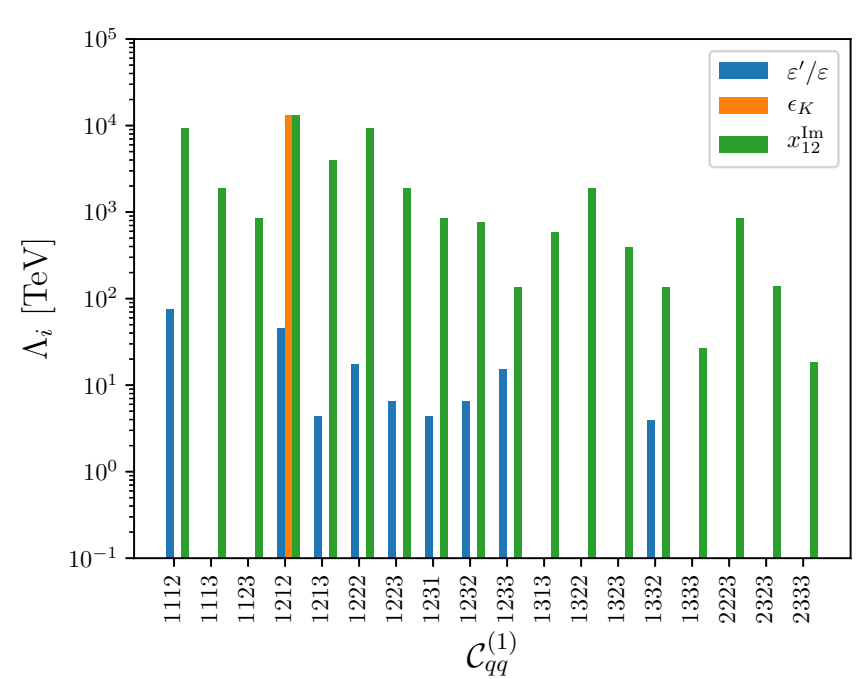

Fig. 2 Effective scales $\Lambda_{i}$ of the Wilson coefficients $\mathcal{C}_{q q}^{(1)}$ for $\varepsilon_{K}$ (orange), $D^{0}-\bar{D}^{0}$ mixing (green) and the NP contribution to $\varepsilon^{\prime} / \varepsilon$ (blue), parametrized as in (25), (31), and (34), respectively. The left panel shows the values in the "unhatted" basis where the down-type quark mass matrix is diagonal, the right panel in the "hatted" basis where the up-

and the fact that delicate cancellations are not invariant under the RG evolution, we consider such cancellations unrealistic.

\subsection{Neutron electric dipole moment}

Since $\varepsilon^{\prime} / \varepsilon$ probes CP violation associated to the first two generations of quarks, it is natural to ask whether there is any constraint from the electric dipole moment (EDM) of the neutron, which is a sensitive probe of flavour-diagonal $\mathrm{CP}$ violation involving up and down quarks. In principle, CP-violating four-quark operators can directly induce a neutron EDM. Correlations of the neutron EDM with $\varepsilon^{\prime} / \varepsilon$ from these operators have been considered recently in $[37,85]$; they require the knowledge of the matrix elements of these operators, which are relatively poorly known.

Here we focus instead on CP violation induced by dipole operators, i.e. the EDMs and chromo-EDMs (CEDMs) of the up and down quarks. Their contribution to the neutron EDM can be written as ${ }^{12}$

$d_{n}=g_{T}^{u} d_{u}+g_{T}^{d} d_{d}+\tilde{\rho}_{u} \tilde{d}_{u}+\tilde{\rho}_{d} \tilde{d}_{d}$

The tensor charges $g_{T}^{u, d}$ are nowadays accessible in lattice QCD with an accuracy of (5-10)\% [86-89], while the matrix elements $\tilde{\rho}_{u, d}$ of the CEDMs are only known roughly from methods like light-cone sum rules $[90,91]$. The quark

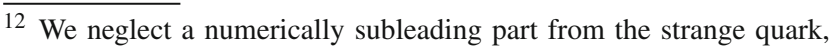
since $g_{T}^{s} \ll g_{T}^{u, d}$, and assume that the contribution from the strange quark CEDM can be neglected as well.
}

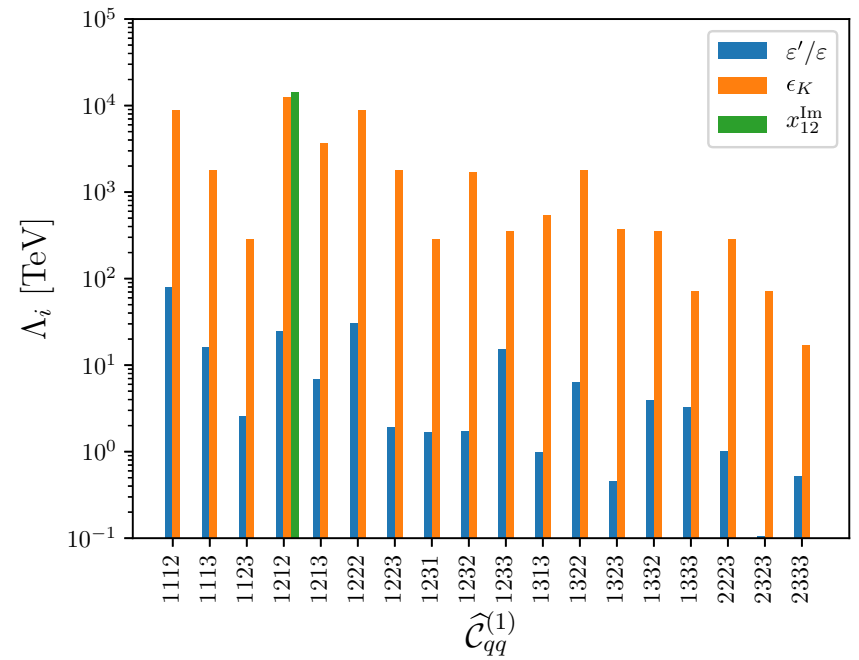

type quark mass matrix is diagonal. Only non-redundant flavour-index combinations are shown. Coefficients that do not generate a visible effect in either observable have been omitted. The scales corresponding to $\mathcal{C}_{q q}^{(3)}$ are not shown but are very similar

(C)EDMs are simply the imaginary parts of the Wilson coefficients of the flavour-diagonal dipole operators at the hadronic scale, ${ }^{13}$

$d_{q}=2 m_{q} \operatorname{Im} C_{7 \gamma}^{q q}, \quad g_{s} \tilde{d}_{q}=2 m_{q} \operatorname{Im} C_{8 g}^{q q}$,

with the effective Hamiltonian

$$
\begin{aligned}
\mathcal{H}_{\Delta F=0}= & -\sum_{q=u, d}\left[C_{7 \gamma}^{q q} m_{q}\left(\bar{q} \sigma^{\mu \nu} P_{R} q\right) F_{\mu \nu}\right. \\
& \left.+C_{8 g}^{q q} m_{q}\left(\bar{q} \sigma^{\mu v} P_{R} T^{A} q\right) G_{\mu \nu}^{A}+\text { h.c. }\right] .
\end{aligned}
$$

Below the electroweak scale, the dipole operators receive RG-induced contributions via QCD and QED penguin diagrams from operators with chirality structure LRLR,

$\begin{aligned} O_{X A A}^{q q p p} & =\left(\bar{q}^{i} \Gamma_{X} P_{A} q^{i}\right)\left(\bar{p}^{j} \Gamma_{X} P_{A} p^{j}\right), \\ \widetilde{O}_{X A A}^{q q p p} & =\left(\bar{q}^{i} \Gamma_{X} P_{A} q^{j}\right)\left(\bar{p}^{j} \Gamma_{X} P_{A} p^{i}\right),\end{aligned}$

where $X=S, T$ and $A=L, R$. In tree-level matching from SMEFT at $\mu_{\text {ew }}$, such operators are only generated from the SMEFT operators $\mathcal{O}_{\text {quqd }}^{(1,8)}$, similarly to the operators $C_{S A A}^{u_{i}}$ and $C_{T A A}^{u_{i}}$ in the $\Delta S=1$ matching in Sect. 2.4. Via CKM rotations, many of the operators in (38) are thus related to $\Delta S=1$ operators.

13 Note that the signs on the right-hand sides of (36) depend on the sign convention for the covariant derivative. We use $D_{\mu}=\partial_{\mu}+i e Q_{f} A_{\mu}+$ $i g_{s} G_{\mu}^{a} T^{a}$. Our convention for $\sigma^{\mu \nu}$ is $\sigma^{\mu \nu}=\frac{i}{2}\left[\gamma_{\mu}, \gamma_{\nu}\right]$. 


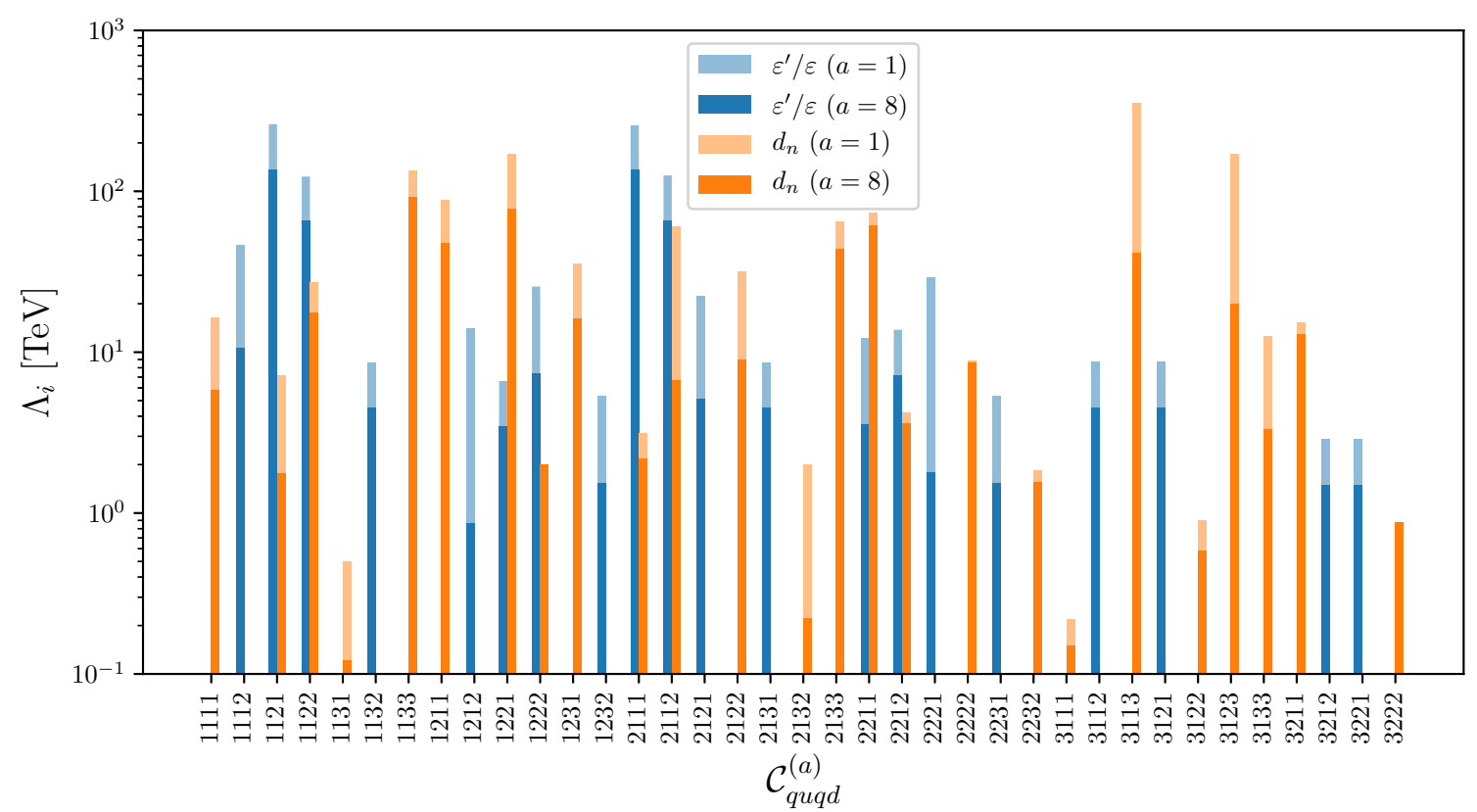

Fig. 3 Effective scales $\Lambda_{i}$ for the neutron EDM [orange] and the NP contribution to $\varepsilon^{\prime} / \varepsilon$ [blue], parametrized as in (39) and (41), respectively. Only non-redundant index combinations are shown. Coefficients

Analogously to the discussion of $\varepsilon_{K}$ and $D^{0}-\bar{D}^{0}$ mixing, the constraints on the operators $\mathcal{O}_{\text {quqd }}^{(1,8)}$ from $d_{n}$ can be illustrated by writing $d_{n}$ as a linear combination of Wilson coefficients at $\mu_{\mathrm{ew}}$,

$\frac{d_{n}}{d_{n}^{\lim }} \approx \sum_{i} \sigma_{i} \Lambda_{i}^{2} \operatorname{Im}\left[\mathcal{C}_{\text {quqd }}^{(1,8)}\right]_{i}$,

where $i$ stands for a 4-tuple of flavour indices and $d_{n}^{\lim }$ is the current $90 \%$ confidence-level upper bound on the neutron EDM [92],

$d_{n}^{\lim }=3 \times 10^{-26} e \mathrm{~cm} \approx 4.6 \times 10^{-13} \mathrm{GeV}^{-1}$.

In Fig. 3, we show the values of $\Lambda_{i}$ for the neutron EDM (obtained with flavio [59] and wilson [58]) as well as for $\varepsilon^{\prime} / \varepsilon$, parametrized analogously as

$\frac{\left(\varepsilon^{\prime} / \varepsilon\right)_{\mathrm{BSM}}}{10^{-3}} \approx \sum_{i} \sigma_{i} \Lambda_{i}^{2} \operatorname{Im}\left[\mathcal{C}_{\text {quqd }}^{(1,8)}\right]_{i}$

The chart shows that several of the operators would lead to an excessive contribution to $d_{n}$ when leading to a visible effect in $\varepsilon^{\prime} / \varepsilon$; some of them do not contribute to $\varepsilon^{\prime} / \varepsilon$; and yet others can generate $\varepsilon^{\prime} / \varepsilon$ without being constrained by $d_{n}$. We have omitted the operators that do not contribute to either of the observables.

We stress again that the correlation discussed here arises simply from CKM rotations when moving between the mass bases of up and down quarks and we have considered SMEFT that do not generate a visible effect in either observable have been omitted. The scales corresponding to $\mathcal{C}_{\text {quqd }}^{(1)}$ are shown with a lighter shading than $\mathcal{C}_{\text {quqd }}^{(8)}$ and are always higher

Wilson coefficients at $\mu_{\mathrm{ew}}$. When considering the coefficients at a high scale $\mu_{\Lambda}$, there are also RG effects in SMEFT that induce mixing between $\mathcal{O}_{\text {quqd }}^{(1,8)}$ with different flavour indices that can lead to additional dangerous contributions to $d_{n}$. Whether a visible NP effect in $\varepsilon^{\prime} / \varepsilon$ generated by any of the operators $\mathcal{O}_{\text {quqd }}^{(1,8)}$ is viable in view of the EDM constraint has to be checked carefully in specific NP models taking into account both effects.

We finally note that beyond the neutron EDM, also the EDMs of diamagnetic atoms are sensitive to $\mathrm{CP}$ violation in dipole operators and four-quark operators, in addition to leptonic and semi-leptonic CP violation. In principle a global analysis of the various EDM measurements to disentangle the different short-distance sources of $\mathrm{CP}$ violation would be useful, but currently suffers from many unknown longdistance contributions, see [93] for a recent review.

$3.5 K \rightarrow \pi \nu \bar{\nu}$ and $K \rightarrow \pi \ell^{+} \ell^{-}$

In specific NP models one often finds correlations between BSM contributions to $\varepsilon^{\prime} / \varepsilon$ and rare kaon decays, in particular with $K^{+} \rightarrow \pi^{+} \nu \bar{v}$ and $K_{L} \rightarrow \pi^{0} \nu \bar{\nu}$. In fact in all papers that addressed the $\varepsilon^{\prime} / \varepsilon$ anomaly listed in the introduction [22-40] such correlations have been investigated. Such correlations will play an important role in distinguishing various models when the theoretical status of $\varepsilon^{\prime} / \varepsilon$ improves and the branching ratios for rare kaon decays will be well measured. 

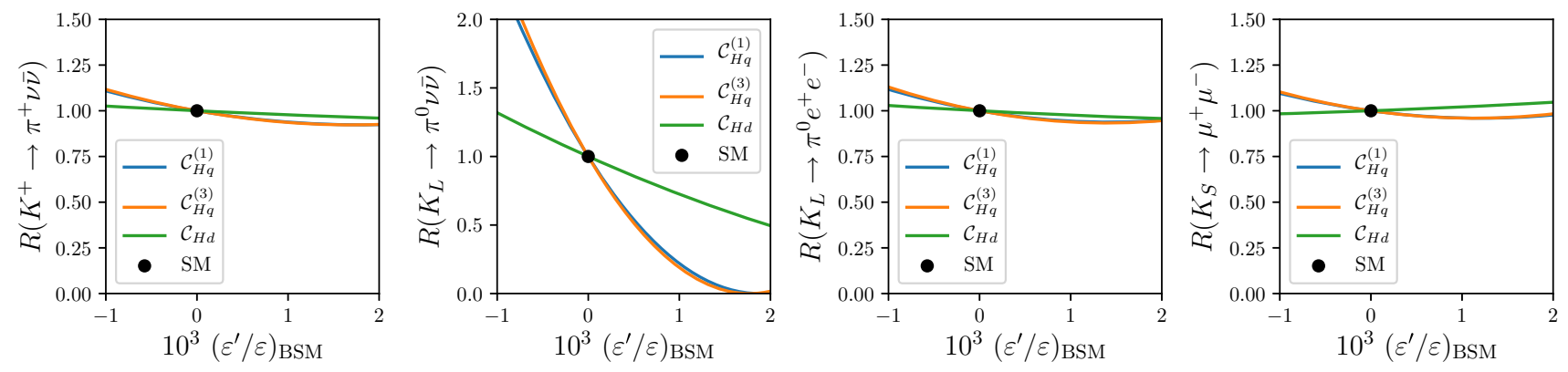

Fig. 4 Correlation between $\varepsilon^{\prime} / \varepsilon, K^{+} \rightarrow \pi^{+} \nu \bar{v}, K_{L} \rightarrow \pi^{0} v \bar{\nu}, K_{L} \rightarrow \pi^{0} e \bar{e}$, and $K_{S} \rightarrow \mu^{+} \mu^{-}$from imaginary NP effects in individual SMEFT operators of type $\psi^{2} H^{2} D$ inducing flavour-changing $Z^{0}$ couplings. The flavour index " 12 " has been suppressed

Here we would like to confine our discussion to possible model-independent correlations within a pure EFT analysis. Correlations between $\varepsilon^{\prime} / \varepsilon$ and semi-leptonic decays can then in principle arise in three different ways,

- modified $Z^{0}$ or $W^{ \pm}$couplings contributing to $\varepsilon^{\prime} / \varepsilon$ and neutral or charged current semi-leptonic decays, respectively,

- semi-leptonic operators that contribute directly to semileptonic decays and mix into $\Delta S=1$ four-quark operators by QED or electroweak RG effects, thereby contributing indirectly to $\varepsilon^{\prime} / \varepsilon$,

- four-quark operators mixing into semi-leptonic operators by QED or electroweak RG effects and contributing directly to $\varepsilon^{\prime} / \varepsilon$.

The latter two effects are strongly suppressed by the smallness of the electroweak gauge couplings; consequently $\varepsilon^{\prime} / \varepsilon$ typically dominates constraints on $\mathrm{CP}$ violation in four-quark operators, while semi-leptonic decays dominate constraints on semi-leptonic operators.

Relevant model-independent correlations could thus arise from the modified $Z^{0}$ or $W^{ \pm}$couplings induced by the SMEFT operators of type $\psi^{2} H^{2} D$ discussed in Sect. E.2. From the discussion in that section, it was concluded that imaginary parts of the following SMEFT Wilson coefficients at $\mu_{\text {ew }}$ can lead to effects in $\varepsilon^{\prime} / \varepsilon$,

$$
\begin{aligned}
& {\left[\mathcal{C}_{H d}\right]_{12}, \quad\left[\mathcal{C}_{H q}^{(1)}\right]_{12}, \quad\left[\mathcal{C}_{H q}^{(3)}\right]_{12},} \\
& {\left[\mathcal{C}_{H q}^{(3)}\right]_{13}, \quad\left[\mathcal{C}_{H q}^{(3)}\right]_{23},} \\
& {\left[\mathcal{C}_{H u d}\right]_{12}, \quad\left[\mathcal{C}_{H u d}\right]_{11} .}
\end{aligned}
$$

The coefficients of right-handed $W^{ \pm}$couplings in (44) contribute at tree-level only to charged-current semi-leptonic decays like $K \rightarrow \ell \nu_{\ell}, K \rightarrow \pi \ell \nu_{\ell}$, and beta decays (see e.g. [94-96]) and the effects in $\varepsilon^{\prime} / \varepsilon$ are essentially unconstrained at present.

The coefficients in (43), which contribute to $\varepsilon^{\prime} / \varepsilon$ only via modified left-handed $W^{ \pm}$couplings, contribute also to FCNC $B$ decays via modified $Z^{0}$ couplings. Barring unre- alistic cancellations, visible effects in $\varepsilon^{\prime} / \varepsilon$ induced by these couplings are excluded since they would lead to excessive effects e.g. in the decays $B_{S} \rightarrow \mu^{+} \mu^{-}$and $B^{0} \rightarrow \mu^{+} \mu^{-}$.

The coefficients in (42) contribute to the FCNC kaon decays of type $K \rightarrow \pi \nu \bar{v}$ and $K \rightarrow \pi \ell^{+} \ell^{-}$. These decays are sensitive to a single linear combination, namely

$\left[\mathcal{C}_{H q}^{(3)}\right]_{12}+\left[\mathcal{C}_{H q}^{(1)}\right]_{12}+\left[\mathcal{C}_{H d}\right]_{12}$,

while the leptonic FCNC decays of type $K \rightarrow \ell^{+} \ell^{-}$are sensitive to

$\left[\mathcal{C}_{H q}^{(3)}\right]_{12}+\left[\mathcal{C}_{H q}^{(1)}\right]_{12}-\left[\mathcal{C}_{H d}\right]_{12}$.

Inspecting our master formula and matching conditions, $\varepsilon^{\prime} / \varepsilon$ is instead sensitive approximately to the imaginary part of the linear combination

$\left[\mathcal{C}_{H q}^{(3)}\right]_{12}+1.1\left[\mathcal{C}_{H q}^{(1)}\right]_{12}+3.7\left[\mathcal{C}_{H d}\right]_{12}$

of these three Wilson coefficients at $\mu_{\text {ew }}$. Numerically, it turns out that a purely $\mathrm{CP}$ violating contribution to any of these three coefficients that would lead to a visible effect in $\varepsilon^{\prime} / \varepsilon$ only leads to a very small modification of the $K^{+} \rightarrow \pi^{+} \nu \bar{v}$, $K_{L} \rightarrow \pi^{0} \ell \bar{\ell}$, and $K_{S} \rightarrow \ell^{+} \ell^{-}$branching ratios, as demonstrated in Fig. 4 (see also $[24,26]$ ). In the CP violating decay $K_{L} \rightarrow \pi^{0} v \bar{v}$, a NP effect in $\varepsilon^{\prime} / \varepsilon$ in the ballpark of $10^{-3}$ would instead lead to a suppressed branching ratio. Seeing such suppression would however require an experimental sensitivity better than the SM branching ratio, which is at the level of $3 \times 10^{-11}$, still two orders of magnitude away from the recent preliminary bound from the KOTO collaboration [97],

$\operatorname{BR}\left(K_{L} \rightarrow \pi^{0} v \bar{v}\right)<3.0 \times 10^{-9} @ 90 \%$ C.L.

We conclude that $\mathrm{CP}$-violating new physics in the operators with modified $Z^{0}$ couplings in (42) or right-handed $W^{ \pm}$ couplings in (44) can lead to sizable effects in $\varepsilon^{\prime} / \varepsilon$ without appreciable constraints from semi-leptonic kaon decays. 


\section{$3.6 \Delta C=1$}

Eventually we mention that similarly to $\Delta C=2$ processes, also $\mathrm{CP}$ violation in $\Delta C=1$ decays is correlated to $\varepsilon^{\prime} / \varepsilon$ in SMEFT. The interesting observables are CP asymmetries in Cabbibo-favoured (CF) and singly-Cabbibo suppressed (SCS) $D \rightarrow M_{1} M_{2}$ decays. They are governed by the $\Delta C=1 \mathrm{EFT}$

$\mathcal{H}_{\triangle C=1}=-\sum_{i} C_{i} O_{i}$

with operators

$O_{X A B}^{q q^{\prime}}=\left(\bar{u}^{i} \Gamma_{X} P_{A} c^{i}\right)\left(\bar{q}^{j} \Gamma_{X} P_{B} q^{\prime j}\right)$,

$\widetilde{O}_{X A B}^{q q^{\prime}}=\left(\bar{u}^{i} \Gamma_{X} P_{A} c^{j}\right)\left(\bar{q}^{j} \Gamma_{X} P_{B} q^{\prime i}\right)$,

with $q q^{\prime}=s d$ (CF) and $q q^{\prime}=d d$, ss or $q q^{\prime}=u u$ (SCS). The correlations enter then via the SMEFT four-quark operators

$\mathcal{O}_{q q}^{(1)}, \mathcal{O}_{q q}^{(3)}, \quad \mathcal{O}_{q u}^{(1)}, \mathcal{O}_{q u}^{(8)}, \quad \mathcal{O}_{q d}^{(1)}, \mathcal{O}_{q d}^{(8)}, \quad \mathcal{O}_{q u q d}^{(1)}, \mathcal{O}_{q u q d}^{(8)}$,

as well as modified $Z^{0}$ and $W^{ \pm}$couplings.

The correlation of $\varepsilon^{\prime} / \varepsilon$ and $\mathrm{CP}$ asymmetries in CF decays $D^{0} \rightarrow K^{-} \pi^{+}, D_{s}^{+} \rightarrow \eta \pi^{+}$and $D_{s}^{+} \rightarrow \eta^{\prime} \pi^{+}$has been discussed [38] in the framework of a $S U(2)_{L} \otimes S U(2)_{R} \otimes$ $U(1)_{B-L}$ symmetric model. The correlation with CP asymmetries in the SCS decays $D^{0} \rightarrow K^{+} K^{-}$and $D^{0} \rightarrow \pi^{+} \pi^{-}$ has been discussed in a general EFT framework in [98]. The rich potential to distinguish among various BSM scenarios with the help of these correlations is hampered by the lack of knowledge of hadronic matrix elements in non-leptonic charm decays and we will therefore not investigate this subject further.

\section{Implications for model building}

Having discussed the general model-independent anatomy of $\varepsilon^{\prime} / \varepsilon$ below the electroweak scale and the consequences of $S U(2)_{L} \otimes U(1)_{Y}$ gauge invariance within SMEFT, we are now in a position to discuss the implications for the possible effects in BSM scenarios with new sources of CP violation where BSM effects in $\varepsilon^{\prime} / \varepsilon$ are encoded in the imaginary part of Wilson coefficients of dimension-six SMEFT operators.

The size of the coefficients $P_{i}$ in our master formula presented in Sect. 2.3, together with the matching conditions in Sect. 2.4, already indicate which scenarios are more promising than others to explain a deviation from the SM in $\varepsilon^{\prime} / \varepsilon$. However, in a concrete BSM scenario, the Wilson coefficients with the highest values of $P_{i}$ could vanish or be suppressed by small couplings. Consequently without additional dynamical assumptions or specific models no clear-cut conclusions can be made. While a comprehensive discussion of models is beyond the scope of this paper, in the following subsections we will discuss a number of general implications on the basis of simplified models with a single tree-level mediator.

A generic challenge in explaining sizable NP effects in $\varepsilon^{\prime} / \varepsilon$ is to avoid the constraint from $\varepsilon_{K}$. Roughly speaking, the $\Delta S=1 \mathrm{CP}$-odd observable $\varepsilon^{\prime} / \varepsilon$ typically probes the quantity $\operatorname{Im} \delta / \mu_{\Lambda}{ }^{2}$, where $\delta$ is a flavour-violating parameter, while the $\Delta S=2$ observable $\varepsilon_{K}$ typically probes $\operatorname{Im} \delta^{2} / \mu_{\Lambda}{ }^{2}$ (cf. [31]). Given the strong constraints from $\varepsilon_{K}$ (see Table 2), barring a tuning of the phase of $\delta$ or fine-tuned cancellations, a visible effect in $\varepsilon^{\prime} / \varepsilon$ then seemingly requires very low NP scales $\mu_{\Lambda} \lesssim 1 \mathrm{TeV}$. In the literature, this problem has been avoided in four different ways,

- through contributions from chromo-magnetic dipole operators to $\varepsilon^{\prime} / \varepsilon$ that do not affect $\varepsilon_{K}$ [36],

- through contributions from modified $Z^{0}$ couplings to $\varepsilon^{\prime} / \varepsilon$ [25-27] that only enter $\varepsilon_{K}$ through top-quark Yukawa RG effects [24],

- through contributions from modified right-handed $W^{ \pm}$ couplings to $\varepsilon^{\prime} / \varepsilon$ that do not affect $\varepsilon_{K}$ [85],

- through loop-induced contributions to $\varepsilon^{\prime} / \varepsilon$ in conjunction with an accidental suppression of the contributions to $\varepsilon_{K}$ arising in models with Majorana fermions like the MSSM [31].

In Sect. 4.2, we will present a new solution: tree-level scalar exchange can mediate $\Delta S=1$ transitions at tree level without generating $\Delta S=2$, since a dimension six operator of the form $(\bar{q} d)^{2}$ is not allowed by hypercharge invariance.

We start by listing all the possible tree-level models in Sect. 4.1. After discussing the scalar scenario in Sect. 4.2, we will comment on the challenges of models with vector mediators in Sect. 4.3 and discuss the generation of modified $Z^{0}$ and $W^{ \pm}$couplings in Sect. 4.4.

\subsection{Tree-level mediators}

The simplest models giving rise to a NP contribution to $\varepsilon^{\prime} / \varepsilon$ are models with a single tree-level mediator generating a four-quark operator. Given the large scales probed by $\varepsilon^{\prime} / \varepsilon$, clearly also models without tree-level FCNCs can give a sizable contribution to $\varepsilon^{\prime} / \varepsilon$. Nevertheless, the tree-level models can serve as benchmark cases exhibiting generic features of larger classes of models.

In Table 3, we list all the possible tree-level mediators that can generate any of the four-quark operators that give a matching contribution to $\Delta S=1$ at $\mu_{\mathrm{ew}}$ [99]. We have omitted states that permit baryon number violating cou- 
Table 3 Four-quark SMEFT operators containing down-type quarks generated by the exchange of scalar or vector mediators at tree level. The second column gives the representation under $G_{\mathrm{SM}}=S U(3)_{c} \otimes S U(2)_{L} \otimes U(1)_{Y}$

\begin{tabular}{|c|c|c|c|c|c|c|c|c|c|c|c|c|}
\hline Spin & Rep. & $\mathcal{O}_{q q}^{(1)}$ & $\mathcal{O}_{q q}^{(3)}$ & $\mathcal{O}_{q u}^{(1)}$ & $\mathcal{O}_{q u}^{(8)}$ & $\mathcal{O}_{q d}^{(1)}$ & $\mathcal{O}_{q d}^{(8)}$ & $\mathcal{O}_{u d}^{(1)}$ & $\mathcal{O}_{u d}^{(8)}$ & $\mathcal{O}_{d d}$ & $\mathcal{O}_{q u q d}^{(1)}$ & $\mathcal{O}_{q u q d}^{(8)}$ \\
\hline \multirow[t]{2}{*}{0} & $(1,2)_{\frac{1}{2}}$ & & & $x$ & $x$ & $x$ & $x$ & & & & $x$ & \\
\hline & $(8,2)_{\frac{1}{2}}$ & & & $x$ & $x$ & $x$ & $x$ & & & & & $\times$ \\
\hline \multirow[t]{6}{*}{1} & $(1,1)_{0}$ & $x$ & & $x$ & & $\times$ & & $x$ & & $\times$ & & \\
\hline & $(1,1)_{1}$ & & & & & & & $x$ & $\times$ & & & \\
\hline & $(8,1)_{0}$ & $\times$ & $\times$ & & $x$ & & $\times$ & & $x$ & $x$ & & \\
\hline & $(8,1)_{1}$ & & & & & & & $x$ & $\times$ & & & \\
\hline & $(1,3)_{0}$ & & $\times$ & & & & & & & & & \\
\hline & $(8,3)_{0}$ & $x$ & $x$ & & & & & & & & & \\
\hline
\end{tabular}

plings. ${ }^{14}$ These states are either $S U(3)_{c}$ triplets (leptoquarks) or sextets (diquarks), and the former are popular scenarios to explain current anomalies in semi-leptonic $B$ decays. Further we omitted the possibility of a heavy vector doublet $(1,2)_{\frac{1}{2}}$. For a scalar mediator, the SM gauge quantum numbers then only allow two possible representations: a heavy Higgs-like doublet under $S U(2)_{L}$ that is either a singlet or an octet under $S U(3)_{c}$. For a vector mediator, there are six possibilities, $S U(3)_{c}$ singlets or octets that are $S U(2)_{L}$ singlets or triplets.

Further tree-level contributions to $\varepsilon^{\prime} / \varepsilon$ can arise from models inducing modified $W^{ \pm}$or $Z^{0}$ couplings and will be discussed in Sect. 4.4.

\subsection{Scalar operators from scalar mediators}

The novel feature after the calculation of hadronic matrix elements of BSM operators in [43] is the importance of scalar and tensor four-quark operators. As indicated in Fig. 1 and shown in Sect. 2.4, these matrix elements are relevant in scenarios that generate the SMEFT operators $\mathcal{O}_{q u q d}^{(1,8)}$ at the electroweak scale. Table 3 shows that these operators can be mediated at tree level by heavy Higgs doublets, either a colour-singlet or a colour-octet Higgs.

Focusing on the colour-octet case (and thereby avoiding discussions of a modified SM Higgs potential), the Lagrangian necessary to generate the $\mathcal{O}_{\text {quqd }}^{(1,8)}$ operators can be written as

$\mathcal{L}=-X_{d}^{i j} \bar{q}_{i} T^{A} d_{j} \Phi^{A}-X_{u}^{i j} \bar{q}_{i} T^{A} u_{j} \tilde{\Phi}^{A}+$ h.c.

Integrating out the heavy scalar leads to the following treelevel matching conditions for the four-quark SMEFT opera-

\footnotetext{
$\overline{14}$ Even though not all of them mediate proton decay at tree level, see e.g. $[100,101]$.
}

tors at the matching scale $\mu_{\Lambda}[99]$

$$
\begin{aligned}
{\left[\mathcal{C}_{q u(q d)}^{(1)}\right]_{i j k l} } & =-\frac{4}{3}\left[\mathcal{C}_{q u(q d)}^{(8)}\right]_{i j k l}=-\frac{2}{9} \frac{X_{u(d)}^{j k *} X_{u(d)}^{i l}}{M_{\phi}^{2}} \\
{\left[\mathcal{C}_{q u q d}^{(8)}\right]_{i j k l} } & =\frac{X_{u}^{i j} X_{d}^{k l}}{M_{\phi}^{2}}
\end{aligned}
$$

Importantly, to generate $\mathcal{C}_{q u q d}^{(8)}$, the presence of both Yukawa-like couplings $X_{u}$ and $X_{d}$ is necessary. The model can thus contribute to $\varepsilon^{\prime} / \varepsilon$ both through the left-right vector operators $\mathcal{O}_{q u, q d}^{(1,8)}$ and through the scalar operators; which one is more relevant depends on the hierarchies of the CPviolating couplings.

Some of the operators in (53) are also constrained by the $\Delta F=2$ or $\Delta F=0$ processes discussed in Sect. 3. In the basis where the down-type quark mass matrix is diagonal, $\varepsilon_{K}$ is sensitive to $\left[\mathcal{C}_{q d}^{(1)}\right]_{2121}$. As seen from (53), this Wilson coefficient is proportional to $X_{d}^{12 *} X_{d}^{21}$. Interestingly, this means that an imaginary part in one of the couplings $X_{d}^{12}$ or $X_{d}^{21}$ is not constrained by $\varepsilon_{K}$ at all, but could well generate a visible effect in $\varepsilon^{\prime} / \varepsilon$. Similar comments apply to the $\Delta C=2$ constraint on flavour off-diagonal couplings in $X_{u}$.

Since the operators of type $\mathcal{O}_{\text {quqd }}^{(1,8)}$ can be generated, in models with scalar mediators also the neutron EDM, induced at the one-loop level as discussed in Sect. 3.4, can be a relevant constraint.

We leave a detailed analysis of the interesting scalar scenarios to the future.

\subsection{Models with vector mediators}

As shown in Sect. 3.3, the operators $\mathcal{O}_{q q}^{(1,3)}$ are strongly constrained by $\mathrm{CP}$ violation in $K^{0}-\bar{K}^{0}$ and $D^{0}-\bar{D}^{0}$ mixing, precluding any visible effect in $\varepsilon^{\prime} / \varepsilon$, barring unrealistic cancellations that are not stable under RG evolution. Consequently, models with a heavy mediator that only couples to 
Table 4 SMEFT operators of type $\psi^{2} H^{2} D$ inducing corrections to $W^{ \pm}$and $Z^{0}$ couplings, generated by the tree-level mixing of SM fields with heavy vector-like quarks or vector fields. The second column gives the representation under $G_{\mathrm{SM}}=S U(3)_{c} \otimes S U(2)_{L} \otimes U(1)_{Y}$

\begin{tabular}{|c|c|c|c|c|c|}
\hline Spin & Rep. & $\mathcal{O}_{H q}^{(1)}$ & $\mathcal{O}_{H q}^{(3)}$ & $\mathcal{O}_{H d}$ & $\mathcal{O}_{H u d}$ \\
\hline \multirow[t]{6}{*}{$\frac{1}{2}$} & $(3,1)_{\frac{2}{3}}$ & $x$ & $x$ & & \\
\hline & $(3,1)_{-\frac{1}{3}}$ & $x$ & $\times$ & & \\
\hline & $(3,3)_{-\frac{1}{3}}$ & $x$ & $x$ & & \\
\hline & $(3,3)_{\frac{2}{3}}$ & $x$ & $x$ & & \\
\hline & $(3,2)_{\frac{1}{6}}$ & & & $\times$ & $x$ \\
\hline & $(3,2)-\frac{5}{6}$ & & & $\times$ & \\
\hline \multirow[t]{3}{*}{1} & $(1,1)_{0}$ & $x$ & & $x$ & \\
\hline & $(1,1)_{1}$ & & & & $x$ \\
\hline & $(1,3)_{0}$ & & $\times$ & & \\
\hline
\end{tabular}

left-handed quark doublets are not among the prime candidates to explain a possible deviation from the $\mathrm{SM}$ in $\varepsilon^{\prime} / \varepsilon$.

In view of these constraints, the most attractive scenarios in the case of a tree-level vector mediator are those that can generate the left-right operators $\mathcal{O}_{q u, q d}^{(1,8)}$. This is even more so given that these operators eventually contribute to $\varepsilon^{\prime} / \varepsilon$ via matrix elements that are chirally enhanced. As seen from Table 3, the only possibilities in this case are a SM singlet $Z^{\prime}$ or a heavy gluon $G^{\prime}$, that have already been explored in the literature (see e.g. [102]), described schematically by the following Lagrangian for $Z^{\prime}$

$\mathcal{L}_{Z^{\prime}}=\left[\lambda_{q}^{i j}\left(\bar{q}_{i} \gamma_{\mu} q_{j}\right)+\lambda_{u}^{i j}\left(\bar{u}_{i} \gamma_{\mu} u_{j}\right)+\lambda_{d}^{i j}\left(\bar{d}_{i} \gamma_{\mu} d_{j}\right)\right] Z^{\prime \mu}$,

and analogously for $G^{\prime}$

$$
\begin{aligned}
\mathcal{L}_{G^{\prime}}= & {\left[\lambda_{q}^{i j}\left(\bar{q}_{i} \gamma^{\mu} T^{A} q_{j}\right)+\lambda_{u}^{i j}\left(\bar{u}_{i} \gamma^{\mu} T^{A} u_{j}\right)\right.} \\
& \left.+\lambda_{d}^{i j}\left(\bar{d}_{i} \gamma^{\mu} T^{A} d_{j}\right)\right] G_{\mu}^{\prime A} .
\end{aligned}
$$

In the case of the operators $\mathcal{O}_{q u}^{(1,8)}$, only two flavour index combinations ${ }^{15}$ contribute to the $\Delta S=1$ matching at $\mu_{\mathrm{ew}}$, namely $\left[\mathcal{O}_{q u}^{(1,8)}\right]_{1211}$ and $\left[\mathcal{O}_{q u}^{(1,8)}\right]_{1222}$. Neglecting SMEFT RG effects, this corresponds to a product of one of the real-valued couplings $\lambda_{u}^{11}$ or $\lambda_{u}^{22}$ and the complex-valued coupling $\lambda_{q}^{12}$. The square of the latter coupling also generates a contribution to $\varepsilon_{K}$. Barring a fine-tuning of the phase to $\pi / 2$, this requires $\left|\lambda_{q}^{12}\right| / M_{Z^{\prime}}$ to be below $(13 \mathrm{PeV})^{-1}$, as seen from Table 2. A visible effect in $\varepsilon^{\prime} / \varepsilon$ is then only possible for a coupling $\left|\lambda_{u}^{11}\right| / M_{Z^{\prime}}$ not smaller than $(10 \mathrm{TeV})^{-1}$. For masses within reach of the $\mathrm{LHC}$, this implies a large cross section $p p \rightarrow j j$, and the $p p \rightarrow j j$ angular distribution allows to constrain operators with flavour structure $(\bar{u} u)(\bar{u} u)$ even beyond resonance production. Whether such a model remains

\footnotetext{
15 We again omit redundant operators.
}

viable in view of these stringent bounds deserves a dedicated study.

In the case of the operators $\mathcal{O}_{q d}^{(1,8)}$, more flavour index combinations contribute to the $\Delta S=1$ matching at $\mu_{\mathrm{ew}}$ as seen in Sect. 2.4, since they can also contribute via righthanded down-type quarks and left-handed up-type quarks. Nevertheless, a contribution to $\varepsilon_{K}$ is generated either by the 12-coupling ${ }^{16}$ to right-handed or to left-handed down-type quarks. Consequently, comparably stringent bounds as in the case of $\mathcal{O}_{q u}^{(1,8)}$ apply.

We finally note that models where a vector mediator dominantly contributes to $\varepsilon^{\prime} / \varepsilon$ via the purely right-handed fourquark operators $\mathcal{O}_{u d}^{(1,8)}$ or $\mathcal{O}_{d d}$ are subject to similar constraints from $\Delta F=2$ and dijets, but their contributions to $\varepsilon^{\prime} / \varepsilon$ are not chirally enhanced, as shown in Sect. 2.3, such that a sizable contribution to $\varepsilon^{\prime} / \varepsilon$ is even more difficult to attain.

\subsection{Models with modified electroweak couplings}

Apart from a tree-level exchange of heavy scalar or vector bosons, $\varepsilon^{\prime} / \varepsilon$ can also arise at tree level in the SMEFT from the operators of type $\psi^{2} H^{2} D$ that induce modified couplings to the $Z^{0}$ and $W^{ \pm}$bosons. In the broken phase of the SM, these contributions can be seen as arising from the mixing between SM fermion or boson fields with heavy vector-like fermions or vector bosons after electroweak symmetry breaking. In Table 4, we list all the possible vector-like fermion or vector boson representations that generate any of the $\psi^{2} H^{2} D$ operators that give a matching contribution to $\Delta S=1$ at $\mu_{\mathrm{ew}}$ [99].

The vector-like fermion representations have already been discussed in detail in the context of $\varepsilon^{\prime} / \varepsilon$ in [26], with

\footnotetext{
16 The only way to generate a $\Delta S=1$ operator at $\mu_{\mathrm{ew}}$ without a 12-coupling is via the operators $\left[\mathcal{O}_{q d}^{(1,8)}\right]_{1332}$; however, they match onto $C_{S L R}^{b}$ and $\widetilde{C}_{S L R}^{b}$, which contribute to $\varepsilon^{\prime} / \varepsilon$ neither directly nor indirectly, as shown in Sect. 2.2.
} 
the exception of the state $(3,1)_{2 / 3}$ that transforms like a right-handed up-type quark singlet. In this case, one gets $C_{H q}^{(1)}=-C_{H q}^{(3)}$, such that there is no flavour-changing $Z^{0}$ coupling and thus no contribution to semi-leptonic FCNCs (cf. Sect. 3.5), but a contribution to $\varepsilon^{\prime} / \varepsilon$ can nevertheless arise from a modified left-handed $W^{ \pm}$coupling.

The three spin- 1 models in Table 4 already appeared in Table 3; these states can contribute both through tree-level exchange leading to a four-quark SMEFT operator or through modified $W^{ \pm}$or $Z^{0}$ couplings. Which contribution dominates depends on the size of the couplings. Given the strong constraints from $\varepsilon_{K}$ on contributions from four-quark operators in models with vector mediators discussed in Sect. 4.3, it is an interesting question how important this constraint is when $\varepsilon^{\prime} / \varepsilon$ is dominantly generated through flavour-changing $Z^{0}$ couplings. In the $(1,1)_{0}$ model, i.e. with a SM singlet $Z^{\prime}$, there are two relevant couplings for this discussion [99],

$\mathcal{L} \supset\left[\lambda_{q}^{21}\left(\bar{q}_{2} \gamma_{\mu} q_{1}\right)+\lambda_{H}\left(H^{\dagger} i D_{\mu} H\right)\right] Z^{\prime \mu}+$ h.c.

Rescaling the couplings as $\Delta_{i} \equiv \lambda_{i} / m_{Z^{\prime}}$, the Wilson coefficients relevant for $\Delta S=1$ and $\Delta S=2 \mathrm{read}$ [99]

$\left[\mathcal{C}_{H q}^{(1)}\right]_{12}=-\Delta_{q} \operatorname{Re} \Delta_{H}, \quad\left[\mathcal{C}_{q q}^{(1)}\right]_{1212}=-\frac{1}{2} \Delta_{q}^{2}$.

In addition, a contribution to the Wilson coefficient of the purely bosonic operator $\mathcal{O}_{H D}$ is generated,

$\mathcal{C}_{H D}=-2\left(\operatorname{Re} \Delta_{H}\right)^{2}$.

This Wilson coefficient is related to the electroweak $T$ parameter as

$T=-2 \pi v^{2} \frac{g^{2}+g^{\prime 2}}{g^{2} g^{\prime 2}} \mathcal{C}_{H D}$.

This allows to write the magnitude of the BSM effect in $\varepsilon^{\prime} / \varepsilon$ induced by $\mathcal{C}_{H q}^{(1)}$ in terms of the shifts in $\varepsilon_{K}$ and the $T$ parameter as

$10^{3}\left|\frac{\varepsilon^{\prime}}{\varepsilon}\right|_{\mathrm{BSM}} \approx 0.1\left|\left[\frac{\left(\varepsilon_{K}\right)_{\mathrm{BSM}}}{10^{-3}}\right]\left[\frac{T_{\mathrm{BSM}}}{0.1}\right]\left[\frac{\operatorname{Im} \Delta_{q}}{\operatorname{Re} \Delta_{q}}\right]\right|^{\frac{1}{2}}$.

Given that the measurement of $\varepsilon_{K}$ agrees with the SM at the level of $0.5 \times 10^{-3}$ and the $T$ parameter at the level of 0.05 , barring cancellations, this shows that the $Z^{0}$-mediated effect is strongly constrained unless the phase of $\Delta_{q}$ is tuned close to $\pi / 2$.

For the vector triplet $(1,3)_{0}$, the analogous contribution to the $T$ parameter is absent, so the $Z^{0}$-mediated contribution to $\varepsilon^{\prime} / \varepsilon$ could be sizable.

The $S U(2)_{L}$ singlet charged gauge boson $(1,1)_{1}$ could arise as the low-energy limit of a broken left-right symmetry (see e.g. [103]). In this case, the contribution to $\varepsilon^{\prime} / \varepsilon$ is mediated by a right-handed $W^{ \pm}$coupling, such that $\varepsilon_{K}$ gives no constraint.
4.5 Models with dipole operators

The chromomagnetic dipole operators $O_{8 g}^{(\prime)}$ can arise in various BSM scenarios. While the corresponding matrix element and thus the value of $P_{i}$ in our master formula is small, the absence of model-independent constraints on this contribution makes it nevertheless interesting.

Since the SMEFT dipole operator $\mathcal{O}_{d G}$ does not receive tree-level matching contributions, the dipole operators at the low-energy scale $\mu$ can arise either from four-quark operators mixing into it through RG evolution or from loop-induced matching contributions at the UV scale $\mu_{\Lambda}$. Concerning the former effect, in Sects. 2.2 and 2.4, we have shown that SMEFT scalar operators of type $\mathcal{O}_{\text {quqd }}^{(1,8)}$ can induce such a contribution. Whether this contribution is relevant depends on the structure of the couplings (cf. Sect. 4.2):

- If they dominantly match onto the scalar $\Delta S=1$ operators with flavour $(\bar{s} d)(\bar{u} u)$ in Class $\mathrm{C}$, these have themselves also non-vanishing $K \rightarrow \pi \pi$ matrix elements and contribute directly to $\varepsilon^{\prime} / \varepsilon$, such that the indirect contribution via the dipole operator is negligible.

- If they dominantly match onto the scalar $\Delta S=1$ operators with flavour $(\bar{s} d)(\bar{c} c)$ in Class B, they indeed contribute to $\varepsilon^{\prime} / \varepsilon$ exclusively via the dipole Wilson coefficient at the low-energy scale.

- If the scalars couple dominantly to top quarks (see e.g. [36]), these operators do not match at tree-level onto the $\Delta S=1$ EFT (where top quarks have already been integrated out), but RG evolution above $\mu_{\mathrm{ew}}$ (cf. Sect. 2.4) will generate the SMEFT dipole operator $\mathcal{O}_{d G}$.

In models with heavy scalars (but no heavy fermions), also one-loop matching contributions at the scale $\mu_{\Lambda}$ exist. However, in the SMEFT, where SM quarks are massless, these contributions are IR-divergent by themselves. The divergence is cancelled by the RG-induced contribution of the scalar four-quark operators $\mathcal{O}_{\text {quqd }}^{(1,8)}$.

In models with heavy vectors but no heavy fermions, we expect that typically four-quark operator contributions are more important than loop-induced dipole operator contributions, again with the possible exception of top quarks, where RG-induced effects above $\mu_{\text {ew }}$ are relevant.

In models with new heavy vector-like fermions that couple to the Higgs doublet, sizable contributions to the dipole operator can be generated from a diagram with a SM Higgs in the loop. This gives an important constraint in models with partial quark compositeness [54,104-107].

Finally, there can of course also be loop contributions at $\mu_{\Lambda}$ with only new heavy particles in the loop. This has been for example studied in MSSM [30-34], where scalar operators are usually omitted because they are suppressed by light-quark Yukawa couplings, although some might be $\tan \beta$ 
enhanced, whereas the one-loop contribution to the dipole operator is not suppressed.

\section{Summary}

We have presented for the first time a model-independent anatomy of the ratio $\varepsilon^{\prime} / \varepsilon$ in the context of the $\Delta S=1 \mathrm{EFT}$ with operators invariant under QCD and QED and in the context of the SMEFT with the operators invariant under the full SM gauge group. This was only possible thanks to the very recent calculations of the $K \rightarrow \pi \pi$ matrix elements of BSM operators, namely of the chromo-magnetic dipole operators by lattice QCD [41] and DQCD [42] and in particular through the calculation of matrix elements of all four-quark BSM operators, including scalar and tensor operators, by DQCD [43]. Even if the latter calculations have been performed in the chiral limit, they offer for the first time a look into the world of BSM operators contributing to $\varepsilon^{\prime} / \varepsilon$.

Our main goal was to identify those new physics scenarios which are probed by $\varepsilon^{\prime} / \varepsilon$ and which could help to explain the emerging anomaly in $\varepsilon^{\prime} / \varepsilon$, which is signalled both by lattice QCD results and results from the DQCD approach. To this end we have derived a master formula for $\varepsilon^{\prime} / \varepsilon$, presented already in [46], which can be applied to any theory beyond the SM in which the Wilson coefficients of all contributing operators have been calculated at the electroweak scale. The relevant hadronic matrix elements of BSM operators are from the DQCD approach and the SM ones from lattice QCD.

In the last three years a number of analyses, addressing the $\varepsilon^{\prime} / \varepsilon$ anomaly in concrete models, appeared in the literature (see list at the beginning of our paper) but they concentrated on models in which NP entered exclusively through modifications of the Wilson coefficients of SM operators. In particular the Wilson coefficient of the dominant electroweak penguin operator $Q_{8}$ plays an important role in this context as its hadronic matrix element is chirally enhanced and in contrast to the QCD penguin operator $Q_{6}$ this contribution is not suppressed by the factor $1 / \omega \approx 22$ related to the $\Delta I=1 / 2$ rule. While we confirm these findings through the analysis of models that generate operators of Class A, this is a significant limitation if one wants to have a general view of possible BSM scenarios responsible for the $\varepsilon^{\prime} / \varepsilon$ anomaly. In particular, in the absence of even approximate values of hadronic matrix elements of BSM operators, no complete model-independent analysis was possible until recently.

The recent calculations of BSM $K \rightarrow \pi \pi$ matrix elements, in particular of those of scalar and tensor operators in [43], combined with the EFT and in particular SMEFT analyses presented in our paper, widened significantly our view on BSM contributions to $\varepsilon^{\prime} / \varepsilon$.

Our analysis has two main virtues:
- It opens the road to the analyses of $\varepsilon^{\prime} / \varepsilon$ in any theory beyond the SM and allows with the help of the master formula in (18) [46], with details presented here, to search very efficiently for BSM scenarios behind the $\varepsilon^{\prime} / \varepsilon$ anomaly. In particular the values of $P_{i}$ collected in "Appendix C" indicate which routes are more promising than others, both in the context of the low-energy EFT and SMEFT. By implementing our results in the open source code flavio [59], testing specific BSM theories becomes particularly simple.

- Through our SMEFT analysis we were able to identify correlations between $\varepsilon^{\prime} / \varepsilon$ and various observables that depend sensitively on the operators involved. Here $\Delta S=$ 2, $\Delta C=2$ and electro-magnetic dipole moments (EDM) play a prominent role but also correlations with $\Delta S=1$ and $\Delta C=1$ provide valuable informations.

Our take-home messages are:

- Tree-level vector exchanges, like $Z^{\prime}$ and $G^{\prime}$ contributions, discussed already by various authors, can be responsible for the observed anomaly. In these scenarios one has to face in general important constraints from $\Delta S=2$ and $\Delta C=2$ transitions as well as direct searches and often some fine tuning is required. Here the main role is played by the electroweak operator $Q_{8}$ with its Wilson coefficient significantly modified by NP.

- Models with tree-level exchange of heavy colourless or coloured scalars are a new avenue, opened by the results for BSM operators from DQCD in [43]. In particular scalar and tensor operators, having chirally enhanced matrix elements and consequently large coefficients $P_{i}$, are candidates for the explanation of the anomaly in question. Moreover, some of these models, in contrast to models with tree-level $Z^{\prime}$ and $G^{\prime}$ exchanges, are free from both $\Delta S=2$ and $\Delta C=2$ constraints. The EDM of the neutron is an important constraint for these models, depending on the couplings, but does not preclude a sizable NP effect in $\varepsilon^{\prime} / \varepsilon$.

- Models with modified $W^{ \pm}$or $Z^{0}$ couplings can induce sizable effects in $\varepsilon^{\prime} / \varepsilon$ without appreciable constraints from semi-leptonic decays such as $K^{+} \rightarrow \pi^{+} \nu \bar{\nu}$ or $K_{L} \rightarrow \pi^{0} \ell \bar{\ell}$. In the case of a SM singlet $Z^{\prime}$ mixing with the $Z^{0}$, sizable $Z^{0}$-mediated contributions are disfavoured by electroweak precision tests.

The future of $\varepsilon^{\prime} / \varepsilon$ in the SM and in the context of searches for NP will depend on how accurately it can be calculated. This requires improved lattice calculations not only of the matrix elements of SM operators but also of the BSM ones, which are known presently only from the DQCD approach in the chiral limit. It is also hoped that lattice QCD will be able to take into account isospin breaking corrections and that 
other lattice collaborations will attempt to calculate hadronic matrix elements of all relevant operators. In this context we hope that the new analysis of the RBC-UKQCD collaboration with improved matrix elements to be expected this year will shed new light on the hinted anomaly. Such future updates can be easily accounted for by the supplementary details on the master formula in "Appendix C".

On the short-distance side the NNLO results for QCD penguins should be available soon [14]. The dominant NNLO corrections to electroweak penguins have been calculated almost 20 years ago [11] and, as we have pointed out, play a significant role in removing the scale uncertainty in $m_{t}(\mu)$ and the uncertainty due to renormalization scheme dependence. Moreover, as we have seen, its inclusion increases the size of the $\varepsilon^{\prime} / \varepsilon$ anomaly. With present technology a complete NNLO calculation, using the results in [12], should be feasible in a not too distant future. As far as BSM operators are concerned, a NLO analysis of their Wilson coefficients is in progress, but its importance is not as high as of hadronic matrix elements due to significant additional parametric uncertainties residing in any NP model. In any case, in the coming years the ratio $\varepsilon^{\prime} / \varepsilon$ is expected to play a significant role in the search for NP. In this respect, the results presented here will be helpful in disentangling potential models of new CP violating sources beyond the SM as well as constraining the magnitude of their effects.

Acknowledgements We would like to thank Jean-Marc Gérard for discussions and Aneesh Manohar for clarifying communications. This work was supported by the DFG cluster of excellence "Origin and Structure of the Universe".

Data Availability Statement This manuscript has associated data in a data repository. [Authors' comment: The master formula generated during the current study is implemented in the public code flavio in the repository https://github.com/flav-io/flavio.]

Open Access This article is distributed under the terms of the Creative Commons Attribution 4.0 International License (http://creativecomm ons.org/licenses/by/4.0/), which permits unrestricted use, distribution, and reproduction in any medium, provided you give appropriate credit to the original author(s) and the source, provide a link to the Creative Commons license, and indicate if changes were made. Funded by SCOAP $^{3}$.

\section{A. $\Delta S=1$ EFT operators}

In full generality, the $\Delta S=1$ dimension-six effective Hamiltonian with $N_{f}$ active quark flavours,

$\mathcal{H}_{\Delta S=1}^{\left(N_{f}\right)}=-\sum_{i} C_{i} O_{i}$,

contains three classes of operators relevant to $K \rightarrow \pi \pi$ decays: four-quark operators

$$
\begin{aligned}
& O_{X A B}^{q}=\left(\bar{s}^{i} \Gamma_{X} P_{A} d^{i}\right)\left(\bar{q}^{j} \Gamma_{X} P_{B} q^{j}\right), \\
& \widetilde{O}_{X A B}^{q}=\left(\bar{s}^{i} \Gamma_{X} P_{A} d^{j}\right)\left(\bar{q}^{j} \Gamma_{X} P_{B} q^{i}\right),
\end{aligned}
$$

electro- and chromo-magnetic dipole operators

$O_{7 \gamma}^{(\prime)}=m_{s}\left(\bar{s} \sigma^{\mu \nu} P_{L(R)} d\right) F_{\mu \nu}$,
$O_{8 g}^{(\prime)}=m_{s}\left(\bar{s} \sigma^{\mu \nu} T^{A} P_{L(R)} d\right) G_{\mu \nu}^{A}$,

semi-leptonic operators

$O_{X A B}^{\ell}=\left(\bar{s} \Gamma_{X} P_{A} d\right)\left(\bar{\ell} \Gamma_{X} P_{B} \ell\right)$.

Here $i, j$ are colour indices, $A, B=L, R$, and $X=S, V, T$ with $\Gamma_{S}=1, \Gamma_{V}=\gamma^{\mu}, \Gamma_{T}=\sigma^{\mu \nu}$. The semi-leptonic operators can contribute to $\varepsilon^{\prime} / \varepsilon$ only via QED RG mixing and we neglect them throughout. Likewise, we neglect the electromagnetic dipole operators $O_{7 \gamma}^{(\prime)}$. This is justified because the electro- and chromo-magnetic dipole operators mix under QCD and therefore UV complete models always generate both operators with a suppression of $\alpha_{e} / \alpha_{s}$ for the electromagnetic dipole operator in $\varepsilon^{\prime} / \varepsilon$ with respect to the chromomagnetic one.

The number of $\Delta S=1$ four-quark operators is sizable. For $N_{f}=5$, there are $10+10^{\prime}$ (the prime denotes the number of chirality-flipped operators) linearly independent operators for each $q=u, c, b$ :

$O_{V L L}^{q}, \quad O_{V L R}^{q}, \quad O_{S L R}^{q}, \quad O_{T L L}^{q}$,

as well as their colour-flipped $(\widetilde{O})$ and chirality-flipped $(L \leftrightarrow R)$ counterparts. For $q=d, s$, Fierz symmetry allows to eliminate half of them, leaving only $5+5^{\prime}$ linearly independent ones. As our $\Delta S=1$ reference basis we choose to eliminate $\widetilde{O}_{i}^{d, s}$ through the relations

$$
\begin{aligned}
& \widetilde{O}_{V L L}^{d, s}=O_{V L L}^{d, s}, \\
& \widetilde{O}_{V L R}^{d}=-2 O_{S R L}^{d}, \\
& \widetilde{O}_{S L R}^{d}=-\frac{1}{2} O_{V R L}^{d}, \\
& \widetilde{O}_{V L R}^{s}=-2 O_{S L R}^{s}, \\
& \widetilde{O}_{S L R}^{s}=-\frac{1}{2} O_{V L R}^{s}, \\
& \widetilde{O}_{S L L}^{d, s}=-\frac{1}{2} O_{S L L}^{d, s}-\frac{1}{8} O_{T L L}^{d, s}, \\
& \widetilde{O}_{T L L}^{d, s}=-6 O_{S L L}^{d, s}+\frac{1}{2} O_{T L L}^{d, s},
\end{aligned}
$$

and likewise for their chirality-flipped counterparts. Hence in total there are $40+40^{\prime}$ linearly independent four-quark operators in $\mathcal{H}_{\Delta S=1}^{(5)}, 30+30^{\prime}$ in $\mathcal{H}_{\Delta S=1}^{(4)}$, and $20+20^{\prime}$ in $\mathcal{H}_{\Delta S=1}^{(3)}$. 
We note that this reference basis coincides with the "flavio" basis defined in the Wilson coefficient exchange format (WCxf) [63] and used in the flavio [59] and wilson [58] packages up to two differences,

- the normalization of the operators differs,

- the operators in the "flavio" basis have the flavour structure $(\bar{d} s)$ rather than $(\bar{s} d)$.

The complete basis can be inspected on the WCxf web site [108].

\section{B. $K \rightarrow \pi \pi$ matrix elements}

The $K \rightarrow \pi \pi$ matrix elements $\left\langle O_{i}\right\rangle_{I}$, see (5), of the operators $O_{i}$ in the $\Delta S=1$ effective Hamiltonian are a crucial input to the prediction of $\varepsilon^{\prime} / \varepsilon$ in the SM and beyond. In this appendix we count the number of irreducible matrix elements (i.e. which cannot be related to other matrix elements by exact or nearly exact symmetries like parity and isospin) and relate the matrix elements in our operator basis to the traditional SM operator basis.

As discussed in Sect. 2, the $\Delta S=1$ effective Hamiltonian with three active quark flavours (4) contains 40 four-quark operators, half of which are related to the other ones by parity, leaving at most 20 irreducible matrix elements for each of the two isospin amplitudes. Since the operators with flavour content $(\bar{s} d)(\bar{s} s)$ are expected to be strongly suppressed and we neglect them, this number reduces to the 15 matrix elements

$$
\left\langle\widetilde{O}_{X L B}^{u}\right\rangle_{I}, \quad\left\langle O_{X L B}^{u}\right\rangle_{I}, \quad\left\langle O_{X L B}^{d}\right\rangle_{I},
$$

where $X L B=V L L, V L R, S L L, S L R$, or $T L L$ (note that the operators $\widetilde{O}_{X L B}^{d}$ are Fierz-redundant). In addition, isospin relations can be used to show that ${ }^{17}$

$$
\begin{aligned}
& \left\langle O_{X L B}^{u}\right\rangle_{2}+\left\langle O_{X L B}^{d}\right\rangle_{2}=0 \\
& \left\langle\widetilde{O}_{X L B}^{u}\right\rangle_{2}+\left\langle\widetilde{O}_{X L B}^{d}\right\rangle_{2}=0
\end{aligned}
$$

These 10 relations allow to remove 10 of the $15 I=2$ matrix elements. In summary, assuming strong isospin symmetry, there are in total 15 irreducible matrix elements for $I=0$ and 5 for $I=2$. Of these, 7 and 3 are relevant in the SM, respectively.

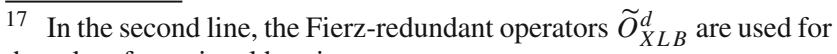
the sake of notational brevity.
}

In terms of the traditional SM operator basis [8], the matrix elements of operators in our basis can be written as Class A

$$
\begin{aligned}
\left\langle O_{V L L}^{u}\right\rangle_{I} & =\frac{1}{12}\left\langle Q_{3}\right\rangle_{I}+\frac{1}{6}\left\langle Q_{9}\right\rangle_{I}, \\
\left\langle O_{V L R}^{u}\right\rangle_{I} & =\frac{1}{12}\left\langle Q_{5}\right\rangle_{I}+\frac{1}{6}\left\langle Q_{7}\right\rangle_{I}, \\
\left\langle\widetilde{O}_{V L L}^{u}\right\rangle_{I} & =-\frac{1}{6}\left\langle Q_{3}\right\rangle_{I}+\frac{1}{6}\left\langle Q_{9}\right\rangle_{I}+\frac{1}{4}\left\langle Q_{4}\right\rangle_{I}, \\
\left\langle\widetilde{O}_{V L R}^{u}\right\rangle_{I} & =\frac{1}{12}\left\langle Q_{6}\right\rangle_{I}+\frac{1}{6}\left\langle Q_{8}\right\rangle_{I}, \\
\left\langle O_{V L L}^{d}\right\rangle_{I} & =\frac{1}{6}\left\langle Q_{3}\right\rangle_{I}-\frac{1}{6}\left\langle Q_{9}\right\rangle_{I}, \\
\left\langle O_{V L R}^{d}\right\rangle_{I} & =\frac{1}{6}\left\langle Q_{5}\right\rangle_{I}-\frac{1}{6}\left\langle Q_{7}\right\rangle_{I}, \\
\left\langle O_{S R L}^{d}\right\rangle_{I} & =-\frac{1}{12}\left\langle Q_{6}\right\rangle_{I}+\frac{1}{12}\left\langle Q_{8}\right\rangle_{I} .
\end{aligned}
$$

The isospin relations (70) in this case simply imply the vanishing of $I=2$ matrix elements of QCD penguin operators, $\left\langle Q_{3,4,5,6}\right\rangle_{2}=0$.

For the remaining 10 irreducible matrix elements, we use the results from the so-called "SD-basis" in Tables 4 and 5 of [43]. They are related to the matrix elements of operators in our basis as follows: Class $\mathbf{C}$

$$
\begin{aligned}
\left\langle O_{S L L}^{u}\right\rangle_{I}=\left\langle Q_{2}^{\mathrm{SLL}, u}\right\rangle_{I}, & \left\langle O_{T L L}^{u}\right\rangle_{I}=-\left\langle Q_{4}^{\mathrm{SLL}, u}\right\rangle_{I}, \\
\left\langle\widetilde{O}_{S L L}^{u}\right\rangle_{I}=\left\langle Q_{1}^{\mathrm{SLL}, u}\right\rangle_{I}, & \left\langle\widetilde{O}_{T L L}^{u}\right\rangle_{I}=-\left\langle Q_{3}^{\mathrm{SLL}, u}\right\rangle_{I} .
\end{aligned}
$$

\section{Class D}

$$
\left\langle O_{S L L}^{d}\right\rangle_{I}=\left\langle Q_{2}^{\mathrm{SLL}, d}\right\rangle_{I}, \quad\left\langle O_{T L L}^{d}\right\rangle_{I}=\left\langle Q_{1}^{\mathrm{SLL}, d}\right\rangle_{I} .
$$

Class E

$$
\left\langle O_{S L R}^{u}\right\rangle_{I}=\left\langle Q_{2}^{\mathrm{SLR}, u}\right\rangle_{I}, \quad\left\langle\widetilde{O}_{S L R}^{u}\right\rangle_{I}=\left\langle Q_{1}^{\mathrm{SLR}, u}\right\rangle_{I} .
$$

The isospin relations (70) allow to eliminate the $I=2$ matrix elements in (72) and (74).

In Table 5, we show the numerical values of the $K \rightarrow \pi \pi$ matrix elements of all operators entering our analysis. The ones of the SM operators are obtained from lattice QCD with $\mathrm{RG}$ evolution to $\mu=1.3 \mathrm{GeV}$ used in our numerical analysis.

\section{C. $\varepsilon^{\prime} / \varepsilon$ Master formula for new physics}

For the convenience of the reader, in this appendix we provide the details to the semi-numerical master formula (18) for the BSM contributions to $\varepsilon^{\prime} / \varepsilon$ in terms of the $\Delta S=1$ Wilson coefficients at the scale $\mu_{\mathrm{ew}}=160 \mathrm{GeV}$ and the matrix elements. We reiterate that we perform the RG evolution of NP Wilson coefficients only at the one-loop level in QCD and QED, so we do not take into account contributions that only arise at two-loop level. The numerical values of the $p_{i j}^{(I)}$ and $P_{i}$ corresponding to the five classes of operators introduced in Sect. 2.2 are listed in the following tables. 
Table 5 Numerical values of $K \rightarrow \pi \pi$ hadronic matrix elements used in our analysis. The matrix elements of the operators in the traditional SM basis $Q_{3 \ldots .}$ are based on lattice QCD $[15,16]$, the ones of the BSM operators and the chromo-magnetic dipole operator on DQCD [42,43]. All matrix elements are given in the $\overline{\mathrm{MS}}$ scheme at $\mu=1.3 \mathrm{GeV}$ and in units of $\mathrm{GeV}^{3}$. The normalization convention is chosen as $h=1$ (at variance with Refs. $[15,16])$. The values in square brackets are not needed since they can be expressed in terms of the others by isospin and Fierz relations. Note that the chromo-magnetic matrix element refers to our convention, see (6)

\begin{tabular}{llll}
\hline$Q_{i}$ & $\left\langle Q_{i}\right\rangle_{0}$ & $\left\langle Q_{i}\right\rangle_{2}$ & Refs. \\
\hline$Q_{3}$ & $-0.0399(652)(118)$ & 0 & 0 \\
$Q_{4}$ & $0.267(93)(65)$ & 0 & {$[15]$} \\
$Q_{5}$ & $-0.179(48)(46)$ & 0 & {$[15]$} \\
$Q_{6}$ & $-0.339(97)(91)$ & $0.1220(52)(71)$ & {$[15]$} \\
$Q_{7}$ & $0.155(37)(53)$ & $0.838(28)(31)$ & $0.0162(3)(6)$ \\
$Q_{8}$ & $1.54(6)(41)$ & {$[-0.003]$} & {$[15,16]$} \\
$Q_{9}$ & $-0.197(54)(49)$ & {$[-0.031]$} & {$[-0.262]$} \\
$Q_{1}^{\text {SLL }, u}$ & $-0.005(1)$ & {$[-0.151]$} & {$[15,16]$} \\
$Q_{2}^{\text {SLL }, u}$ & $-0.044(9)$ & -0.002 & {$[43]$} \\
$Q_{3}^{\text {SLL }, u}$ & $-0.371(74)$ & 0.031 \\
$Q_{4}^{\text {SLL }, u}$ & $-0.214(43)$ & {$[0.003]$} \\
$Q_{1}^{\text {SLL }, d}$ & $0.0070(14)$ & {$[0.050]$} \\
$Q_{2}^{\text {SLL }, d}$ & $-0.088(18)$ & 0 & {$[43]$} \\
$Q_{1}^{\text {SLR }, u}$ & $-0.015(3)$ & {$[43]$} \\
$Q_{2}^{\text {SLR }, u}$ & $-0.141(28)$ & {$[43]$} \\
$O_{8 g}$ & $-0.013(4)$ & {$[43]$} \\
\hline
\end{tabular}

- Table 6 contains the contributions from the Wilson coefficients from Class A that multiply SM matrix elements only.

- Table 7 contains the contributions from the Wilson coefficients from Class B that only enter via RG mixing into the chromo-magnetic dipole operator.

- Table 8 contains the contributions from the RLRL type operators of Class $\mathrm{C}$ with flavour structure $(\bar{s} d)(\bar{u} u)$ that contribute via BSM matrix elements or the chromomagnetic dipole matrix element.

- Table 9 contains the contributions from the RLRL type operators from Class D with flavour structure $(\bar{s} d)(\bar{d} d)$ that contribute via BSM matrix elements or the chromomagnetic dipole matrix element.

- Table 10 contains the contributions from the RLLR type operators from Class E with flavour structure $(\bar{s} d)(\bar{u} u)$ that contribute via matrix elements of SM operators $Q_{7,8}$ and BSM matrix elements.

Besides the $p_{i j}^{(I)}$ and $P_{i}$, we provide in the last column of each table the suppression scale $\Lambda$ that would generate $\left(\varepsilon^{\prime} / \varepsilon\right)_{\mathrm{BSM}}=10^{-3}$ for $C_{i}=1 / \Lambda^{2}$.

In these tables we restrict ourselves to listing the Wilson coefficients $C_{X A B}^{q}$ with $A=L$ since parity invariance of QED and QCD implies that the $p_{i j}^{(I)}$ are symmetric under the interchange of all $L$ and $R$. However, the $K \rightarrow \pi \pi$ matrix elements flip their sign [109]

$\left\langle(\pi \pi)_{I}\left|O_{i}\right| K\right\rangle=-\left\langle(\pi \pi)_{I}\left|\left[O_{i}\right]_{L \leftrightarrow R}\right| K\right\rangle$.

In the master formula (18), this is accounted for by the relative sign between the primed and unprimed Wilson coefficients.

The Wilson coefficients $C_{S L R}^{b, c}$ and $\widetilde{C}_{S L R}^{b, c}$ do not contribute at all at the level considered, since they do not mix at one-loop level into any of the operators with non-vanishing $K \rightarrow \pi \pi$ matrix element.

\section{SMEFT operators}

In general, the following classes of SMEFT operators can contribute to the matching onto the $\Delta S=1 \mathrm{EFT}$ at $\mu_{\mathrm{ew}}$ :

- The $\psi^{2} H X$ dipole operators $\mathcal{O}_{d B}, \mathcal{O}_{d W}, \mathcal{O}_{d G}$.

- The $\psi^{4}$ non-leptonic operators $\mathcal{O}_{d d}, \quad \mathcal{O}_{u d}^{(1,8)}$, $\mathcal{O}_{q u}^{(1,8)}, \mathcal{O}_{q d}^{(1,8)}, \mathcal{O}_{q q}^{(1,3)}, \mathcal{O}_{q u q d}^{(1,8)}$

- Contributions from modified $W^{ \pm}$and $Z^{0}$ couplings are generated by $\psi^{2} H^{2} D$ operators $\mathcal{O}_{H q}^{(1,3)}$ and $\mathcal{O}_{H d}$ that mediate both non- and semi-leptonic transitions. The $\psi^{2} H^{3}$ operator $\mathcal{O}_{d H}$ parametrizes modified $h^{0}$ couplings and contributes via tree-level $h^{0}$ exchange, but for light quark- and lepton-Yukawa couplings such exchange counts as a dimension-eight contribution [65]. 
Table 6 Coefficients $p_{i j}^{(I)}$ and $P_{i}$ of the master formula (18)-(19) as well as suppression scale $\Lambda$ (last column) from Wilson coefficients of operators in Class A multiplying SM matrix elements only. Empty entries correspond to coefficients $<0.05$

\begin{tabular}{|c|c|c|c|c|c|c|c|c|c|c|c|c|c|}
\hline & $\left\langle Q_{3}\right\rangle_{0}$ & $\left\langle Q_{4}\right\rangle_{0}$ & $\left\langle Q_{5}\right\rangle_{0}$ & $\left\langle Q_{6}\right\rangle_{0}$ & $\left\langle Q_{7}\right\rangle_{0}$ & $\left\langle Q_{8}\right\rangle_{0}$ & $\left\langle Q_{9}\right\rangle_{0}$ & $\left\langle Q_{7}\right\rangle_{2}$ & $\left\langle Q_{8}\right\rangle_{2}$ & $\left\langle Q_{9}\right\rangle_{2}$ & $P_{i}$ & $\frac{\Lambda}{\mathrm{TeV}}$ & \\
\hline$C_{V L L}^{u}$ & 10.7 & -7.4 & -0.0 & 0.2 & -0.07 & -0.04 & 6.32 & 1.6 & 0.8 & -141.8 & $-4.3 \pm 1.0$ & 1.0 & 65 \\
\hline$C_{V L R}^{u}$ & 0.1 & -0.2 & 3.7 & 3.6 & 7.28 & 7.73 & -0.06 & -163.4 & -173.4 & 1.4 & $-125.8 \pm 9.4$ & 9.4 & 354 \\
\hline$\widetilde{C}_{V L L}^{u}$ & -13.6 & 16.4 & 0.2 & -1.2 & -0.01 & & 6.37 & 0.3 & 0.1 & -143.0 & $1.5 \pm 1.7$ & 1.7 & 38 \\
\hline$\widetilde{C}_{V L R}^{u}$ & 0.7 & -1.3 & 0.4 & 13.4 & -0.04 & 30.62 & -0.04 & 1.0 & -687.4 & 0.9 & $-435.5 \pm 33.6$ & 33.6 & 659 \\
\hline$C_{V L L}^{d}$ & 6.6 & -0.6 & 0.1 & -0.9 & 0.04 & 0.03 & -6.26 & -0.9 & -0.6 & 140.6 & $2.3 \pm 0.6$ & 0.6 & 48 \\
\hline$C_{V L R}^{d}$ & 0.1 & -0.3 & 7.5 & 7.5 & -7.41 & -7.84 & 0.03 & 166.4 & 176.1 & -0.7 & $122.9 \pm 10.0$ & 10.0 & 350 \\
\hline$C_{S L R}^{d}$ & -0.3 & 0.7 & -0.2 & -14.5 & -0.01 & 15.51 & -0.01 & 0.2 & -348.2 & 0.2 & $-214.4 \pm 19.0$ & 19.0 & 462 \\
\hline$C_{V L L}^{S}$ & 0.3 & -0.6 & 0.1 & -0.9 & 0.04 & 0.03 & 0.03 & -0.9 & -0.6 & -0.8 & $-0.4 \pm 0.1$ & 0.1 & 18 \\
\hline$C_{V L R}^{S}$ & 0.1 & -0.3 & 0.1 & -0.3 & 0.03 & 0.02 & 0.03 & -0.8 & -0.4 & -0.7 & $-0.3 \pm 0.0$ & 0.0 & 17 \\
\hline$C_{S L R}^{S}$ & 0.3 & -0.7 & 0.2 & -1.0 & 0.01 & 0.01 & 0.01 & -0.2 & -0.2 & -0.2 & $-0.0 \pm 0.1$ & 0.1 & 6 \\
\hline$C_{V L L}^{c}$ & -0.1 & 0.2 & -0.0 & 0.2 & -0.07 & -0.04 & -0.06 & 1.6 & 0.8 & 1.4 & $0.7 \pm 0.1$ & 0.1 & 25 \\
\hline$C_{V L R}^{c}$ & 0.1 & -0.2 & 0.1 & -0.3 & -0.07 & -0.03 & -0.06 & 1.6 & 0.8 & 1.4 & $0.7 \pm 0.1$ & 0.1 & 26 \\
\hline$\tilde{C}_{V L L}^{c}$ & 0.4 & -0.7 & 0.2 & -1.2 & -0.01 & & -0.01 & 0.3 & 0.1 & 0.2 & $0.2 \pm 0.2$ & 0.2 & 13 \\
\hline$\widetilde{C}_{V L R}^{c}$ & 0.7 & -1.3 & 0.4 & -1.9 & -0.04 & -0.01 & -0.04 & 1.0 & 0.2 & 0.9 & $0.4 \pm 0.2$ & 0.2 & 20 \\
\hline$C_{V L L}^{b}$ & -0.0 & 0.0 & -0.0 & 0.1 & 0.02 & 0.02 & 0.02 & -0.6 & -0.4 & -0.5 & $-0.3 \pm 0.0$ & 0.0 & 17 \\
\hline$C_{V L R}^{b}$ & 0.0 & -0.1 & 0.0 & -0.1 & 0.02 & 0.02 & 0.02 & -0.6 & -0.4 & -0.5 & $-0.3 \pm 0.0$ & 0.0 & 16 \\
\hline$\widetilde{C}_{V L L}^{b}$ & 0.3 & -0.4 & 0.1 & -0.8 & & 0.01 & & -0.1 & -0.2 & -0.1 & $-0.0 \pm 0.1$ & 0.1 & 4 \\
\hline$\widetilde{C}_{V L R}^{b}$ & 0.4 & -0.6 & 0.1 & -1.1 & 0.01 & 0.01 & 0.01 & -0.2 & -0.3 & -0.2 & $-0.1 \pm 0.1$ & 0.1 & 8 \\
\hline
\end{tabular}

Table 7 Coefficients $p_{i j}^{(I)}$ and $P_{i}$ of the master formula (18)-(19) as well as suppression scale $\Lambda$ (last column) from Wilson coefficients of Class $\mathrm{B}$ only entering via RG mixing into the chromo-magnetic dipole operator

\begin{tabular}{|c|c|c|c|c|}
\hline & $\left\langle O_{8 g}\right\rangle_{0}$ & $P_{i}$ & $\frac{\Lambda}{\mathrm{TeV}}$ & \\
\hline$C_{8 g}$ & -105.5 & $-0.4 \pm 0.1$ & 0.1 & 18 \\
\hline$C_{S L L}^{s}$ & -15.5 & $-0.0 \pm 0.0$ & 0.0 & 7 \\
\hline$C_{T L L}^{s}$ & 44.4 & $0.2 \pm 0.0$ & 0.0 & 12 \\
\hline$C_{S L L}^{c}$ & 72.9 & $0.2 \pm 0.1$ & 0.1 & 15 \\
\hline$C_{T L L}^{c}$ & 42.6 & $0.1 \pm 0.0$ & 0.0 & 11 \\
\hline$\widetilde{C}_{S L L}^{c}$ & 62.5 & $0.2 \pm 0.1$ & 0.1 & 14 \\
\hline$\widetilde{C}_{T L L}^{c}$ & 1621.3 & $5.4 \pm 1.8$ & 1.8 & 73 \\
\hline$C_{S L L}^{b}$ & 106.2 & $0.4 \pm 0.1$ & 0.1 & 18 \\
\hline$C_{T L L}^{b}$ & 32.4 & $0.1 \pm 0.0$ & 0.0 & 10 \\
\hline$\widetilde{C}_{S L L}^{b}$ & 103.7 & $0.3 \pm 0.1$ & 0.1 & 18 \\
\hline$\widetilde{C}_{T L L}^{b}$ & 4093.5 & $13.5 \pm 4.3$ & 4.3 & 116 \\
\hline
\end{tabular}

Table 8 Coefficients $p_{i j}^{(I)}$ and $P_{i}$ of the master formula (18)-(19) as well as suppression scale $\Lambda$ (last column) from RLRL type operators of Class $\mathrm{C}$ with flavour structure $(\bar{s} d)(\bar{u} u)$

\begin{tabular}{|c|c|c|c|c|c|c|c|c|c|c|}
\hline & $\left\langle O_{8 g}\right\rangle_{0}$ & $\left\langle Q_{1}^{\mathrm{SLL}, u}\right\rangle_{0}$ & $\left\langle Q_{2}^{\mathrm{SLL}, u}\right\rangle_{0}$ & $\left\langle Q_{3}^{\mathrm{SLL}, u}\right\rangle_{0}$ & $\left\langle Q_{4}^{\mathrm{SLL}, u}\right\rangle_{0}$ & $\left\langle Q_{1}^{\mathrm{SLL}, d}\right\rangle_{2}$ & $\left\langle Q_{2}^{\mathrm{SLL}, d}\right\rangle_{2}$ & $P_{i}$ & $\frac{\Lambda}{\mathrm{TeV}}$ & \\
\hline$C_{S L L}^{u}$ & 0.2 & -14.3 & -206.4 & 13.9 & -4.5 & 119.0 & -2541.5 & $-74.1 \pm 15.9$ & 15.9 & 272 \\
\hline$C_{T L L}^{u}$ & 0.1 & -163.3 & 50.3 & 7.9 & 36.6 & 3625.2 & 5846.8 & $161.8 \pm 36.3$ & 36.3 & 402 \\
\hline$\widetilde{C}_{S L L}^{u}$ & 0.1 & -62.8 & -62.0 & 10.8 & -0.5 & -534.9 & 496.7 & $15.6 \pm 3.3$ & 3.3 & 124 \\
\hline$\widetilde{C}_{T L L}^{u}$ & 3.5 & -350.0 & -475.8 & 176.7 & -38.6 & 1075.2 & 17591.7 & $508.6 \pm 107.6$ & 107.6 & 713 \\
\hline
\end{tabular}


Table 9 Coefficients $p_{i j}^{(I)}$ and $P_{i}$ of the master formula (18)-(19) as well as suppression scale $\Lambda$ (last column) from RLRL type operators of Class D with flavour structure $(\bar{s} d)(\bar{d} d)$

\begin{tabular}{lccccccc}
\hline & $\left\langle O_{8 g}\right\rangle_{0}$ & $\left\langle Q_{1}^{\mathrm{SLL}, d}\right\rangle_{0}$ & $\left\langle Q_{1}^{\mathrm{SLL}, d}\right\rangle_{2}$ & $\left\langle Q_{2}^{\mathrm{SLL}, d}\right\rangle_{0}$ & $\left\langle Q_{2}^{\mathrm{SLL}, d}\right\rangle_{2}$ & $P_{i}$ & \multicolumn{1}{c}{} \\
\hline$C_{S L L}^{d}$ & -0.8 & 6.1 & -137.4 & -111.1 & 2493.8 & $87.4 \pm 15.9$ & 15.9 \\
$C_{T L L}^{d}$ & 2.2 & 162.6 & -3649.5 & 254.3 & -5708.9 & $-190.9 \pm 35.4$ & 35.4 \\
\hline
\end{tabular}

Table 10 Coefficients $p_{i j}^{(I)}$ and $P_{i}$ of the master formula (18)-(19) as well as suppression scale $\Lambda$ (last column) from RLLR type operators of Class E with flavour structure $(\bar{s} d)(\bar{u} u)$. Empty entries correspond to vanishing coefficients

\begin{tabular}{lcccccc}
\hline & $\left\langle Q_{7}\right\rangle_{2}$ & $\left\langle Q_{8}\right\rangle_{2}$ & $\left\langle Q_{1}^{\mathrm{SLR}, u}\right\rangle_{0}$ & $\left\langle Q_{2}^{\mathrm{SLR}, u}\right\rangle_{0}$ & $P_{i}$ & $\Lambda$ \\
\hline$C_{S L R}^{u}$ & & & -187.4 & $266.2 \pm 20.7$ & 20.7 \\
$\widetilde{C}_{S L R}^{u}$ & -840.6 & 88.8 & -45 & -47.5 & $59.7 \pm 5.0$ & 515 \\
\hline
\end{tabular}

Table 11 List of the dimension-six four-fermion $\left(\psi^{4}\right)$ operators in SMEFT that contribute to $s \rightarrow d$ transitions at tree level or via mixing. Flavour indices on the quark and lepton fields are $i j k l$

\begin{tabular}{llll}
\hline$(\bar{L} R)(\bar{R} L)$ or $(\bar{L} R)(\bar{L} R)$ & $(\bar{L} L)(\bar{L} L)$ \\
\hline $\mathcal{O}_{\ell e d q}$ & $\left(\bar{\ell}_{i}^{a} e_{j}\right)\left(\bar{d}_{k} q_{l}^{a}\right)$ & $\mathcal{O}_{q q}^{(1)}$ & $\left(\bar{q}_{i} \gamma_{\mu} q_{j}\right)\left(\bar{q}_{k} \gamma^{\mu} q_{l}\right)$ \\
$\mathcal{O}_{q u q d}^{(1)}$ & $\left(\bar{q}_{i}^{a} u_{j}\right) \varepsilon_{a b}\left(\bar{q}_{k}^{b} d_{l}\right)$ & $\mathcal{O}_{\ell q}^{(1)}$ & $\left(\bar{\ell}_{i} \gamma_{\mu} \ell_{j}\right)\left(\bar{q}_{k} \gamma^{\mu} q_{l}\right)$ \\
$\mathcal{O}_{q u q d}^{(8)}$ & $\left(\bar{q}_{i}^{a} T^{A} u_{j}\right) \varepsilon_{a b}\left(\bar{q}_{k}^{b} T^{A} d_{l}\right)$ & $\mathcal{O}_{q q}^{(3)}$ & $\left(\bar{q}_{i} \gamma_{\mu} \tau^{I} q_{j}\right)\left(\bar{q}_{k} \gamma^{\mu} \tau^{I} q_{l}\right)$ \\
$(\bar{L} L)(\bar{R} R)$ & $\mathcal{O}_{\ell q}^{(3)}$ & $\left(\bar{\ell}_{i} \gamma_{\mu} \tau^{I} \ell_{j}\right)\left(\bar{q}_{k} \gamma^{\mu} \tau^{I} q_{l}\right)$ \\
$\mathcal{O}_{\ell u}$ & $\left(\bar{\ell}_{i} \gamma_{\mu} \ell_{j}\right)\left(\bar{u}_{k} \gamma^{\mu} u_{l}\right)$ & & \\
$\mathcal{O}_{\ell d}$ & $\left(\bar{\ell}_{i} \gamma_{\mu} \ell_{j}\right)\left(\bar{d}_{k} \gamma^{\mu} d_{l}\right)$ & $(\bar{R} R)(\bar{R} R)$ \\
$\mathcal{O}_{q e}$ & $\left(\bar{q}_{i} \gamma_{\mu} q_{j}\right)\left(\bar{e}_{k} \gamma^{\mu} e_{l}\right)$ & $\mathcal{O}_{d d}$ & $\left(\bar{d}_{i} \gamma_{\mu} d_{j}\right)\left(\bar{d}_{k} \gamma^{\mu} d_{l}\right)$ \\
$\mathcal{O}_{q u}^{(1)}$ & $\left(\bar{q}_{i} \gamma_{\mu} q_{j}\right)\left(\bar{u}_{k} \gamma^{\mu} u_{l}\right)$ & $\mathcal{O}_{e u}$ & $\left(\bar{e}_{i} \gamma_{\mu} e_{j}\right)\left(\bar{u}_{k} \gamma^{\mu} u_{l}\right)$ \\
$\mathcal{O}_{q d}^{(1)}$ & $\left(\bar{q}_{i} \gamma_{\mu} q_{j}\right)\left(\bar{d}_{k} \gamma^{\mu} d_{l}\right)$ & $\mathcal{O}_{e d}$ & $\left(\bar{e}_{i} \gamma_{\mu} e_{j}\right)\left(\bar{d}_{k} \gamma^{\mu} d_{l}\right)$ \\
$\mathcal{O}_{q u}^{(8)}$ & $\left(\bar{q}_{i} \gamma_{\mu} T^{A} q_{j}\right)\left(\bar{u}_{k} \gamma^{\mu} T^{A} u_{l}\right)$ & $\mathcal{O}_{u d}^{(1)}$ & $\left(\bar{u}_{i} \gamma_{\mu} u_{j}\right)\left(\bar{d}_{k} \gamma^{\mu} d_{l}\right)$ \\
$\mathcal{O}_{q d}^{(8)}$ & $\left(\bar{q}_{i} \gamma_{\mu} T^{A} q_{j}\right)\left(\bar{d}_{k} \gamma^{\mu} T^{A} d_{l}\right)$ & $\mathcal{O}_{u d}^{(8)}$ & $\left(\bar{u}_{i} \gamma_{\mu} T^{A} u_{j}\right)\left(\bar{d}_{k} \gamma^{\mu} T^{A} d_{l}\right)$ \\
\hline & & &
\end{tabular}

- The $\psi^{4}$ semi-leptonic operators $\mathcal{O}_{\ell q}^{(1,3)}, \mathcal{O}_{q e}, \mathcal{O}_{\ell d}$, $\mathcal{O}_{e d}, \mathcal{O}_{\ell e d q}$.

We follow the SMEFT conventions of Ref. [45] and provide the definitions of the above operators in tables 11 and 12, as well as those operators that mix into Classes A-C operators listed in tables 13 and 14.

\section{E. SMEFT matching conditions}

\section{E.1 Four-quark operators}

The tree-level matching of the SMEFT four-quark operators yields the following non-vanishing matching conditions for the Wilson coefficients in the $\Delta S=1$ EFT (61),
Table 12 Dimension-six electro- and chromo-magnetic dipole $\left(\psi^{2} H X\right)$ and $\psi^{2} H^{2} D$ operators in SMEFT

\begin{tabular}{llll}
\hline$\psi^{2} X H$ & $\psi^{2} H^{2} D$ \\
\hline $\mathcal{O}_{u B}$ & $\left(\bar{q}_{i} \sigma^{\mu \nu} u_{j}\right) \tilde{H} B_{\mu \nu}$ & $\mathcal{O}_{H q}^{(1)}$ & $\left(H^{\dagger} i \overleftrightarrow{D}_{\mu} H\right)\left(\bar{q}_{i} \gamma^{\mu} q_{j}\right)$ \\
$\mathcal{O}_{d B}$ & $\left(\bar{q}_{i} \sigma^{\mu \nu} d_{j}\right) H B_{\mu \nu}$ & $\mathcal{O}_{H q}^{(3)}$ & $\left(H^{\dagger} i \overleftrightarrow{D}_{\mu}^{I} H\right)\left(\bar{q}_{i} \tau^{I} \gamma^{\mu} q_{j}\right)$ \\
$\mathcal{O}_{u W}$ & $\left(\bar{q}_{i} \sigma^{\mu \nu} u_{j}\right) \tau^{I} \tilde{H} W_{\mu \nu}^{I}$ & $\mathcal{O}_{H u}$ & $\left(H^{\dagger} i \overleftrightarrow{D}_{\mu} H\right)\left(\bar{u}_{i} \gamma^{\mu} u_{j}\right)$ \\
$\mathcal{O}_{d W}$ & $\left(\bar{q}_{i} \sigma^{\mu \nu} d_{j}\right) \tau^{I} H W_{\mu \nu}^{I}$ & $\mathcal{O}_{H d}$ & $\left(H^{\dagger} i \overleftrightarrow{D}_{\mu} H\right)\left(\bar{d}_{i} \gamma^{\mu} d_{j}\right)$ \\
$\mathcal{O}_{u G}$ & $\left(\bar{q}_{i} \sigma^{\mu \nu} T^{A} u_{j}\right) \tilde{H} G_{\mu \nu}^{A}$ & $\mathcal{O}_{H u d}$ & $\left(\widetilde{H}^{\dagger} i D_{\mu} H\right)\left(\bar{u}_{i} \gamma^{\mu} d_{j}\right)$ \\
$\mathcal{O}_{d G}$ & $\left(\bar{q}_{i} \sigma^{\mu \nu} T^{A} d_{j}\right) H G_{\mu \nu}^{A}$ & & \\
\hline
\end{tabular}

$C_{V L L}^{d_{i}}=\left[\mathcal{C}_{q q}^{(1)}\right]_{21 i i}+\left[\mathcal{C}_{q q}^{(3)}\right]_{21 i i}$,

$\widetilde{C}_{V L L}^{d_{i}}=\left[\mathcal{C}_{q q}^{(1)}\right]_{2 i i 1}+\left[\mathcal{C}_{q q}^{(3)}\right]_{2 i i 1}$,

$C_{V L L}^{u_{i}}=\sum_{j k} V_{i j} V_{i k}^{*}\left(\left[\mathcal{C}_{q q}^{(1)}\right]_{21 j k}+\left[\mathcal{C}_{q q}^{(1)}\right]_{j k 21}\right.$

$$
\left.-\left[\mathcal{C}_{q q}^{(3)}\right]_{21 j k}-\left[\mathcal{C}_{q q}^{(3)}\right]_{j k 21}\right)
$$

$\widetilde{C}_{V L L}^{u_{i}}=2 \sum_{j k} V_{i j} V_{i k}^{*}\left(\left[\mathcal{C}_{q q}^{(3)}\right]_{j 12 k}+\left[\mathcal{C}_{q q}^{(3)}\right]_{2 k j 1}\right)$,

$C_{V R R}^{d_{i}}=\left[\mathcal{C}_{d d}\right]_{21 i i}, \widetilde{C}_{V R R}^{d_{i}}=\left[\mathcal{C}_{d d}\right]_{2 i i 1}$,

$C_{V R R}^{u_{i}}=\left[\mathcal{C}_{u d}^{(1)}\right]_{i i 21}-\frac{1}{6}\left[\mathcal{C}_{u d}^{(8)}\right]_{i i 21}, \widetilde{C}_{V R R}^{u_{i}}=\frac{1}{2}\left[\mathcal{C}_{u d}^{(8)}\right]_{i i 21}$,

$C_{V L R}^{u_{i}}=\left[\mathcal{C}_{q u}^{(1)}\right]_{21 i i}-\frac{1}{6}\left[\mathcal{C}_{q u}^{(8)}\right]_{21 i i}, \widetilde{C}_{V L R}^{u_{i}}=\frac{1}{2}\left[\mathcal{C}_{q u}^{(8)}\right]_{21 i i}$

$C_{V R L}^{u_{i}}=\sum_{j k} V_{i j} V_{i k}^{*}\left(\left[\mathcal{C}_{q d}^{(1)}\right]_{j k 21}-\frac{1}{6}\left[\mathcal{C}_{q d}^{(8)}\right]_{j k 21}\right)$,

$\widetilde{C}_{V R L}^{u_{i}}=\frac{1}{2} \sum_{j k} V_{i j} V_{i k}^{*}\left[\mathcal{C}_{q d}^{(8)}\right]_{j k 21}$, 
Table 13 List of four-quark operators in Classes A-C that receive at one-loop in SMEFT contributions from non-leptonic $\psi^{4}$ and $\psi^{2} H^{2} D$ operators through mixing via the top-quark Yukawa coupling. Selfmixing is included. We have omitted $\mathcal{O}_{H u d}$ (Class E) that only mixes with itself through the top Yukawa coupling

\begin{tabular}{lll}
\hline Class A & non-leptonic $\psi^{4}$ & $\psi^{2} H^{2} D$ \\
\hline $\mathcal{O}_{q q}^{(1)}$ & $\mathcal{O}_{q q}^{(1)}, \mathcal{O}_{q u}^{(1,8)}$ & $\mathcal{O}_{H q}^{(1)}$ \\
$\mathcal{O}_{q q}^{(3)}$ & $\mathcal{O}_{q q}^{(3)}, \mathcal{O}_{q u}^{(8)}$ & $\mathcal{O}_{H q}^{(3)}$ \\
$\mathcal{O}_{q u}^{(1)}$ & $\mathcal{O}_{q u}^{(1,8)}, \mathcal{O}_{q q}^{(1,3)}, \mathcal{O}_{u u}$ & $\mathcal{O}_{H q}^{(1)}, \mathcal{O}_{H u}$ \\
$\mathcal{O}_{q u}^{(8)}$ & $\mathcal{O}_{q u}^{(1,8)}, \mathcal{O}_{q q}^{(1,3)}, \mathcal{O}_{u u}$ & - \\
$\mathcal{O}_{q d}^{(1)}$ & $\mathcal{O}_{u d}^{(1)}, \mathcal{O}_{q d}^{(1)}$ & $\mathcal{O}_{H d}$ \\
$\mathcal{O}_{q d}^{(8)}$ & $\mathcal{O}_{u d}^{(8)}, \mathcal{O}_{q d}^{(8)}$ & - \\
$\mathcal{O}_{u d}^{(1)}$ & $\mathcal{O}_{u d}^{(1)}, \mathcal{O}_{q d}^{(1)}$ & $\mathcal{O}_{H d}$ \\
$\mathcal{O}_{u d}^{(8)}$ & $\mathcal{O}_{u d}^{(8)}, \mathcal{O}_{q d}^{(8)}$ & - \\
$\mathcal{O}_{d d}$ & - & - \\
$\mathcal{O}_{H q}^{(1)}$ & $\mathcal{O}_{q q}^{(1,3)}, \mathcal{O}_{q u}^{(1)}$ & $\mathcal{O}_{H q}^{(1)}$ \\
$\mathcal{O}_{H q}^{(3)}$ & $\mathcal{O}_{q q}^{(1,3)}$ & $\mathcal{O}_{H q}^{(3)}$ \\
$\mathcal{O}_{H d}$ & $\mathcal{O}_{q d}^{(1)}, \mathcal{O}_{u d}^{(1)}$ & $\mathcal{O}_{H d}$ \\
\hline Class B + C & non-leptonic $\psi^{4}$ & $\psi^{2} H X$ \\
\hline $\mathcal{O}_{d G}$ & $\mathcal{O}_{q u q d}^{(1,8)}$ & $\mathcal{O}_{d G}$ \\
$\mathcal{O}_{q u q d}^{(1)}$ & $\mathcal{O}_{q u q d}^{(1,8)}$ & $\mathcal{O}_{q u q d}^{(8)}$ \\
$\mathcal{O}_{q u q d}^{(8)}$ & & \\
\hline
\end{tabular}

$C_{V L R}^{d_{i}}=\left[\mathcal{C}_{q d}^{(1)}\right]_{21 i i}-\frac{1}{6}\left[\mathcal{C}_{q d}^{(8)}\right]_{21 i i}, \widetilde{C}_{V L R}^{d_{i}}=\frac{1}{2}\left[\mathcal{C}_{q d}^{(8)}\right]_{21 i i}$,

$C_{V R L}^{d_{i}}=\left[\mathcal{C}_{q d}^{(1)}\right]_{i i 21}-\frac{1}{6}\left[\mathcal{C}_{q d}^{(8)}\right]_{i i 21}, \widetilde{C}_{V R L}^{d_{i}}=\frac{1}{2}\left[\mathcal{C}_{q d}^{(8)}\right]_{i i 21}$,

$C_{S R L}^{d_{i}}=-\left[\mathcal{C}_{q d}^{(8)}\right]_{2 i i 1}, \widetilde{C}_{S R L}^{d_{i}}=-2\left[\mathcal{C}_{q d}^{(1)}\right]_{2 i i 1}+\frac{1}{3}\left[\mathcal{C}_{q d}^{(8)}\right]_{2 i i 1}$,

$$
C_{S L R}^{d_{i}}=-\left[\mathcal{C}_{q d}^{(8)}\right]_{i 12 i}, \widetilde{C}_{S L R}^{d_{i}}=-2\left[\mathcal{C}_{q d}^{(1)}\right]_{i 12 i}+\frac{1}{3}\left[\mathcal{C}_{q d}^{(8)}\right]_{i 12 i}
$$

$$
\begin{aligned}
C_{S R R}^{u_{i}}= & \sum_{j} V_{i j}\left(\left[\mathcal{C}_{q u q d}^{(1)}\right]_{j i 21}+\frac{1}{4}\left[\mathcal{C}_{q u q d}^{(8)}\right]_{2 i j 1}\right. \\
& \left.-\frac{1}{6}\left[\mathcal{C}_{q u q d}^{(8)}\right]_{j i 21}\right)
\end{aligned}
$$

$$
\begin{aligned}
\widetilde{C}_{S R R}^{u_{i}}= & \sum_{j} V_{i j}\left(\frac{1}{2}\left[\mathcal{C}_{q u q d}^{(1)}\right]_{2 i j 1}-\frac{1}{12}\left[\mathcal{C}_{q u q d}^{(8)}\right]_{2 i j 1}\right. \\
& \left.+\frac{1}{2}\left[\mathcal{C}_{q u q d}^{(8)}\right]_{j i 21}\right)
\end{aligned}
$$

$$
\begin{aligned}
C_{S L L}^{u_{i}}= & \sum_{j} V_{i j}^{*}\left(\left[\mathcal{C}_{q u q d}^{(1)}\right]_{j i 12}^{*}+\frac{1}{4}\left[\mathcal{C}_{q u q d}^{(8)}\right]_{1 i j 2}^{*}\right. \\
& \left.-\frac{1}{6}\left[\mathcal{C}_{q u q d}^{(8)}\right]_{j i 12}^{*}\right), \\
\widetilde{C}_{S L L}^{u_{i}}= & \sum_{j} V_{i j}^{*}\left(\frac{1}{2}\left[\mathcal{C}_{q u q d}^{(1)}\right]_{1 i j 2}^{*}-\frac{1}{12}\left[\mathcal{C}_{q u q d}^{(8)}\right]_{1 i j 2}^{*}\right. \\
& \left.+\frac{1}{2}\left[\mathcal{C}_{q u q d}^{(8)}\right]_{j i 12}^{*}\right), \\
C_{T R R}^{u_{i}}= & \frac{1}{16} \sum_{j} V_{i j}\left[\mathcal{C}_{q u q d}^{(8)}\right]_{2 i j 1}, \\
\widetilde{C}_{T R R}^{u_{i}}= & \sum_{j} V_{i j}\left(\frac{1}{8}\left[\mathcal{C}_{q u q d}^{(1)}\right]_{2 i j 1}-\frac{1}{48}\left[\mathcal{C}_{q u q d}^{(8)}\right]_{2 i j 1}\right), \\
C_{T L L}^{u_{i}}= & \frac{1}{16} \sum_{j} V_{i j}^{*}\left[\mathcal{C}_{q u q d}^{(8)}\right]_{1 i j 2}^{*}, \\
\widetilde{C}_{T L L}^{u_{i}}= & \sum_{j} V_{i j}^{*}\left(\frac{1}{8}\left[\mathcal{C}_{q u q d}^{(1)}\right]_{1 i j 2}^{*}-\frac{1}{48}\left[\mathcal{C}_{q u q d}^{(8)}\right]_{1 i j 2}^{*}\right) .
\end{aligned}
$$

where $V$ is the CKM matrix and we have explicitly written the sum over $j$ on the right-hand side where necessary, while $i$ is not to be summed over.

\section{E.2 Modified $Z^{0}$ and $W^{ \pm}$couplings}

In addition to the direct matching of four-quark SMEFT operators onto four-quark $\Delta S=1 \mathrm{EFT}$ operators, the latter also receive dimension-six matching contributions from diagrams with tree-level $Z^{0}$ or $W^{ \pm}$exchange, with one SM coupling and the other from a SMEFT $\psi^{2} H^{2} D$ operator of modified $Z^{0}$ or $W^{ \pm}$coupling.

The $Z^{0}$ exchanges lead to the following additional matching contributions to vector operators,

$$
\begin{array}{ll}
C_{V L A}^{u_{i}}=2 \zeta_{u_{A}}\left[\mathcal{C}_{H q}^{(1)}+\mathcal{C}_{H q}^{(3)}\right]_{12}^{*}, & C_{V R A}^{u_{i}}=2 \zeta_{u_{A}}\left[\mathcal{C}_{H d}\right]_{12}^{*}, \\
C_{V L A}^{d_{i}}=2 \zeta_{d_{A}}\left[\mathcal{C}_{H q}^{(1)}+\mathcal{C}_{H q}^{(3)}\right]_{12}^{*}, & C_{V R A}^{d_{i}}=2 \zeta_{d_{A}}\left[\mathcal{C}_{H d}\right]_{12}^{*},
\end{array}
$$

where we have written the SM $Z^{0}$ coupling to quarks $q=$ $u, d$ and $A=L, R$, as

$\mathcal{L}_{\mathrm{SM}} \supset \frac{g}{\cos \theta_{W}} \zeta_{q_{A}}\left(\bar{q} \gamma^{\mu} P_{A} q\right) Z_{\mu}^{0}$, 
Table 14 List of SMEFT operators that mix in SMEFT at one-loop with the ones in Classes A-C through gauge couplings. Self-mixing is included. We have omitted $\mathcal{O}_{H u d}$ (Class E) that only mixes with itself through gauge couplings

\begin{tabular}{llll}
\hline Class A & non-leptonic $\psi^{4}$ & semi-leptonic $\psi^{4}$ & $\psi^{2} H^{2} D$ \\
\hline $\mathcal{O}_{q q}^{(1)}$ & $\mathcal{O}_{q q}^{(1,3)}, \mathcal{O}_{q u}^{(1,8)}, \mathcal{O}_{q d}^{(1,8)}$ & $\mathcal{O}_{\ell q}^{(1)}, \mathcal{O}_{q e}$ & $\mathcal{O}_{H q}^{(1)}$ \\
$\mathcal{O}_{q q}^{(3)}$ & $\mathcal{O}_{q q}^{(1,3)}, \mathcal{O}_{q u}^{(8)}, \mathcal{O}_{q d}^{(8)}$ & $\mathcal{O}_{\ell q}^{(3)}$ & $\mathcal{O}_{H q}^{(3)}$ \\
$\mathcal{O}_{q u}^{(1)}$ & $\mathcal{O}_{q q}^{(1,3)}, \mathcal{O}_{q u}^{(1,8)}, \mathcal{O}_{q d}^{(1)}, \mathcal{O}_{u d}^{(1)}, \mathcal{O}_{u u}$ & $\mathcal{O}_{\ell q}^{(1)}, \mathcal{O}_{q e}, \mathcal{O}_{\ell u}, \mathcal{O}_{e u}$ & $\mathcal{O}_{H q}^{(1)}, \mathcal{O}_{H u}$ \\
$\mathcal{O}_{q u}^{(8)}$ & $\mathcal{O}_{q q}^{(1,3)}, \mathcal{O}_{q u}^{(1,8)}, \mathcal{O}_{q d}^{(8)}, \mathcal{O}_{u d}^{(8)}, \mathcal{O}_{u u}$ & & $\mathcal{O}_{H q}^{(1)}, \mathcal{O}_{H d}$ \\
$\mathcal{O}_{q d}^{(1)}$ & $\mathcal{O}_{q q}^{(1,3)}, \mathcal{O}_{q u}^{(1)}, \mathcal{O}_{q d}^{(1), 8)}, \mathcal{O}_{u d}^{(1)}, \mathcal{O}_{d d}$ & $\mathcal{O}_{\ell q}^{(1)}, \mathcal{O}_{q e}, \mathcal{O}_{\ell d}, \mathcal{O}_{e d}$ & \\
$\mathcal{O}_{q d}^{(8)}$ & $\mathcal{O}_{q q}^{(1,3)}, \mathcal{O}_{q u}^{(8)}, \mathcal{O}_{q d}^{(1,8)}, \mathcal{O}_{u d}^{(8)}, \mathcal{O}_{d d}$ & & $\mathcal{O}_{H u}, \mathcal{O}_{H d}$ \\
$\mathcal{O}_{u d}^{(1)}$ & $\mathcal{O}_{u d}^{(1,8)}, \mathcal{O}_{q u}^{(1)}, \mathcal{O}_{q d}^{(1)}, \mathcal{O}_{u u}, \mathcal{O}_{d d}$ & $\mathcal{O}_{\ell u}, \mathcal{O}_{\ell d}, \mathcal{O}_{e u}, \mathcal{O}_{e d}$ & $\mathcal{O}_{H d}$ \\
$\mathcal{O}_{u d}^{(8)}$ & $\mathcal{O}_{q u}^{(8)}, \mathcal{O}_{q d}^{(8)}, \mathcal{O}_{u d}^{(1,8)}, \mathcal{O}_{u u}, \mathcal{O}_{d d}$ & & $\mathcal{O}_{H q}^{(1)}$ \\
$\mathcal{O}_{d d}$ & $\mathcal{O}_{q d}^{(1,8)}, \mathcal{O}_{u d}^{(1,8)}, \mathcal{O}_{d d}$ & $\mathcal{O}_{\ell d}, \mathcal{O}_{e d}$ & $\mathcal{O}_{H q}^{(3)}$ \\
$\mathcal{O}_{H q}^{(1)}$ & $\mathcal{O}_{q q}^{(1), 3)}, \mathcal{O}_{q u}^{(1)}, \mathcal{O}_{q d}^{(1)}$ & $\mathcal{O}_{\ell q}^{(1)}, \mathcal{O}_{q e}$ & $\mathcal{O}_{H d}$ \\
$\mathcal{O}_{H q}^{(3)}$ & $\mathcal{O}_{q q}^{(1,3)}$ & $\mathcal{O}_{\ell q}^{(3)}$ & \\
$\mathcal{O}_{H d}$ & $\mathcal{O}_{q d}^{(1)}, \mathcal{O}_{u d}^{(1)}, \mathcal{O}_{d d}, \mathcal{O}_{u d}^{(1)}$ & $\mathcal{O}_{\ell d}, \mathcal{O}_{e d}$ & \\
\hline Class B+C & non-leptonic $\psi^{4}$ & $\psi^{2} H X$ & \\
\hline $\mathcal{O}_{d G}$ & $\mathcal{O}_{d G}^{(1,8)}, \mathcal{O}_{d B}, \mathcal{O}_{d W}$ & \\
$\mathcal{O}_{q u q d}^{(1)}$ & $\mathcal{O}_{q u q}^{(1,8)}$ & $\mathcal{O}_{u G}, \mathcal{O}_{u B}, \mathcal{O}_{u W}, \mathcal{O}_{d B}, \mathcal{O}_{d W}$ & \\
$\mathcal{O}_{q u q d}^{(8)}$ & $\mathcal{O}_{u G}, \mathcal{O}_{d G}, \mathcal{O}_{u B}, \mathcal{O}_{u W}, \mathcal{O}_{d B}, \mathcal{O}_{d W}$ & \\
\hline
\end{tabular}

with

$\zeta_{q_{L}}=T_{3}^{q}-Q_{q} \sin ^{2} \theta_{W}, \quad \zeta_{q_{R}}=-Q_{q} \sin ^{2} \theta_{W}$,

and the $S U(2)_{L}$ coupling $g$.

In the case of $W^{ \pm}$exchange, there are two qualitatively different contributions. The first involves a modified $W^{ \pm}$coupling to left-handed quarks induced by the operator $\mathcal{O}_{H q}^{(3)}$ and affects the matching contribution of $\widetilde{C}_{V L L}^{u_{i}}$,

$\widetilde{C}_{V L L}^{u_{i}}=-2 \sum_{j}\left(\left[\mathcal{C}_{H q}^{(3)}\right]_{j 2}^{*} V_{i d} V_{i j}^{*}+\left[\mathcal{C}_{H q}^{(3)}\right]_{1 j}^{*} V_{i j} V_{i s}^{*}\right)$,

where again the sum over $j$ has been made explicit and $i$ is not to be summed over. Here only the terms with Wilson coefficients $\left[\mathcal{C}_{H q}^{(3)}\right]_{k l}$ with $k l=12,13,23$ are relevant for $\varepsilon^{\prime} / \varepsilon,{ }^{18}$ because for $k=l$ the $\left[\mathcal{C}_{H q}^{(3)}\right]_{k l}$ is manifestly realvalued whereas the accompanying CKM factor is also realvalued $(k=1)$ or has a negligible phase $(k=2)$, such that there is no contribution to $\varepsilon^{\prime} / \varepsilon$.

The second contribution originates from the $W^{ \pm}$coupling to right-handed quarks induced by the operator $\mathcal{O}_{H u d}$. In this

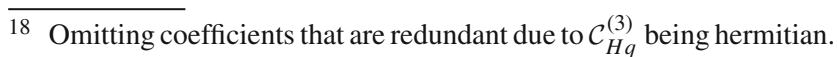

case the only non-vanishing matching conditions are

$\widetilde{C}_{S L R}^{u_{i}}=2 V_{i d}\left[\mathcal{C}_{H u d}\right]_{i 2}^{*}, \quad \widetilde{C}_{S R L}^{u_{i}}=2 V_{i s}^{*}\left[\mathcal{C}_{H u d}\right]_{i 1}$.

Since the operators $\widetilde{C}_{S L R, S R L}^{c}$ do not contribute to $\varepsilon^{\prime} / \varepsilon$ at the one-loop level as discussed in Sect. 2.2, only the case $i=1$ is relevant.

The effect of the right-handed $W^{ \pm}$coupling on $\varepsilon^{\prime} / \varepsilon$ has been discussed recently in $[85,103]$ and of the other $\psi^{2} H^{2} D$ operators in [24-26].

\section{E.3 Dipole operators}

Since we neglect the electro-magnetic dipole operators, the only relevant matching conditions are those of the chromomagnetic operators, that trivially read

$C_{8 g}=\frac{v}{\sqrt{2} m_{s}}\left[\mathcal{C}_{d G}\right]_{12}^{*}, \quad C_{8 g}^{\prime}=\frac{v}{\sqrt{2} m_{s}}\left[\mathcal{C}_{d G}\right]_{21}$,

taking into account our normalization in (6). Here $v \approx$ $246 \mathrm{GeV}$ is the Higgs vacuum expectation value. 


\section{F. RG evolution in SMEFT}

In Table 13 all operators are listed that mix through the large top-quark Yukawa coupling into the four-quark SMEFT operators in Classes A-C. For Class A, these are either fourquark operators or $\psi^{2} H^{2} D$ operators describing modified $W^{ \pm}$and $Z^{0}$ couplings whereas in the case of Classes B-C only scalar four-quark operators contribute. The corresponding mixing through gauge couplings is given in Table 14. For Class A, there are four-quark and semi-leptonic operators and again $\psi^{2} H^{2} D$ operators, whereas in the case of Classes B-C scalar four-quark and dipole operators $\psi^{2} H X$ contribute.

\section{References}

1. NA48 Collaboration, J.R. Batley et al., A Precision measurement of direct $\mathrm{CP}$ violation in the decay of neutral kaons into two pions. Phys. Lett. B 544, 97-112 (2002). arXiv:hep-ex/0208009

2. KTeV Collaboration, A. Alavi-Harati et al., Measurements of direct CP violation, CPT symmetry, and other parameters in the neutral kaon system. Phys. Rev. D 67, 012005 (2003). arXiv:hep-ex/0208007. [Erratum: Phys. Rev.D70,079904(2004)]

3. KTeV Collaboration, E. Abouzaid et al., Precise measurements of Direct CP Violation, CPT symmetry, and other parameters in the neutral Kaon System. Phys. Rev. D 83, 092001 (2011). arXiv: 1011.0127

4. A.J. Buras, M. Jamin, M.E. Lautenbacher, P. H. Weisz, Effective Hamiltonians for $\Delta S=1$ and $\Delta B=1$ nonleptonic decays beyond the leading logarithmic approximation. Nucl. Phys. B 370, 69-104 (1992). [Addendum: Nucl. Phys.B375,501(1992)]

5. A.J. Buras, M. Jamin, M.E. Lautenbacher, P.H. Weisz, Two loop anomalous dimension matrix for $\Delta S=1$ weak nonleptonic decays I: $\mathcal{O}\left(\alpha_{s}^{2}\right)$. Nucl. Phys. B 400, 37-74 (1993). arXiv:hep-ph/9211304

6. A.J. Buras, M. Jamin, M.E. Lautenbacher, Two loop anomalous dimension matrix for Delta $S=1$ weak nonleptonic decays. 2. $\mathrm{O}\left(\alpha \alpha_{s}\right)$. Nucl. Phys. B 400, 75-102 (1993). arXiv:hep-ph/9211321

7. M. Ciuchini, E. Franco, G. Martinelli, L. Reina, $\varepsilon^{\prime} / \varepsilon$ at the Nextto-leading order in QCD and QED. Phys. Lett. B 301, 263-271 (1993). arXiv:hep-ph/9212203

8. A.J. Buras, M. Jamin, M.E. Lautenbacher, The anatomy of $\varepsilon^{\prime} / \varepsilon$ beyond leading logarithms with improved hadronic matrix elements. Nucl. Phys. B 408, 209-285 (1993). arXiv:hep-ph/9303284

9. M. Ciuchini, E. Franco, G. Martinelli, L. Reina, The $\Delta S=$ 1 effective Hamiltonian including next-to-leading order QCD and QED corrections. Nucl. Phys. B 415, 403-462 (1994). arXiv:hep-ph/9304257

10. C. Bobeth, M. Misiak, J. Urban, Photonic penguins at two loops and $m_{t}$-dependence of $B R\left(B \rightarrow X_{s} \ell^{+} \ell^{-}\right)$. Nucl. Phys. B 574, 291-330 (2000). arXiv:hep-ph/9910220

11. A.J. Buras, P. Gambino, U.A. Haisch, Electroweak penguin contributions to nonleptonic $\Delta F=1$ decays at NNLO. Nucl. Phys. B 570, 117-154 (2000). arXiv:hep-ph/9911250

12. M. Gorbahn, U. Haisch, Effective Hamiltonian for non-leptonic $|\Delta F|=1$ decays at NNLO in QCD. Nucl. Phys. B 713, 291-332 (2005). arXiv:hep-ph/0411071

13. J. Brod, M. Gorbahn, $\varepsilon_{K}$ at next-to-next-to-leading order: the charm-top-quark contribution. Phys. Rev. D 82, 094026 (2010). arXiv: 1007.0684
14. M. Cerdà-Sevilla, M. Gorbahn, S. Jäger, A. Kokulu, Towards NNLO accuracy for $\varepsilon^{\prime} / \varepsilon$. J. Phys. Conf. Ser. 800(1), 012008 (2017). arXiv: 1611.08276

15. RBC, UKQCD Collaboration, Z. Bai et al., Standard model prediction for direct $\mathrm{CP}$ violation in $K \rightarrow \pi \pi$ decay. Phys. Rev. Lett. 115(21), 212001 (2015). arXiv: 1505.07863

16. T. Blum et al., $K \rightarrow \pi \pi \Delta I=3 / 2$ decay amplitude in the continuum limit. Phys. Rev. D 91(7), 074502 (2015). arXiv:1502.00263

17. A.J. Buras, M. Gorbahn, S. Jäger, M. Jamin, Improved anatomy of $\varepsilon^{\prime} / \varepsilon$ in the standard model. JHEP 11, 202 (2015). arXiv: 1507.06345

18. T. Kitahara, U. Nierste, P. Tremper, Singularity-free next-toleading order $\Delta S=1$ renormalization group evolution and $\varepsilon / \varepsilon$ in the Standard Model and beyond. JHEP 12, 078 (2016). arXiv: 1607.06727

19. A.J. Buras, J.-M. Gérard, Upper bounds on $\varepsilon^{\prime} / \varepsilon$ parameters $B_{6}^{(1 / 2)}$ and $B_{8}^{(3 / 2)}$ from large N QCD and other news. JHEP 12, 008 (2015). arXiv: 1507.06326

20. A.J. Buras, J.-M. Gérard, Final state interactions in $K \rightarrow \pi \pi$ decays: $\Delta I=1 / 2$ rule vs. $\varepsilon^{\prime} / \varepsilon$. Eur. Phys. J. C 77(1), 10 (2017). arXiv: 1603.05686

21. H. Gisbert, A. Pich, Direct CP violation in $K^{0} \rightarrow \pi \pi$ : standard model status. Rept. Prog. Phys. 81(7), 076201 (2018). arXiv: 1712.06147

22. A.J. Buras, F. De Fazio, $\varepsilon^{\prime} / \varepsilon$ in 331 Models. JHEP 03, 010 (2016). arXiv: 1512.02869

23. A.J. Buras, F. De Fazio, 331 Models facing the tensions in $\Delta F=$ 2 Processes with the Impact on $\varepsilon^{\prime} / \varepsilon, B_{s} \rightarrow \mu^{+} \mu^{-}$and $B \rightarrow$ $K^{*} \mu^{+} \mu^{-}$. JHEP 08, 115 (2016). arXiv:1604.02344

24. C. Bobeth, A.J. Buras, A. Celis, M. Jung, Yukawa enhancement of $Z$-mediated new physics in $\Delta S=2$ and $\Delta B=2$ processes. JHEP 07, 124 (2017). arXiv:1703.04753

25. M. Endo, T. Kitahara, S. Mishima, K. Yamamoto, Revisiting Kaon Physics in General Z Scenario. Phys. Lett. B 771, 37-44 (2017). arXiv: 1612.08839

26. C. Bobeth, A.J. Buras, A. Celis, M. Jung, Patterns of flavour violation in models with vector-like quarks. JHEP 04, 079 (2017). arXiv: 1609.04783

27. M. Blanke, A.J. Buras, S. Recksiegel, Quark flavour observables in the Littlest Higgs model with T-parity after LHC Run 1. Eur. Phys. J. C 76(4), 182 (2016). arXiv: 1507.06316

28. A.J. Buras, D. Buttazzo, R. Knegjens, $K \rightarrow \pi v \bar{v}$ and $\varepsilon^{\prime} / \varepsilon$ in simplified new physics models. JHEP 11, 166 (2015). arXiv: 1507.08672

29. A.J. Buras, New physics patterns in $\varepsilon^{\prime} / \varepsilon$ and $\varepsilon_{K}$ with implications for rare kaon decays and $\Delta M_{K}$. JHEP 04, 071 (2016). arXiv: 1601.00005

30. M. Tanimoto, K. Yamamoto, Probing SUSY with $10 \mathrm{TeV}$ stop mass in rare decays and CP violation of kaon. PTEP 2016(12), 123B02 (2016). arXiv:1603.07960

31. T. Kitahara, U. Nierste, P. Tremper, Supersymmetric explanation of CP violation in $K \rightarrow \pi \pi$ decays. Phys. Rev. Lett. 117(9), 091802 (2016). arXiv: 1604.07400

32. M. Endo, S. Mishima, D. Ueda, K. Yamamoto, Chargino contributions in light of recent $\varepsilon^{\prime} / \varepsilon$. Phys. Lett. B 762, 493-497 (2016). arXiv: 1608.01444

33. A. Crivellin, G. D'Ambrosio, T. Kitahara, U. Nierste, $K \rightarrow \pi \nu \bar{v}$ in the MSSM in light of the $\varepsilon^{\prime} / \varepsilon$ anomaly. Phys. Rev. D 96(1), 015023 (2017). arXiv: 1703.05786

34. M. Endo, T. Goto, T. Kitahara, S. Mishima, D. Ueda, K. Yamamoto, Gluino-mediated electroweak penguin with flavor-violating trilinear couplings. JHEP 04, 019 (2018). arXiv: 1712.04959

35. C.-H. Chen, T. Nomura, $\varepsilon^{\prime} / \varepsilon$ and $K \rightarrow \pi v \bar{v}$ in a two-Higgs doublet model. arXiv:1804.06017 
36. C.-H. Chen, T. Nomura, $\varepsilon^{\prime} / \varepsilon$ from charged-Higgs-induced gluonic dipole operators. arXiv: 1805.07522

37. N. Haba, H. Umeeda, T. Yamada, $\varepsilon^{\prime} / \varepsilon$ Anomaly and Neutron EDM in $S U(2)_{L} \times S U(2)_{R} \times U(1)_{B-L}$ model with Charge Symmetry. JHEP 05, 052 (2018). arXiv: 1802.09903

38. N. Haba, H. Umeeda, T. Yamada, Direct CP Violation in Cabibbofavored charmed meson decays and $\varepsilon^{\prime} / \varepsilon$ in $S U(2)_{L} \times S U(2)_{R} \times$ $U(1)_{B-L}$ model. arXiv: 1806.03424

39. S. Matsuzaki, K. Nishiwaki, K. Yamamoto, Simultaneous interpretation of $K$ and $B$ anomalies in terms of chiral-flavorful vectors. arXiv: 1806.02312

40. C. Bobeth, A.J. Buras, Leptoquarks meet $\varepsilon^{\prime} / \varepsilon$ and rare Kaon processes. JHEP 02, 101 (2018). arXiv:1712.01295

41. ETM Collaboration, M. Constantinou, M. Costa, R. Frezzotti, V. Lubicz, G. Martinelli, D. Meloni, H. Panagopoulos, S. Simula, $K \rightarrow \pi$ matrix elements of the chromomagnetic operator on the lattice. Phys. Rev. D 97(7), 074501 (2018). arXiv:1712.09824

42. A.J. Buras, J.-M. Gérard, $K \rightarrow \pi \pi$ and $K-\pi$ Matrix elements of the chromomagnetic operators from dual QCD. JHEP 07, 126 (2018). arXiv: 1803.08052

43. J. Aebischer, A.J. Buras, J.-M. Gérard, BSM hadronic matrix elements for $\epsilon^{\prime} / \epsilon$ and $K \rightarrow \pi \pi$ Decays in the Dual QCD approach. JHEP 02, 021 (2019). https://doi.org/10.1007/JHEP02(2019)021 arXiv: 1807.01709

44. W. Buchmüller, D. Wyler, Effective Lagrangian analysis of new interactions and flavor conservation. Nucl. Phys. B 268, 621-653 (1986)

45. B. Grzadkowski, M. Iskrzynski, M. Misiak, J. Rosiek, Dimensionsix terms in the standard model lagrangian. JHEP 10, 085 (2010). arXiv: 1008.4884

46. J. Aebischer, C. Bobeth, A.J. Buras, J.-M. Gérard, D.M. Straub, Master formula for $\varepsilon^{\prime} / \varepsilon$ beyond the standard model. arXiv: 1807.02520

47. V. Cirigliano, G. Ecker, H. Neufeld, A. Pich, Isospin breaking in $K \rightarrow \pi \pi$ decays. Eur. Phys. J. C 33, 369-396 (2004). arXiv:hep-ph/0310351

48. V. Cirigliano, A. Pich, G. Ecker, H. Neufeld, Isospin violation in $\varepsilon^{\prime}$. Phys. Rev. Lett. 91, 162001 (2003). arXiv:hep-ph/0307030

49. G. Buchalla, A.J. Buras, M.E. Lautenbacher, Weak decays beyond leading logarithms. Rev. Mod. Phys. 68, 1125-1144 (1996). arXiv:hep-ph/9512380

50. W.A. Bardeen, A.J. Buras, J.M. Gérard, A consistent analysis of the $\Delta I=1 / 2$ rule for $\mathrm{K}$ decays. Phys. Lett. B 192, 138-144 (1987)

51. A.J. Buras, J.M. Gérard, $1 / N$ expansion for Kaons. Nucl. Phys. B 264, 371-392 (1986)

52. A.J. Buras, J.-M. Gérard, W.A. Bardeen, Large $N$ approach to Kaon decays and mixing 28 years later: $\Delta I=1 / 2$ rule, $\hat{B}_{K}$ and $\Delta M_{K}$. Eur. Phys. J. C 74, 2871 (2014). arXiv:1401.1385

53. S. Bertolini, J.O. Eeg, M. Fabbrichesi, Studying $\varepsilon^{\prime} / \varepsilon$ in the chiral quark model: $\gamma_{5}$ scheme independence and NLO hadronic matrix elements. Nucl. Phys. B 449, 197-228 (1995). arXiv:hep-ph/9409437

54. O. Gedalia, G. Isidori, G. Perez, Combining direct and indirect Kaon CP violation to constrain the warped KK scale. Phys. Lett. B 682, 200-206 (2009). arXiv:0905.3264

55. A.J. Buras, M. Misiak, J. Urban, Two loop QCD anomalous dimensions of flavor changing four quark operators within and beyond the standard model. Nucl. Phys. B 586, 397-426 (2000). arXiv:hep-ph/0005183

56. J. Aebischer, M. Fael, C. Greub, J. Virto, B physics beyond the standard model at one loop: complete renormalization group evolution below the electroweak scale. JHEP 09, 158 (2017). arXiv:1704.06639
57. E.E. Jenkins, A.V. Manohar, P. Stoffer, Low-energy effective field theory below the electroweak scale: anomalous dimensions. JHEP 01, 084 (2018). arXiv:1711.05270

58. J. Aebischer, J. Kumar, D.M. Straub, Wilson: a Python package for the running and matching of Wilson coefficients above and below the electroweak scale. arXiv:1804.05033

59. D.M. Straub et al., Flavio-flavour phenomenology in the standard model and beyond

60. D.M. Straub, Flavio: a Python package for flavour and precision phenomenology in the Standard Model and beyond (2018). arXiv: 1810.08132

61. V. Cirigliano, G. Ecker, H. Neufeld, A. Pich, J. Portoles, Kaon decays in the standard model. Rev. Mod. Phys. 84, 399 (2012). arXiv: 1107.6001

62. G.C. Branco, J.M. Frere, J.M. Gerard, The value of $\epsilon^{\prime} / \epsilon$ in Models Based on $S U(2)_{L} \times S U(2)_{R} \times U(1)$. Nucl. Phys. B 221, 317-330 (1983)

63. J. Aebischer et al., WCxf: an exchange format for Wilson coefficients beyond the standard model. arXiv: 1712.05298

64. J. Aebischer, A. Crivellin, M. Fael, C. Greub, Matching of gauge invariant dimension-six operators for $b \rightarrow s$ and $b \rightarrow c$ transitions. JHEP 05, 037 (2016). arXiv: 1512.02830

65. E.E. Jenkins, A.V. Manohar, P. Stoffer, Low-energy effective field theory below the electroweak scale: operators and matching. JHEP 03, 016 (2018). arXiv: 1709.04486

66. C. Bobeth, U. Haisch, Anomalous triple gauge couplings from $B$-meson and kaon observables. JHEP 09, 018 (2015). arXiv:1503.04829. https://doi.org/10.1007/JHEP09(2015)018

67. E.E. Jenkins, A.V. Manohar, M. Trott, Renormalization group evolution of the standard model dimension six operators I: formalism and lambda dependence. JHEP 10, 087 (2013). arXiv:1308.2627

68. E.E. Jenkins, A.V. Manohar, M. Trott, Renormalization group evolution of the standard model dimension six operators II: Yukawa dependence. JHEP 01, 035 (2014). arXiv: 1310.4838

69. R. Alonso, E.E. Jenkins, A.V. Manohar, M. Trott, Renormalization Group evolution of the standard model dimension six operators III: Gauge coupling dependence and phenomenology. JHEP 04, 159 (2014). arXiv: 1312.2014

70. A. Celis, J. Fuentes-Martin, A. Vicente, J. Virto, Dsix tools: the standard model effective field theory toolkit. Eur. Phys. J. C 77(6), 405 (2017). arXiv: 1704.04504

71. RBC/UKQCD Collaboration, N. Garron, R.J. Hudspith, A.T. Lytle, Neutral Kaon mixing beyond the Standard Model with $N_{f}=2+1$ Chiral Fermions Part 1: bare matrix elements and physical results. JHEP 11, 001 (2016). arXiv:1609.03334

72. RBC, UKQCD Collaboration, P.A. Boyle, N. Garron, R.J. Hudspith, C. Lehner, A.T. Lytle, Neutral kaon mixing beyond the Standard Model with $N_{f}=2+1$ chiral fermions. Part 2: non perturbative renormalisation of the $\Delta F=2$ four-quark operators. JHEP 10, 054 (2017). arXiv: 1708.03552

73. ETM Collaboration, N. Carrasco, P. Dimopoulos, R. Frezzotti, V. Lubicz, G.C. Rossi, S. Simula, C. Tarantino, $\Delta S=2$ and $\Delta C=2$ bag parameters in the standard model and beyond from $N_{f}=2+1+1$ twisted-mass lattice QCD. Phys. Rev. D 92(3), 034516 (2015). arXiv:1505.06639

74. SWME Collaboration, B.J. Choi et al., Kaon BSM B-parameters using improved staggered fermions from $N_{f}=2+1$ unquenched QCD. Phys. Rev. D 93(1), 014511 (2016). arXiv:1509.00592

75. A.J. Buras, J.-M. Gérard, Dual QCD insight into BSM hadronic matrix elements for $K^{0}-\bar{K}^{0}$ Mixing from Lattice QCD. arXiv: 1804.02401

76. J. Brod, M. Gorbahn, Next-to-next-to-leading-order charm-quark contribution to the $\mathrm{CP}$ violation parameter $\varepsilon_{K}$ and $\Delta M_{K}$. Phys. Rev. Lett. 108, 121801 (2012). arXiv:1108.2036 
77. Z. Bai, N.H. Christ, T. Izubuchi, C.T. Sachrajda, A. Soni, J. Yu, $K_{L}-K_{S}$ mass difference from lattice QCD. Phys. Rev. Lett. 113, 112003 (2014). arXiv:1406.0916

78. N.H. Christ, X. Feng, G. Martinelli, C.T. Sachrajda, Effects of finite volume on the $K_{L}-K_{S}$ mass difference. Phys. Rev. D 91(11), 114510 (2015). arXiv: 1504.01170

79. J. Bijnens, J.M. Gérard, G. Klein, The $K_{L}-K_{S}$ mass difference. Phys. Lett. B 257, 191-195 (1991)

80. A.J. Buras, D. Guadagnoli, Correlations among new CP violating effects in $\Delta F=2^{\prime \prime}$ observables. Phys. Rev. D 78, 033005 (2008). arXiv:0805.3887

81. A.J. Buras, D. Guadagnoli, G. Isidori, On $\varepsilon_{K}$ beyond lowest order in the operator product expansion. Phys. Lett. B 688, 309-313 (2010). arXiv:1002.3612

82. M. Bobrowski, A. Lenz, J. Riedl, J. Rohrwild, How large can the SM contribution to CP violation in $D^{0}-\bar{D}^{0}$ mixing be? JHEP 03, 009 (2010). arXiv:1002.4794

83. HFLAV Collaboration, Y. Amhis et al., Averages of $b$-hadron, $c$ hadron, and $\tau$-lepton properties as of summer 2016. Eur. Phys. J. C 77(12), 895 (2017). arXiv:1612.07233

84. K. Blum, Y. Grossman, Y. Nir, G. Perez, Combining $K^{0}-\bar{K}^{0}$ mixing and $D^{0}-\bar{D}^{0}$ mixing to constrain the flavor structure of new physics. Phys. Rev. Lett. 102, 211802 (2009). arXiv:0903.2118

85. V. Cirigliano, W. Dekens, J. de Vries, E. Mereghetti, An $\epsilon^{\prime}$ improvement from right-handed currents. Phys. Lett. B 767, 1-9 (2017). arXiv: 1612.03914

86. PNDME Collaboration, T. Bhattacharya, V. Cirigliano, S. Cohen, R. Gupta, A. Joseph, H.-W. Lin, B. Yoon, Iso-vector and Iso-scalar Tensor Charges of the Nucleon from Lattice QCD. Phys. Rev. D 92(9), 094511 (2015). arXiv:1506.06411

87. C. Alexandrou et al., Nucleon scalar and tensor charges using lattice QCD simulations at the physical value of the pion mass. Phys. Rev. D 95(11), 114514 (2017). arXiv:1703.08788. [Erratum: Phys. Rev.D96,no.9,099906(2017)]

88. JLQCD Collaboration, N. Yamanaka, S. Hashimoto, T. Kaneko, H. Ohki, Nucleon charges with dynamical overlap fermions. arXiv: 1805.10507

89. R. Gupta, B. Yoon, T. Bhattacharya, V. Cirigliano, Y.-C. Jang, H.-W. Lin, Flavor diagonal tensor charges of the nucleon from 2+1+1 flavor lattice QCD. arXiv:1808.07597

90. M. Pospelov, A. Ritz, Neutron EDM from electric and chromoelectric dipole moments of quarks. Phys. Rev. D 63, 073015 (2001). arXiv:hep-ph/0010037

91. K. Fuyuto, J. Hisano, N. Nagata, K. Tsumura, QCD Corrections to quark (Chromo)-electric dipole moments in high-scale supersymmetry. JHEP 12, 010 (2013). arXiv:1308.6493

92. J.M. Pendlebury et al., Revised experimental upper limit on the electric dipole moment of the neutron. Phys. Rev. D D92(9), 092003 (2015). arXiv:1509.04411

93. N. Yamanaka, B.K. Sahoo, N. Yoshinaga, T. Sato, K. Asahi, B.P. Das, Probing exotic phenomena at the interface of nuclear and particle physics with the electric dipole moments of diamagnetic atoms: a unique window to hadronic and semi-leptonic $\mathrm{CP}$ violation. Eur. Phys. J. A 53(3), 54 (2017). arXiv: 1703.01570
94. V. Cirigliano, S. Gardner, B. Holstein, Beta decays and nonstandard interactions in the LHC Era. Prog. Part. Nucl. Phys. 71, 93-118 (2013). arXiv:1303.6953

95. M. González-Alonso, J Martin Camalich, Global effective-fieldtheory analysis of new-physics effects in (semi)leptonic kaon decays. JHEP 12, 052 (2016). arXiv: 1605.07114

96. M. González-Alonso, O. Naviliat-Cuncic, N. Severijns, New physics searches in nuclear and neutron $\beta$ decay. arXiv: 1803.08732

97. K.O.T.O. Collaboration, K. Shiomi, Search for the rare decay $K_{L} \rightarrow \pi^{0} v \bar{v}$. Talk at ICHEP 2018, 7 (2018)

98. G. Isidori, J.F. Kamenik, Z. Ligeti, G. Perez, Implications of the LHCb evidence for charm CP violation. Phys. Lett. B 711, 46-51 (2012). arXiv:1111.4987

99. J. de Blas, J.C. Criado, M. Perez-Victoria, J. Santiago, Effective description of general extensions of the standard model: the complete tree-level dictionary. JHEP 03, 109 (2018). arXiv: 1711.10391

100. J.M. Arnold, B. Fornal, M.B. Wise, Simplified models with baryon number violation but no proton decay. Phys. Rev. D 87, 075004 (2013). arXiv: 1212.4556

101. N. Assad, B. Fornal, B. Grinstein, Baryon number and Lepton Universality violation in leptoquark and diquark models. Phys. Lett. B 777, 324-331 (2018). arXiv:1708.06350

102. A.J. Buras, F. De Fazio, J. Girrbach, $\Delta I=1 / 2$ rule, $\varepsilon^{\prime} / \varepsilon$ and $K \rightarrow \pi \nu \bar{v}$ in $Z^{\prime}(Z)$ and $G^{\prime}$ models with FCNC quark couplings. Eur. Phys. J. C 74(7), 2950 (2014). arXiv: 1404.3824

103. S. Alioli, V. Cirigliano, W. Dekens, J. de Vries, E. Mereghetti, Right-handed charged currents in the era of the Large Hadron collider. JHEP 05, 086 (2017). arXiv:1703.04751

104. K. Agashe, A. Azatov, L. Zhu, Flavor violation tests of warped/composite SM in the two-site approach. Phys. Rev. D 79, 056006 (2009). arXiv:0810.1016

105. C. Delaunay, J.F. Kamenik, G. Perez, L. Randall, Charming CP violation and dipole operators from RS flavor anarchy. JHEP 01, 027 (2013). arXiv: 1207.0474

106. N. Vignaroli, $\Delta \mathrm{F}=1$ constraints on composite Higgs models with LR parity. Phys. Rev. D 86, 115011 (2012). arXiv:1204.0478

107. M. König, M. Neubert, D.M. Straub, Dipole operator constraints on composite Higgs models. Eur. Phys. J. C 74(7), 2945 (2014). arXiv:1403.2756]

108. WCxf Collaboration, flavio WCxf basis file. https://wexf.github. io/assets/pdf/WET.flavio.pdf

109. A.L. Kagan, Right-handed currents, CP violation, and $B \rightarrow V V$. arXiv:hep-ph/0407076 